Chapman University

Chapman University Digital Commons

Education (PhD) Dissertations

Dissertations and Theses

Summer 8-2019

\title{
Minding the Gap: A Grounded Theory Exploration of Transition from Secondary School to Community College for Students Identified with Autism
}

Douglas Highlen

Chapman University, highl104@mail.chapman.edu

Follow this and additional works at: https://digitalcommons.chapman.edu/education_dissertations

Part of the Disability and Equity in Education Commons, Higher Education and Teaching Commons, Secondary Education Commons, and the Secondary Education and Teaching Commons

\section{Recommended Citation}

Highlen, D. (2019). Minding the gap: A grounded theory exploration of transition from secondary school to community college for students identified with autism [Doctoral dissertation, Chapman University]. Chapman University Digital Commons. https://doi.org/10.36837/chapman.000084

This Dissertation is brought to you for free and open access by the Dissertations and Theses at Chapman University Digital Commons. It has been accepted for inclusion in Education (PhD) Dissertations by an authorized administrator of Chapman University Digital Commons. For more information, please contact laughtin@chapman.edu. 
Minding the Gap: A Grounded Theory Exploration of Transition from Secondary School to Community College for Students Identified with Autism

\author{
A Dissertation by \\ Douglas L. Highlen \\ Chapman University \\ Orange, CA \\ Attallah College of Educational Studies \\ Submitted in partial fulfillment of the requirements for the degree of \\ Doctor of Philosophy in Education \\ August 2019 \\ Committee in charge: \\ Scot Danforth, Ph.D., Chair \\ Meghan Cosier, Ph.D. \\ Phil Ferguson, Ph.D.
}


The dissertation of Douglas L. Highlen is approved.

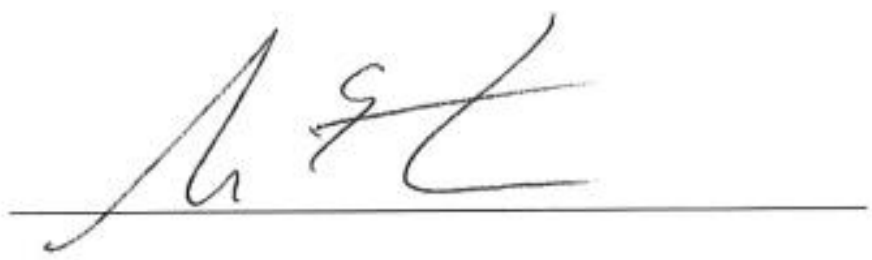

Scot Danforth, Ph.D., Chair
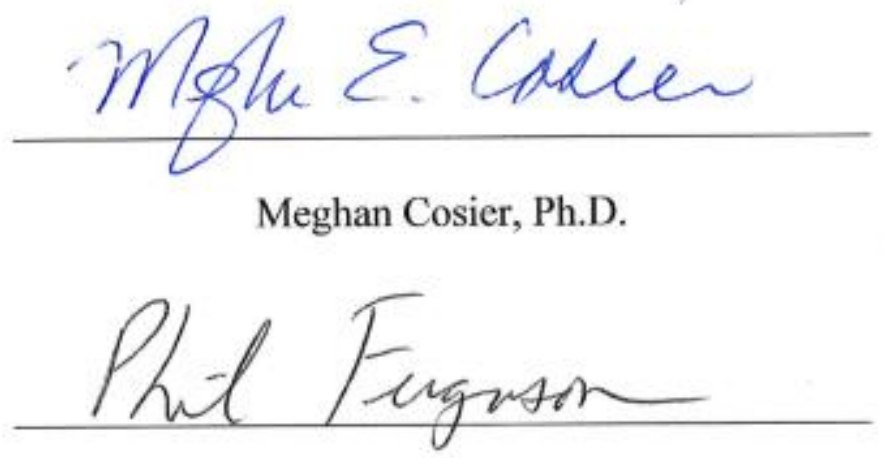

Phil Ferguson, Ph.D.

June 2019 
Minding the Gap: A Grounded Theory Exploration of Transition from Secondary School to Community College for Students Identified with Autism

Copyright $\odot 2019$

by Douglas L. Highlen 


\section{DEDICATION}

\section{To Frank and Colleen Highlen (my parents)}

For believing in the transformative power of education.

To Arlene Highlen (my beloved wife)

For supporting and encouraging me throughout this process.

Thank you for your love and support. 


\section{ACKNOWLEDGEMENTS}

I would like to express my sincere gratitude and appreciation to my dissertation committee. Only through their guidance and support could I have possibly navigated this process successfully.

To Dr. Scot Danforth, thank you recognizing early in my doctoral journey that I was capable of much more than I believed I was. Thank you for agreeing to serve as my committee chair and provide me with the ongoing encouragement necessary to take this old secondary school teacher and remake him into an academician. Thank you for everything. John Dewey said, "Failure is instructive". During the last four years I have benefitted from considerable instruction.

To Dr. Phil Ferguson, thank you for agreeing so serve on my committee. I entered the Chapman University doctoral program with one wish from my wife, Arlene (MA, 2013), "I hope you get to take a class from Phil Ferguson". I did get to take a class from you during the fall of 2015, and greatly appreciate having had the experience. Your willingness to subsequently serve on my committee means the world to me.

To Dr. Meghan Cosier, thank you for challenging me to think more deeply and completely throughout the dissertation process. Thank you for helping me to expand the boundaries of my thinking to ensure rigorous qualitative research.

To Dr. Dawn Hunter, thank you for taking a chance with me. As director of the Ph.D. program, you gave me the opportunity to enter the doctoral program as a nontraditional student. My age and the many years I had spent away from university made for a rather steep learning curve, but I have worked tirelessly to confirm your confidence in me, and I believe this dissertation provides that confirmation. 
To Dr. Whitney McIntyre-Miller, who introduced me to Grounded Theory and provided valuable ongoing support for my data analysis. I am grateful for your willingness to support me in the development of emergent theory. I could not have done this without you.

I could not have weathered my Ph.D. journey without the love and support of my family. My parents, though long ago passed, still hold a privileged position in this odyssey. Though coming from humble midwestern stock, and never having the opportunity to pursue a postsecondary education, they instilled in me the values of hard work and education, and for that I am eternally grateful. I want to thank my wife, Arlene, for her love, support, and editing acumen; and my children Colleen, Alison, and Christopher for their encouragement and support throughout this process.

I would like to acknowledge the Attallah College of Educational Studies at Chapman University for an outstanding Ph.D. program and all the staff members for their support. Moreover, a special thank you to Dr. Randy Busse, Dr. Don Cardinal, Dr. Kris De Pedro, Dr. Michael Hass, Dr. Kelly Kennedy, Dr. Whitney McIntyre-Miller, and Dr. Lilia Monzó for helping me expand my knowledge and pushing me to think in new ways; I have come to understand that there are multitudes of knowing, and in reference to a discussion held early in the doctoral program, "a wall may not always be a wall." I wish to acknowledge the support received from my 2015 Disability Studies Cohort: Lisa, Krista, and Lara define for me dedication, commitment and scholarly rigor. Throughout the process, I could take from their example the strength needed to push forward. I will miss you all. 
Last but certainly not least, I wish to thank the 26 individual participants engaged in my study. To the students, I encourage you to push beyond whatever roadblocks may appear along the way to persist in your academic goals. To the parents, I empathize with the challenges that you may face, and ask that you redouble your efforts on behalf of your children. To the practitioners, I understand the convoluted, byzantine architecture in which you service your clients. I applaud you and encourage you to, in spite of it all, continue to find ways to put the needs of the students first. Thank you for allowing me to take this journey of transition with you. Thank you for trusting me with your thoughts and feelings and for believing that I could do justice to your experiences; for that, I will be forever grateful. 


\begin{abstract}
Minding the Gap: A Grounded Theory Exploration of Transition from Secondary School to Community College for Students Identified with Autism

by Douglas L. Highlen
\end{abstract}

This qualitative research study aims to facilitate a better understanding of the process of transition from secondary school to community college for students identified with autism. Previous research indicates that though most students identified with autism report that they have strong intentions of pursuing a postsecondary education, comparatively few achieve this goal. Prior research on transition has focused on strategies utilized by secondary schools to facilitate success for students identified with autism or postsecondary institutional approaches utilized once a student has arrived at the school. This bifurcated approach has resulted in a gap in the research in that virtually no studies have considered transition from a unified perspective, nor has any serious attention been given to the summer "gap" that exists between secondary school and community college. Through a grounded theory exploration, the experiences and perspectives of college-bound students identified with autism, their parents/caregivers, high school and community college faculty and other stakeholders resulted in the emergence of foundational elements central to understanding what critical factors influence these students and how these students and stakeholders experience this transition. Furthermore, these foundational elements provide, within the confines of this study, a preliminary model of transition for students identified with autism. 


\section{TABLE OF CONTENTS}

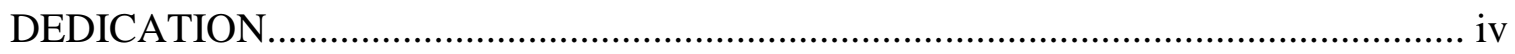

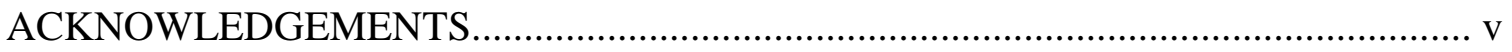

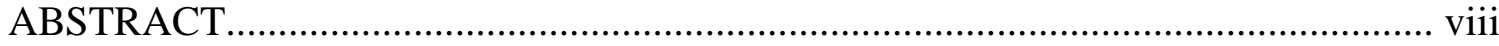

LIST OF FIGURES .............................................................................. xii

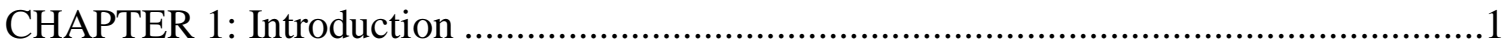

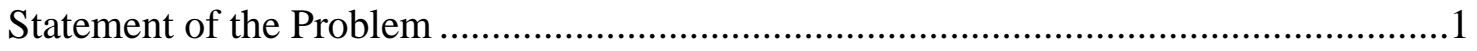

Research Questions ............................................................................................4

Significance of the Problem .................................................................................4

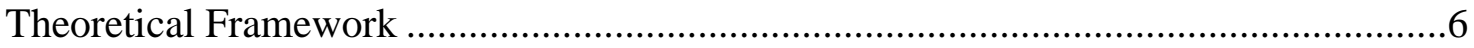

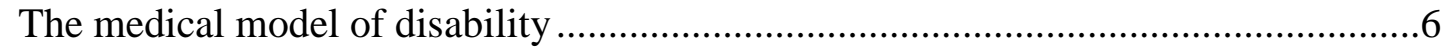

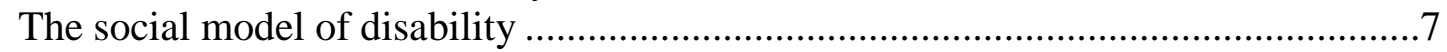

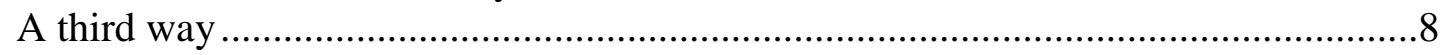

Theoretical models of disability and transition to college....................................11

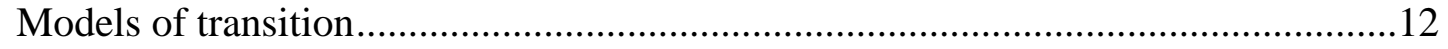

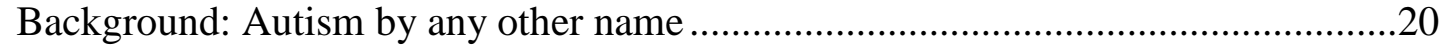

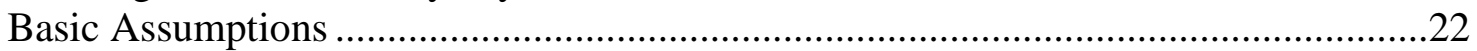

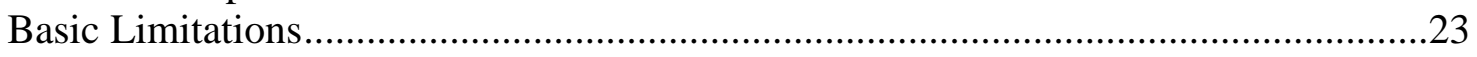

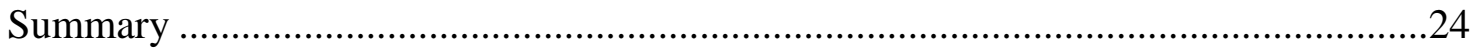

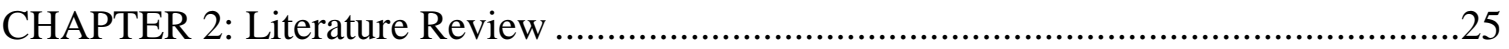

Why Transition to College? .............................................................................27

Factors that Promote Transition from Secondary School for Students Identified with

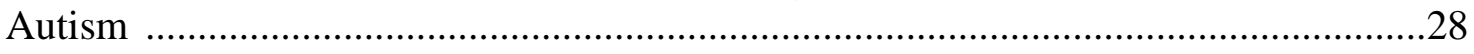

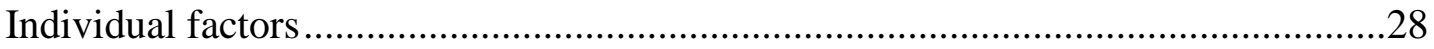

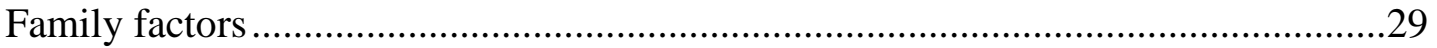

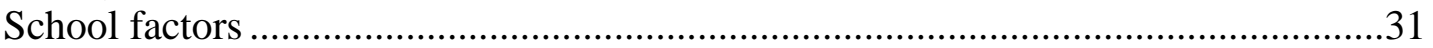

Factors that Promote Success for Students Identified with Autism as they Transition

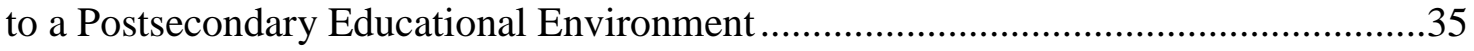

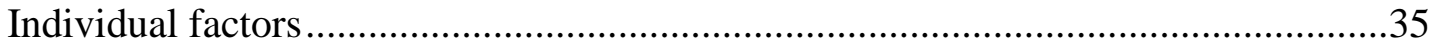

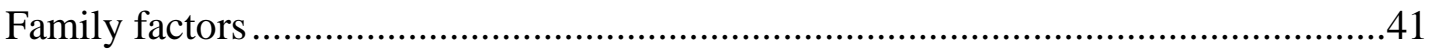

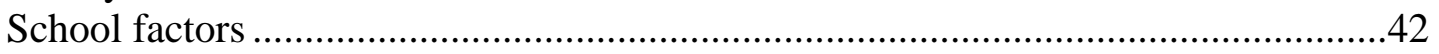

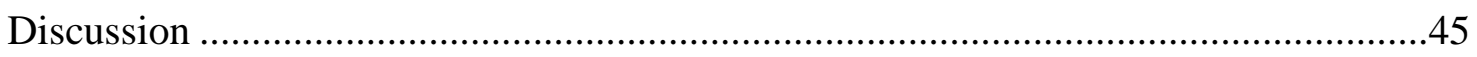

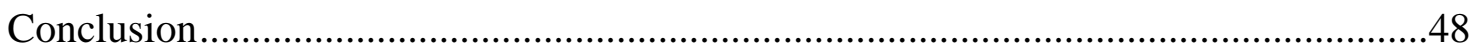

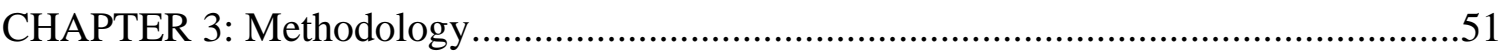

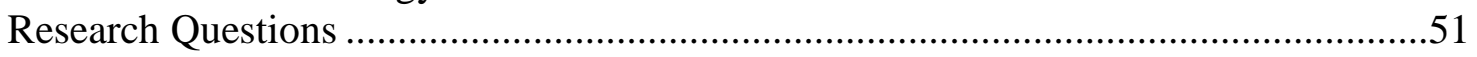

Reasons for Utilizing a Qualitative Approach ...................................................52

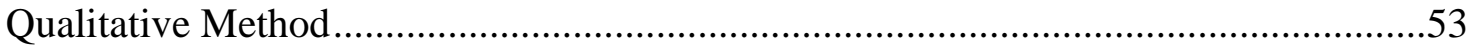

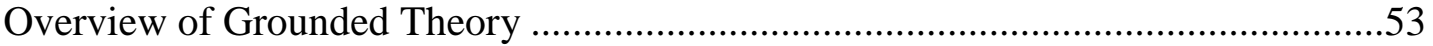

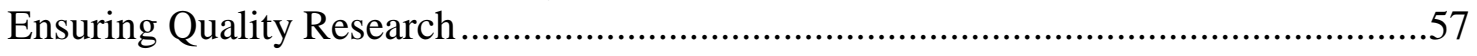




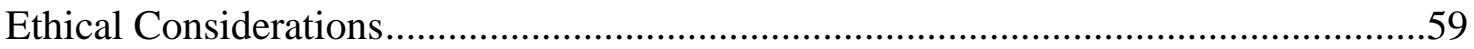

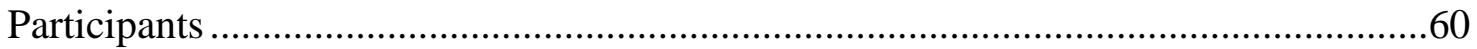

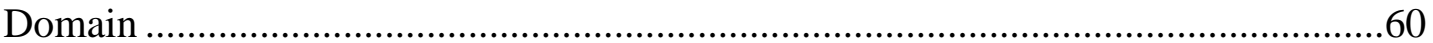

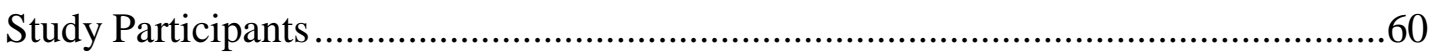

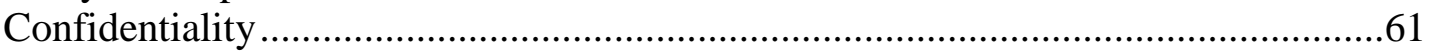

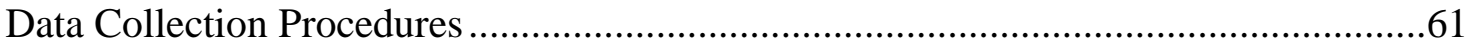

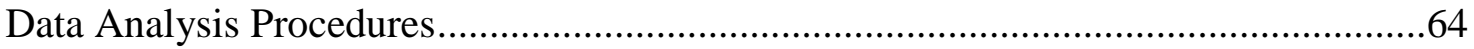

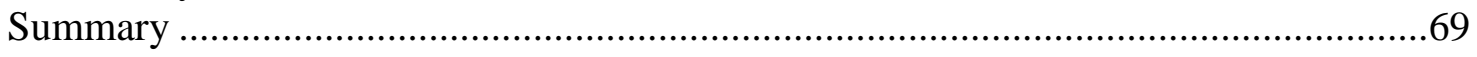

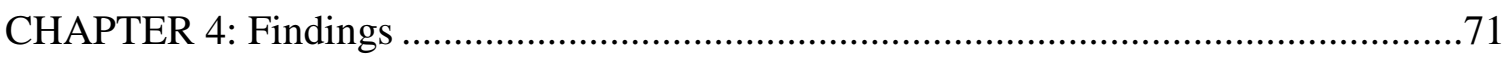

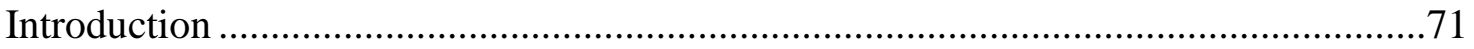

Secondary School Architecture and Culture …………...........................................

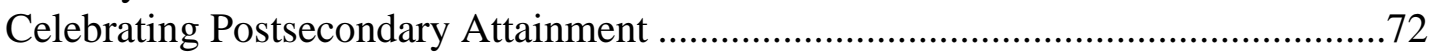

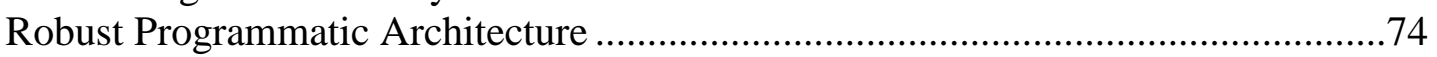

A Tradition of Focus on Four-Year Institutions......................................................76

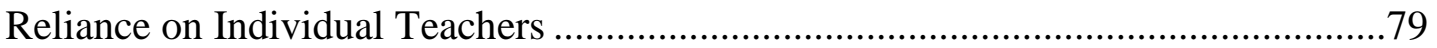

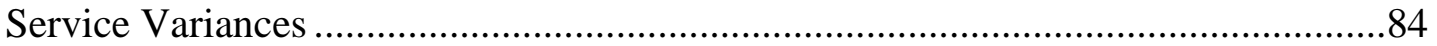

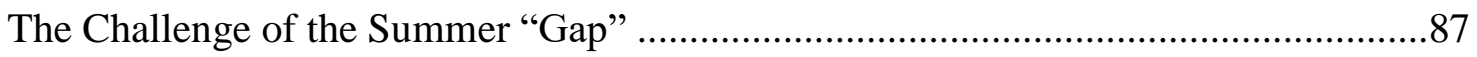

"we wave goodbye and they are magically cured"- The End of Secondary School..87

"playing Xbox with my friends" - The Unstructured Nature of the Gap ....................88

Community College Architecture and Culture.............................................................89

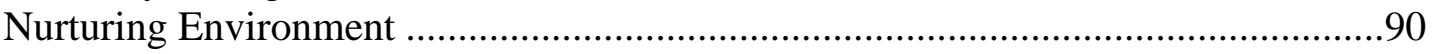

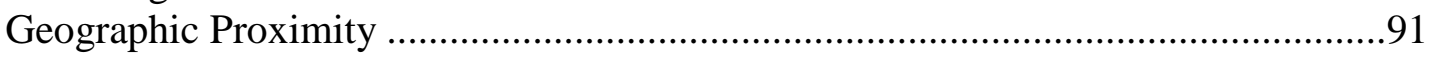

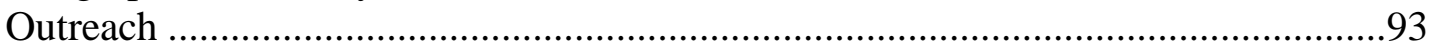

Reliance on Grant Funding..............................................................................99

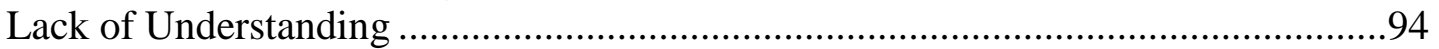

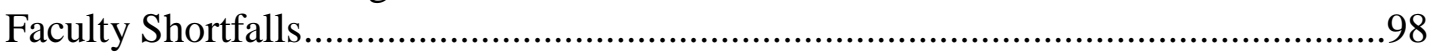

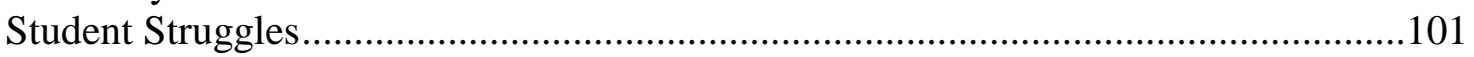

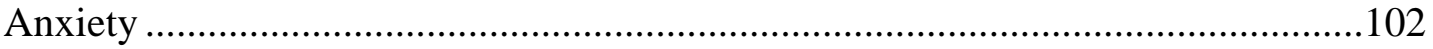

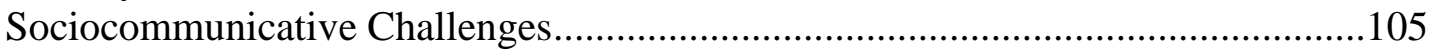

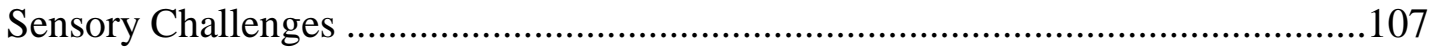

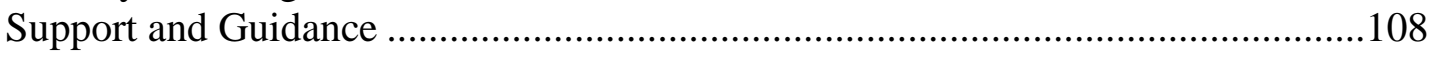

The Essential Nature of Personal Support From a "Familiar" ......................................112

"they basically forced me to go" - Parents .............................................................113

"I spent a lot of time with my aunt" - Extended Family..........................................117

"One of my friends named Luis used to come here" - Peers ....................................118

"you know, probably a buddy system" - Mentors .................................................119

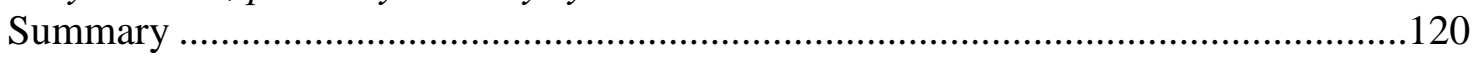

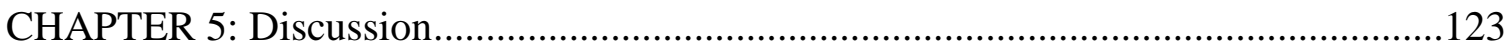

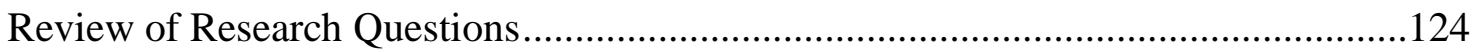

Secondary School Architecture and Culture …………............................................124

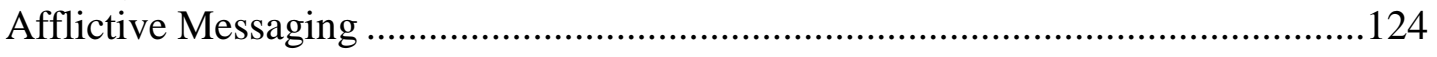

Shortfalls between programmatic transition architecture and practical implementation .............................................................................................129

The Medical Model and Service Variances.............................................................136 
Foundational Element Summary ……………………........................................139

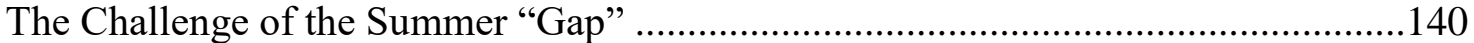

"graduation can be abrupt" - The End of Routine...................................................140

"I've been doing a lot of sleeping" - The Unstructured Nature of the "Gap" .........142

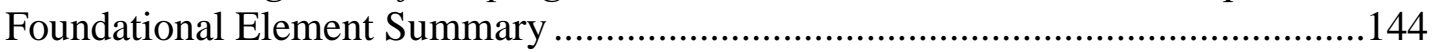

Community College Architecture and Culture..........................................................144

"I honestly feel very supported at school" - A Nurturing Environment ...................145

"everyone who works there talks down to you" - Lack of DSPS Understanding ....146

"We do not run into very many difficulties with faculty" - Faculty Shortfalls..........148

Foundational Element Summary ……………………….....................................150

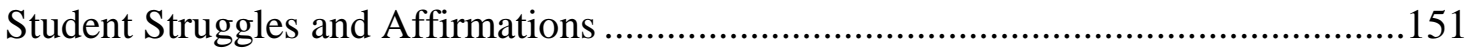

Foundational Element Summary ……………………....................................154

The Essential Nature of Personal Support...........................................................154

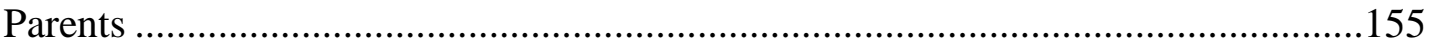

Extended Family and Peers ............................................................................156

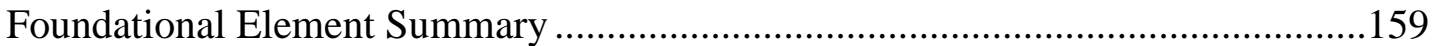

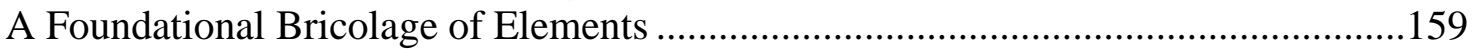

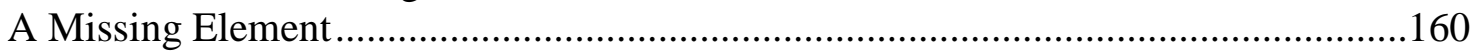

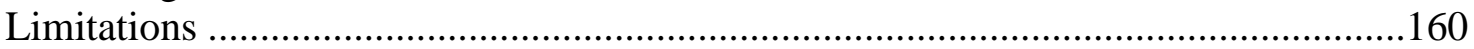

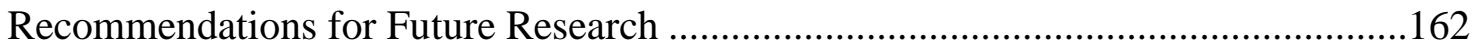

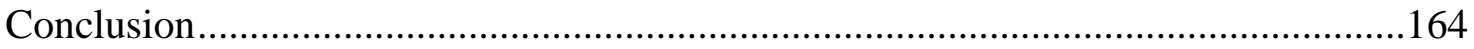

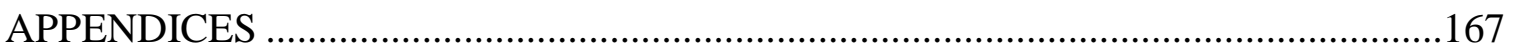

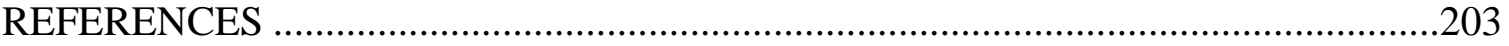




\section{LIST OF FIGURES}

Figure 1. Graphic representation of Schlossberg's (2012) Transition Theory .................14

Figure 2. Graphic representation of Bean and Metzner's (1985) Attrition Model ...........19 


\section{Chapter I: Introduction}

This qualitative research study explored the experiences and perspectives of college-bound students identified with autism, their parents/caregivers, high school and community college faculty and other stakeholders as these students transitioned from secondary school to community college. A consideration of the central problem with research questions, the significance of the problem, the overarching theories and models influencing the course of the study, a brief historical sketch of the changing verbiage relative to autism, as well as basic assumptions and limitations will be discussed in this chapter. A review of the relevant literature pertaining to factors influencing successful transition for students identified with autism is presented in chapter two. The methodology embraced, practical methods adopted and a description of the format of the study are provided in chapter three. The results of the study are presented in chapter four. Finally, a discussion of the findings, implications for future research, study limitations and recommendations are addressed in chapter five.

\section{Statement of the Problem}

Nearly 3.1 million students graduated from high school in the United States in 2016 and 2.2 million of those (69.7\%) enrolled in college (Bureau of Labor Statistics, 2017). Whereas graduation was once a conclusion of formal education for most and the onset of career work, it is now often a momentary time of rejoicing before entrance into a postsecondary educational institution. This period of transition can be dramatic; a student leaves the structured setting of school and the omnipresent supervision of parents for an environment of independence, experience and options. 
Among college enrollees is a population of students identified with autism (Mason, Rispoli, Ganz, Boles, \& Orr, 2012). While the exact numbers are uncertain, the percentages paint a picture of significant concern relative to their success; data analysis of the National Longitudinal Transition Study 2 (NLTS2) indicated that over $84 \%$ of students identified with autism reported while in secondary school that they would "definitely" or "probably" get some form of postsecondary education (Wagner, Newman, Cameto, Levine, \& Marder, 2007), but nearly $68 \%$ of these students did not apply for admittance to postsecondary institutions, were not offered admittance to higher education, or failed to persist once they were admitted (Glennon 2001; Wei et al. 2012). Acknowledging that not all students expressing an interest in college will ultimately attend, this discrepancy between intent and persistence still represented a significant gap that is worthy of consideration. What is it about the secondary school setting that results in most of these students articulating that they will attend a postsecondary institution? What happened after the articulation of these beliefs that resulted in the student either never attending or failing to persist in a postsecondary institution? What can be done to foster transitional success?

Community college is one of several available postsecondary educational options. While the numbers of students identified with autism attending community college is a matter of some debate, a 2011 U.S. Department of Education report stated that $32.6 \%$ of students who were identified as having autism had attended community college during the six years following their secondary education (Sanford et al., 2011). Other studies have suggested that attendance by students with autism spectrum disorder (ASD) at a community college is as high as $81.33 \%$ (Wei et al., 2014). Regardless, it is likely that 
attendance will increase over time, necessitating an increased imperative for understanding individuals with autism (Vanbergeijk, Klin, \& Volkmar, 2008). This likely increase in attendance led the researcher to focus on community colleges as the sole postsecondary institution for this study.

Beyond the imperative illustrated by the discrepancy between the students' stated intents and their persistence, there existed a gap in the research as well. The process of transition from secondary to a postsecondary educational setting for students identified with autism has been traditionally understudied, with the primary focus of research into autism conducted at the early childhood and elementary level. This was likely the result of the capacity for early diagnosis (Shattuck et al, 2012) and the reported benefits of swift and early intervention (Ben Itzchak \& Zachor, 2011; Lazaratou, Economou, \& Dikeos, 2017). This does not mitigate the need for study of transition for emerging adults. Indeed, the efforts exerted towards childhood research and the resultant treatments likely have increased the numbers of individuals identified with autism who find themselves able to pursue a college trajectory.

While there has been recent studies conducted that explore the experiences of students with autism during transition from a secondary to a postsecondary setting (Jones, 2016; Poleyeff, 2015; Shepherd, 2015), their parents (Ebel, 2015), or directors of offices of disability/accessibility at the postsecondary level (Sutton-Young, 2015), as yet no effort had been made to explore the experiences of these students and key participants from a unified perspective. By exploring these participants as discrete entities, researchers missed the opportunity to discover disparities of experience which may hold the key to understanding why so many students intend to succeed in college but realize 
much humbler results. Furthermore, the study of transition as the discrete experiences of delineated groups has had the unintended effect of creating a barrier between secondary research and postsecondary research. Transition should be a near seamless experience, marked by a developing awareness and readiness for change and a gradual introduction of the new reality. Continuing to explore transition as discrete elements fractures the experience into easily studied but ultimately less helpful components. A unified understanding of transition from high school to community college for students identified with autism was called for; this dissertation initiated the efforts towards such an understanding.

\section{Research Questions:}

Two research questions guided this study:

1. What are the critical factors that influence college trajectory students identified with autism as they transition from a secondary school to a community college?

2. How do students with autism and other stakeholders experience this transition?

\section{Significance of the Problem}

Previous research into the transition of students identified with autism from secondary school to a postsecondary educational setting is scarce, more so when considering community colleges and essentially nonexistent when told from a unified perspective. One collective case study of transition was conducted within a rural school district in Alabama, but only involved secondary school students, parents and personnel, and focused on the transition planning component of the Individual Education Plan (IEP) (DeLeo, 2017). This paucity of research represented a significant gap within the academic literature. 
Beyond the presence of a gap in research, there is a legitimate and growing need for understanding and assisting in the transition of students identified with autism from high school to community college. In the fall of 2017 , approximately 50.7 million children were attending elementary and secondary schools in the United States (Institute of Education Sciences, 2018). How many of these students are or will be identified with autism and wish to pursue a community college education is uncertain. Current literature reports that nearly 50,000 students with ASD turn eighteen each year in the United States (Shattuck et al, 2012). If this number is to be accepted, one could extrapolate that as many as 42,000 of these students report an intent to pursue a postsecondary education but only 16,000 apply, enroll and persist (Glennon 2001; Wagner et al., 2007; Wei et al. 2012). This represents a significant gap between student intentions and results, and one worthy of scholarly research.

The yearly prospect of tens of thousands of students with autism either failing to attempt postsecondary education or desisting once admitted to college constitutes a significant cost to these individuals in terms of lost educational, social and financial opportunity as well as a loss to society in terms of human capital. Frankly, it represented a loss of individual and social potential too significant to passively condone. As more and more students identified with autism are choosing to attend postsecondary schools (Mason et al., 2012), it becomes incumbent upon researchers to better understand the transition process and identify the causes of the discrepancy between the student's initial intent and their ultimate performance. Factors that promoted or inhibited a successful transition from secondary school to a postsecondary environment must be understood and 
mechanisms must be devised for promoting success for these students within an inclusive collegiate setting.

The paucity of research into transition for students with autism from secondary to postsecondary school, and the absence of research exploring this transition from a unified perspective, indicated that this study had the potential to be an important addition to the small body of research that already exists addressing students with autism and their transition to college.

\section{Theoretical Framework}

\section{The medical model of disability}

The scant research into transition from high school to college was largely comprised of work conducted and/or presented from a medical model perspective. This was not altogether surprising. The medical model was an outgrowth of the biomedical approach to medicine, which has been practiced since the mid-nineteenth century (Meng, 2014). It defines society as either "disabled" or "healthy" and places the source of the disability within the individual. Through this approach decisions can be made as to the development of treatments that mitigate or hopefully cure the individual (Areheart, 2008). Applicable law and the Diagnostic and Statistical Manual of Mental Disorders 5th ed. (DSM-5) tend to lean heavily on, if not altogether embrace, the medical model. This impact of the DSM-5 is especially insidious; by promulgating and holding hegemony over the terms used to describe the conditions, the APA's actions tend to control the discourse. As a result, researchers who wish to ensure funding and educational institutions that wish to ensure compliance may find it beneficial to favor a medical approach, as evidenced by terms used throughout the literature such as "high-functioning 
autism", "Asperger's syndrome” or "autism spectrum disorder". The use of these terms normalizes autism as a deficiency; the mindset they engender promotes a medicalization of autism which "others" the disabled. This undermines the individual, as it measures them against a theoretical "norm" and finds them "deficient" (Shyman, 2016). While it might be understandable to establish 20/20 vision as "normal" and prescribe eyewear to address variances in eyesight, the diversity of human cognitive processes does not lend itself to a similar, norm-based mindset.

\section{The social model of disability}

The social model has its origins in the independent living movement of Ed Roberts and others at Cal Berkeley in the 1960s (Brown, 2017); was more formally developed by the Union of the Physically Impaired Against Segregation (UPIAS) of the 1970s (UPIAS, 1976), and was defined by Oliver (1983) as a model of disability in contrast to the medical model. Whereas the medical model views a disability as an intrinsic individual condition to be identified, treated, and mitigated or cured, the social model places responsibility for disablement of individuals squarely upon society. UPIAS aptly defined disability in terms of the restriction and marginalization placed on individuals by society:

The disadvantage of restriction of activity caused by a contemporary social organisation (sic) which takes no or little account of people who have [impairments] and thus excludes them from the mainstream of social activities (UPIAS, 1976, p 14)

Whereas the thrust of the medical approach to disability is to change the individual to a condition more closely mimicking "the norm", the thrust of the social 
model is to change society. UPIAS was the first to delineate between impairment and disability (Shepherd, 2015). An individual might exhibit an impairment, such as a restriction or malfunction of a limb (Goodley, 2001), but "disability" is a restriction or disadvantage experienced by an individual because of social structures that do not account for their impairment and therefore exclude them from involvement in society (Goodley, 2001). This distinction is critical in that it articulates that it is not one's impairment that disables, but rather society that disables by its structures and organizations. From this perspective, disability can be substantially alleviated by focusing on the creation of inclusive social mechanisms and universal designs (Campbell \& Oliver, 1996).

This researcher contends that the efforts of UPIAS and those who have followed were not a call for all medical interventions to be abandoned, nor was it a declaration of abrogation on the part of the individual with impairment, but rather it was a petition for greater emphasis on dismantling the disabling barriers of society. To this end, the writings of the late Australian neurodiversity advocate Vale Polly Samuel (aka "Donna Williams") bear consideration. (Williams, 2014).

\section{A third way...}

According to Williams (2014), the medical model places "blame" on the individual and ascribes as "tragedy" their impairment, while the social model places "blame" on society; she eschewed both of these perspectives as incomplete and often overutilized in favor of what she termed an empowerment model of disability, where one is fully informed and can decide for oneself what aspects of one's impairment are best viewed as strengths that need advocacy and appreciation and which aspects are better 
addressed through adaptations or treatment. The only goal of the empowerment model of disability is that of empowering the individual.

Implicit in the empowerment model is the acknowledgement that the individual with autism can assess their strengths and challenges for themselves to make decisions relative to advocacy and/or treatment; to assume otherwise would be to perpetuate a caretaker mentality. As it is the right of the individual to make such determinations, what should be considered a strength and what should be addressed through accommodations would be individually determined.

The empowerment model of disability has yet to develop traction as an overarching theory driving disability studies in academia. This researcher viewed empowerment less as a new model of disability and more as a strategy for utilizing preexisting models. For the college bound student with autism, there can be situations where such an approach might be both pragmatic and effective. For example, the empowerment model is of use when delineating between that which is external to the individual and that which is internally driven.

Research has acknowledged the importance of self-advocacy skills in ensuring that students with autism receive the accommodations needed (Adreon \& Durocher, 2007; Daly-Cano, Vaccaro \& Newman, 2015; Kimball, Moore, Vaccaro, Troiano \& Newman, 2016; Roberts, 2010), yet obtaining those accommodations requires that the individual identify themselves as somehow "other". Sayman (2015) shared this challenge by relating the experience of Jillian (a pseudonym). Even after obtaining employment and gaining confidence, she still needed to improve her self-advocacy skills: 
"I was braver in my classes, but I wasn't as brave to ask for help. I should have asked for more help, especially when I started having trouble. I thought, 'well, I think I'll be okay'... and I wasn't.” (Sayman, 2015, p 88)

As Jillian illustrated, self-advocacy necessitates an externalization of one's autism; inclusive designs and universal approaches to instruction would minimize the need for such externalizations.

Conversely, ensuring that one understands and is understood within a social setting necessitates a degree of cognitive analysis, an inherently internal process. Active strategies for addressing this process (Gallagher, 2004) would seem to mitigate these challenges. Zahavi and Parnas (2003) cited the example of Temple Grandin, who uses active strategies such as reading about people and observing them to explain and predict their actions; she uses this process to decode intentions and states of mind, as an internal accommodation (Zahavi and Parnas, 2003). As this "accommodation" is internally driven, its implementation is dependent on the actions of the individual identified with autism, not society.

The empowerment model offers an opportunity for an individual to leverage both the medical and social model for the potential utility they provide without the necessity for the privileging of one model and the degradation of the other.

As an individual might embrace the empowerment approach to allow for selecting individual action or social accommodation as the mechanism that best meets their individual variances, so this researcher embraced the empowerment approach as a means of privileging both medical model data and social model perspective. Virtually all of the professionals interviewed within the study came from a medical model background and 
spoke from a medical model perspective. Parents likewise often expressed their views through the lens of the medical professionals on whom they'd relied for guidance with their children. The empowerment approach allowed this researcher to respect and privilege their knowledge and perspectives, while concurrently using his own social model perspectives in the analysis and formulation of responses to emergent themes.

\section{Theoretical models of disability and transition to college}

Within this study the author embraced the social model of disability for its usefulness in facilitating a non-stigmatizing social mechanism for effective transition from a secondary to a post-secondary educational setting for students identified with autism. It has been well established that students identified with autism experience myriad social challenges during their transition to a post-secondary institution (Adreon \& Durocher, 2007; Gelbar, Smith, \& Reichow, 2014; Sayman, 2015; White, Ollendick, \& Bray, 2011). The medical model approach resulted in labeling these students as disabled or deficient; as such, it likely exacerbated their internal struggles, potentially resulting in additional external social challenges and possible failure to persist.

In stark contrast, utilizing the social model of disability as a lens offered a view of students identified with autism on a college path as individuals whose diverse ways of processing data provided strengths within the academic setting. Additionally, the social model encourages society to develop structures that are inclusive and universal, thereby mitigating stigma. For these reasons, the social model was the primary disability model utilized within this dissertation; research was conducted, and data analyzed from the perspective of the need for universal and inclusive approaches to embrace the diversity that students identified with autism brought to the transition experience. 


\section{Models of transition}

The human experience is a series of transitions; some are gradual and seamless, and others are abrupt and precarious. Whether a student identified with autism experiences the former or latter in their transition from secondary school to a postsecondary academic environment is dependent upon the actions and reactions of the student, parents and other stakeholders in the process. Awareness of relevant theoretical models pertinent to transition informed the research without constraining the findings to any specific preestablished model. The researcher leveraged preexisting models of transition as the existent terrain but constructed the architecture of the unifying model from the data itself. This is further explained within the discussion of methodology.

\section{Morrison and Clark's Loss Aversion Theory.}

The initial stage of transition is the inevitable movement out of secondary school; a sort of "migration" from the known to the relative unknown. Morrison and Clark (2016) addressed this concern, though from an unexpected perspective. As geographers with a focus in demographics, these researchers typically addressed considerations of population movement: the whys and wherefores of migration. In doing so, they acknowledged that moving was simply an adjustment toward a desired state of stability; existing dwellings and possessions were ascribed a stability value beyond mere purchase price, and that value had the likely effect of delaying migration. Those who ascribed little value to their current environment were more likely to readily migrate.

The migration from secondary school to a postsecondary educational setting can be challenging and especially problematic for many students identified with autism. The rules that neurotypical students often find constraining in secondary school may well 
provide structure and security for many students with autism. Likewise, the transition to a collegiate setting can provide the freedom that many students desire but cause uncertainty for students identified with autism. The "desired state of stability" referenced by Morrison and Clark might be better described as a "desired state of independence" by most graduating students but as an "undesirable state of flux" by those with autism.

Research by Browning, Osborne and Reed (2009) indicated that as students with ASD approached the conclusion of their secondary school experience, they perceived themselves to be less able to deal with stress and were unable to identify the reasons why (Browning et al., 2009). It is reasonable to suggest that the stress experienced by these students was caused in part by the uncertainty that the loss of their secondary school "dwellings and possessions" and the transition to an unknown future engendered.

\section{Schlossberg's Transition Theory.}

Schlossberg's Transition Theory is a widely recognized model that seeks to provide a systematic framework for helping understand how individuals experience transition and how they can cope with such transitions (Evans, Forney, Guido, Patton \& Renn, 2010). Though often considered an adult development theory, it is equally relevant to students of college age (Evans et al., 2010) and has been suggested as a valuable model for defining the transition experience for many students identified with autism (Lee, Mathur, McCoy and Zucker, 2014).

Schlossberg (1981) posited that a transition has occurred when "an event or nonevent results in a change in assumptions about oneself and the world and thus requires a corresponding change in one's behavior and relationships" (Schlossberg, 1981, p. 5). Developed over time as the result of additional research by herself and others as well as 
critiques of earlier versions, Schlossberg (2011) posited that transitions are either anticipated (e. g., the movement from secondary school to a postsecondary environment), unanticipated (e. g., the sudden loss of a family member), or nonevents (e.g., not being admitted to your preferred postsecondary institution) (Schlossberg, 2011). In addressing these transitions, the individual would adapt based on the interaction of their perception of the event, the characteristics of the pre-transition and post-transition environments in play, and their own characteristics.

Drawing on the work of others (Bridges, 2004; Bloch and Richmond,1998; Bright \& Pryor, 2008; Bussolari \& Goodell, 2009; Hudson, 1991, 1999), Anderson, Goodman and Schlossberg (2012) posited an integrative model of the process of transition involving three stages during which one moves into, through and out of a transitional event (Figure 1).

Figure 1. Graphic representation of Schlossberg's (2012) Transition Theory (Anderson, Goodman and Schlossberg, 2012)

Integrative Model of the Transition Process

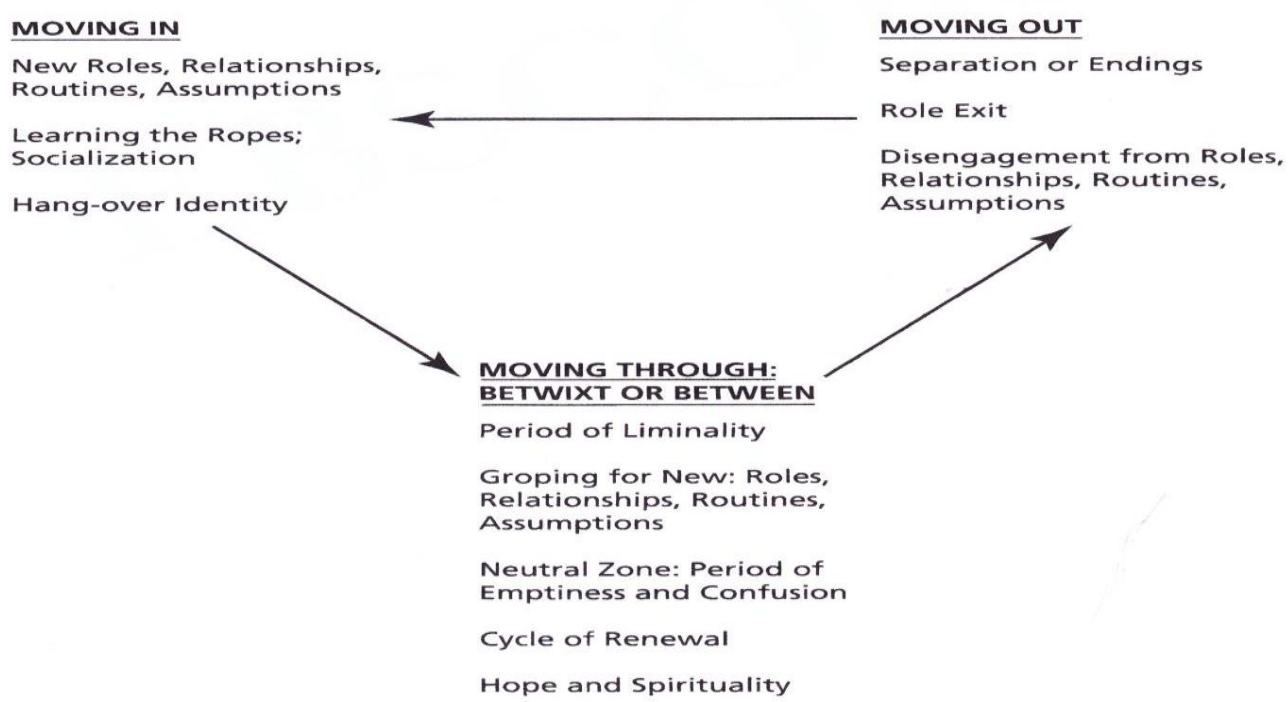


Anderson et al., (2012) further offered that four factors influence how one managed transition. These factors are situation (the characteristics of the transitional event or non-event, such as timing, duration and control), self (one's personal characteristics and resources for dealing with change), support (one's social network, such as parents, peers and institutional support) and strategies (one's available responses for coping). Known as the "4S Model", these variables can be viewed as potential assets or liabilities, taking on greater or lesser significance depending on the nature of the transition faced and which factors one leverages in addressing the transition. (Anderson et al., 2012).

Lee, Mathur, McCoy, and Zucker (2014) stated that although Schlossberg's Transition Theory had heretofore been utilized primarily for either young children or adults, it was "very relevant to the transition of ASD students of all ages" (Lee et al., 2014). The authors further suggested that Schlossberg's theory was valuable in understanding the transition to postsecondary institutions by stating that the theory was especially relevant in instances where a collegiate circumstance (such as open seating) might constitute a significant disruption in routine for a student with ASD, but would barely register for a "neurotypical” (NT) student (Lee et al., 2014). Schlossberg's theory has the potential to further benefit students with ASD as they experience transition, in that it specifically identifies the key components necessary for success, identifying the self as a primary source of liability for students with ASD due to their general preference for routine (Lee et al., 2014). Recognizing this liability, Schlossberg's model called for the reinforcement of the factors of situation, support and strategies as compensation. The willingness of students identified with autism to accept parental or peer 
mentoring/support (Ebel, 2015; Giarelli, Ruttenberg, \& Segal, 2013; Mitchell and Beresford, 2014; Peña and Kocur, 2013), the willingness of peers to provide such support (Neville and White; 2011), and the recognition that such support results in more successful transitions (McCoy, 2014), offered hope that such strategies, as well as other inclusive transitional programs at the institutional level would be successfully implemented.

\section{Rendón's Validation Theory.}

Rendón's Validation Theory (1994) was originally developed in response to the experiences of culturally diverse, low income, or first-generation students enrolled in postsecondary education. Rendón built on the work of Astin (1985), who posited that students learn more as they become involved through investing physical and psychological energy in activities, people, and tasks, and Pascarella and Terenzini (1991), whose review of research found that casual interactions with peers and faculty members, and participation in extra-curricular activities, were important in shaping positive attitudes and values towards learning. Rendón (1994) presented her theory as a mechanism for assisting minority and other nontraditional students in achieving college persistence through validation. Through intentional, proactive affirmation, family members, peers, faculty and staff academically and interpersonally validate the students as valuable members of the academic learning community, while fostering individual growth and social acclimation. The author considered this theory to be applicable and of significant utility to students identified with autism as they navigate the transition from secondary to postsecondary school. 
Validation Theory as presented by Rendón (1994, 2002, Rendón Linares and Muñoz, 2011) consists of six elements. The first element places responsibility on faculty and staff to actively initiate contact with students in "authentic, caring and nonpatronizing ways" (Rendón Linares \& Muñoz, 2011, p. 18). The second element declared that the result of a validating interaction should be to confirm to the student that they bring valuable knowledge to the academic setting and can succeed. The third element posits that when students are regularly validated, they are more likely to feel confident in the academic setting. Fourth is that the process of validation is not limited to the classroom but can and should occur on a consistent basis campus wide. Fifth is the idea that validation is not a single event, but rather a process that begins early and continues throughout the collegiate experience. Finally, validation is most critical "when administered early in the college experience, especially during the first few weeks of class and the first year of college" (Rendón Linares and Muñoz, 2011, p. 18).

Though Rendón did not specifically address students with autism in her research, it seemed that her approach as delineated would substantially assist a number of these students in their successful transition to a postsecondary educational institution. Her sixth element, advising early administration of validation techniques would especially align with transitional practices. Furthermore, research has shown that validating strategies are effective with students identified with autism (Brown \& Coomes, 2016; Ebel, 2015; Gobbo \& Shmulsky, 2012; Kelley and Joseph, n.d.; Koegel, Ashbaugh, Koegel, Detar \& Regester, 2013; Peña and Kocur, 2013; Shmulsky, Gobbo and Donahue, 2015). Providing the mechanisms and support necessary to foster validation for all students, including those identified with autism, cannot help but promote integration into 
the collegiate social system, and thus lead to increased persistence, academic success, and individual development for the entire student population.

\section{Bean and Metzner's Attrition Model.}

Bean and Metzner (1985), recognized that the early work on persistence/retention conducted by Spady (1970), Tinto (1975), and Pascarella (1985), was heavily dependent on social integration within the collegiate system, yet for the rising number of nontraditional college students (older, part-time and commuter students), such social integration was less likely to occur; a theoretical model to explain attrition for these students was needed. Bean and Metzner (1985) devised the Attrition Model, which indicated that decisions relative to attrition would be primarily based on four sets of variables: academic performance, intent to leave (a function of psychological and academic variables), background variables (e. g., high school performance, educational goals), and environmental variables (e.g., finances, family responsibilities) (Figure 2). The four variables were viewed as compensatory; a shortcoming in one variable could be overcome by a strength in another variable. They also contended that an understanding of the forces in play could allow one to calculate the likelihood of attrition by calculating the direct and indirect effects of the individual factors.

While the author might question the degree to which Bean and Metzner could accurately calculate attrition likelihoods, their model was included in this discussion because it attempted to address the experiences of non-traditional students. Previous research (Adreon and Durocher, 2007; Perner, 2002) recommended that students identified with autism consider living at home during the first year of college to minimize the social challenges of the transition from high school while they addressed academic 
and collegiate challenges. Living at home would provide the social supports to which they are accustomed and identify these students as non-traditional as defined by Bean and Metzner. As proposed by the Attrition Model, this would offer the compensatory support needed to overcome any shortcomings present elsewhere as illustrated in the model.

While not a general approach to addressing the needs of students identified with autism, the Attrition Model does provide guidance within the context of living-at-home, first-year college students.

Figure 2. Graphic representation of Bean and Metzner's (1985) Attrition Model (Aljohani, 2016)

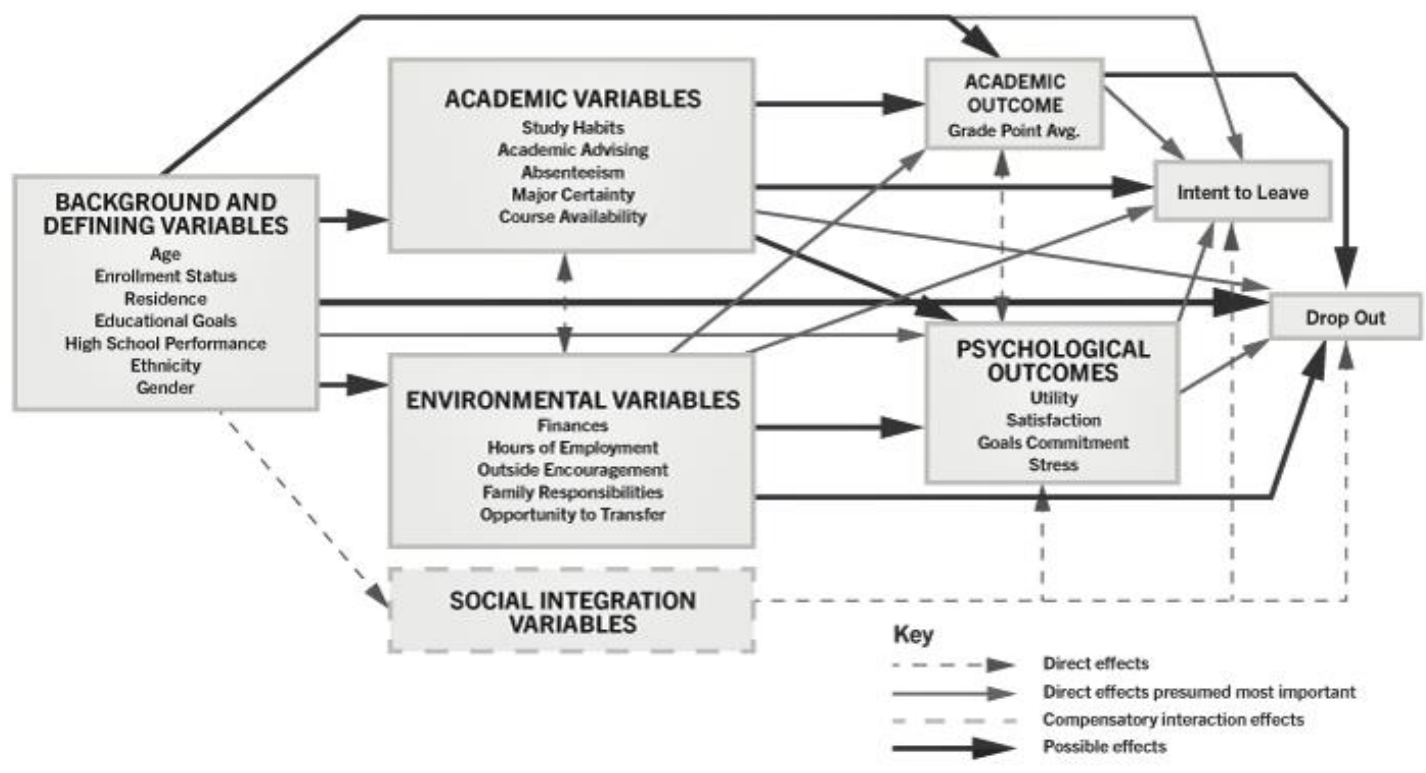

\section{Models of transition and the student identified with autism}

The purpose of this consideration of theoretical models relative to transition for students identified with autism was to clarify the current terrain of transition in a way that guided the research towards an authentic and useful understanding of the experience for 
these students. Through consideration of these theories and models, the author was able to construct the study in a fashion that "got the most of out the process".

The models of transition provided a wealth of understanding for the researcher. Morrison and Clark's theory (2016) provided what the researcher views as a key understanding: the first step in the transition process is a disengagement from secondary school. This process can be stress inducing and should be addressed as a distinct transition as well as a component of the overall transition. Schlossberg's $(1981,2011)$ theory of transition provided a structured approach to understanding both the stages of transition and the factors that affect one's success in the transition. Rendón's theory (1994), originally developed for a culturally diverse, low income population, was included because all students, including those identified with autism, have a need for authentic validation and affirmation. Providing a universal program of validation through proactive affirmation benefits all students, including those identified with autism. Finally, Bean and Metzner's theory (1985) was included because it focused on nontraditional students, including commuter students. As researchers have recommended that students identified with autism may benefit from living at home during the first year of transition (Adreon and Durocher, 2007; Perner, 2002), their theory seemed appropriate for inclusion. Taken together, the work of Morrison and Clark 2016), Schlossberg (1981, 2011), Rendón (1994) and Bean and Metzner (1985) formed a bricolage of established research that informed the current study.

\section{Background: Autism by any other name...}

Ever since Wing (1981) familiarized academia with the foundational work of Hans Asperger, medical model researchers have regularly revisited the devising of a 
proper term to be used to describe individuals with average to above average cognitive functioning but deficits in socio-communicative skillsets. Initially, "Asperger Disorder" (AD) or "Asperger Syndrome" (AS) were utilized, with AS officially acknowledged within the American Psychiatric Association's (APA) Diagnostic and Statistical Manual IV (DSM-IV) in 1994 (Klin \&Volkmar, n.d.). AS was incorporated into the more global term, Autism Spectrum Disorder (ASD) in May of 2013 (APA, 2015).

Concurrent with these changes in terminology was a profound increase in reported cases of autism, from early prevalence rates of less than 1 per 1,000 (Wing \& Potter, 2002; Matson \& Kozlowski, 2011) to the current rate of 1 in 59 (CDC, 2018). Whether these numbers represented an actual change in the incidence of autism or whether they are the result of what researchers have termed diagnostic migration (Cardinal \& Fraumeni-McBride, 2017), the reported increase in incidence has likely resulted in an increased interest surrounding the field, and a resultant call for scholarly research.

Between 1994 and 2013 such research occasioned findings being reported that encompassed individuals who would today be medically classified as having ASD, but at the time were specifically classified as having AS or AD. As a result, the current body of research utilized multiple terms including "Asperger Disorder” (AD), "Asperger Syndrome" (AS), "High-Functioning Autism" (HFA) and "Autism Spectrum Disorder" (ASD). These terms have a medical origin and as such negatively affect identified individuals by subjecting them to varying degrees of stigmatization and othering. The researcher eschewed these terms as hegemonic and disabling, yet the research conducted 
during periods when these terms were in vogue provided necessary perspective and insight.

In contrast to the medical model origin of identifiers, it was reported that within the autism community many self-advocates prefer the use of the term "autistic" or a variation thereof. This was because they perceive autism as an inherent part of the identity of the individual, in the same way one might describe an individual as tall, athletic, or blonde (Brown, 2011).

Given the range of terminology utilized, a cohesive approach was a necessity: For this study, the intent was threefold; to be as inclusive of "the current body of knowledge" as was useful, to communicate succinctly while respecting the individual preferences of the participants, and to address the central question of transition for "students identified with autism". Scholarly rigor demanded that I considered research couched in these terms, yet succinct communication necessitated the use of terminology which is universally understood and accepted.

For these reasons, when referencing research, terminology originally used in the research to identify the specific population considered was employed. When discussing individual students, each student was identified utilizing terminology that they themselves selected. When discussing the student participants in general, "students identified with autism”, a widely accepted, balanced, social model appropriate and person-first moniker was utilized.

\section{Basic Assumptions}

It was necessary that the investigator adopt three assumptions to conduct research into the perceptions of students identified with autism and other key participants 
regarding transition from high school to community college. Each participant selfidentified as a member of a key participant group; students self-identified as having autism, parents self-identified as parents of students with autism, faculty, staff and other key participants self-identified as such. The most basic assumption pertinent to this study was that the study participants accurately reported their perceived experiences, responding to the interview questions fully and truthfully. For this study, truthful answers were not merely a matter of the absence of obfuscation; to answer truthfully, participants needed to possess the capacity for truthful responses. The investigator must assume that the participants fully understood the questions, processed the questions within their own cognitive framework, and articulated responses that properly reflected their intent. Finally, it was assumed that various methods used in conducting the study accurately gauged the factors intended to be evaluated.

\section{Basic Limitations}

While the researcher conducted interviews with students, parents, faculty and staff, no one group of participants predominated; neither was any group represented to the extent that findings regarding those groups could be transferable. Furthermore, the transition experience studied took place within the context of an affluent suburban community college and its feeder secondary school. Findings should be considered relevant only within that delineated setting. Within that defined and stratified setting, the researcher intended that the findings represent an introductory, unified understanding of transition for students identified with autism; the development of a comprehensive unified understanding must be the life's work of future generations. As such, the findings 
uncovered from this study were directly applicable only to this study and should not be applied to a wider audience.

The participants for this study were predominantly convenience obtained; personal contacts at the secondary and postsecondary schools were utilized to provide access and introduction to the individuals who ultimately participated in the study. As such, the study population is not to be perceived as being representative of the larger population.

\section{Summary}

This chapter introduced an exploration of the experiences and perspectives faced by students identified with autism, their parents/caregivers, high school and community college faculty and other stakeholders relative to these students and their transition from high school to community college. A consideration of the central questions and the significance of the problem, as well as overarching theories and models influencing the course of the study were addressed. In addition, a brief review of the changing verbiage relative to autism, as well as basic assumptions and limitations were discussed. 


\section{Chapter 2: Literature Review}

This review delineated current findings from the literature that addresses students identified with autism and their transition from high school to college. In doing so it provided an appropriate academic backdrop for the consideration of students with autism and their challenges in transitioning from secondary school to a community college.

In the 1990s, changes in guidelines for diagnosis of ASD and increased public awareness led to a significant increase in numbers of children identified as having autism (Vanbergeijk, Klin and Volkmar, 2008). National statistics reflected these increases, as

evidenced by a change in identified prevalence of ASD from 1 in 150 for children born in 1992 to an identified prevalence of ASD of 1 in 59 for children born in 2006 (CDC, 2018). Although individuals identified with autism may have a coexistent intellectual impairment as well, it was reported that $44 \%$ of the population of individuals identified with ASD have average to above average cognitive capabilities (CDC, 2018). These statistics suggested that there were likely many secondary school students identified with autism who possessed the cognitive capabilities necessary for a successful transition to post-secondary education. Indeed, as a likely result of improved educational programs at the K-12 level and greater access to accommodations, these students were enrolling in greater numbers at the post-secondary level (Adreon \& Durocher, 2007).

Given this increasing enrollment, it is incumbent upon researchers to identify the factors that promote a successful transition as well as those that facilitate not only learning, but true integration into an inclusive, collegiate society. To this end, this literature review addressed two questions: 
1. What are the individual, family and secondary school factors that promote a successful transition from secondary school?

2. What are the individual, family and postsecondary school factors that promote a successful assimilation to an inclusive, postsecondary educational environment?

Though the researcher explored transition to a California community college, little research addressed that domain specifically. Consequently, this literature review addressed postsecondary settings generally, with the intent of culling from the findings those factors that may especially influence those transitioning to California community colleges specifically. Additionally, though the dissertation was approached from a unified perspective, the preponderance of literature addressed transition either from the secondary or postsecondary perspective. Yielding to the predominant paradigm, the researcher thus presented the literature review from that perspective.

Utilizing the words, "autism," "college" and "transition" as terms to be found in a title, an advanced digital search of the EBSCO system was conducted. This netted 90 results. Whereas a number of these sources failed to address the two key questions or provide appropriate enhancement, mining their references led to additional sources which were of use. Ultimately, 44 sources were utilized in the development of this review of literature.

Literature included in this review covered over two decades of research both in the United States and abroad. As a result, several terms were used to identify students who are following a collegiate trajectory and are identified with autism. These terms included Asperger's Syndrome (AS), Asperger's Syndrome Disorder (ASD) and High- 
Functioning Autism (HFA). Given the range of utilized terminology, a cohesive approach was required.

Utilizing the threefold intent of inclusiveness, clarity and focus as specified in Chapter 1, the researcher utilized original terminology when referencing specific literature. When discussing the global population under consideration, "students identified with autism", a widely accepted, balanced, social model appropriate and person-first moniker was utilized.

\section{Why Transition to College?}

Tangential to the understanding of factors impacting students identified with autism and their transition to postsecondary educational settings was understanding the impetus towards postsecondary enrollment. According to Humes (2006), prior to World War II, attending a four-year institution was generally limited to the elite in society. With the signing of the Servicemen's Readjustment Act of 1944, President Franklin Roosevelt made a four-year, postsecondary education within reach of the middle class. The response was staggering; literally hundreds of thousands of World War II veterans utilized the program to become teachers, doctors, engineers and scientists who were unleashed within every sector of the economy, resulting in dramatic upward shifts in living standards for all. As a result, society realized that a highly educated population was beneficial to America, and college became an ingrained aspect of the American Dream (Humes, 2006).

This preference for college has continued unabated since the postwar era. As recently as 2014, the Pew Research Center reported that young college graduates outperformed their less educated peers on virtually every standard of "well-being and 
career attainment-from personal earnings to job satisfaction to the share employed full time" (Pew Research Center, 2014). Given that more and more students identified with autism are graduating from secondary school with the capability of enrolling and succeeding in a postsecondary institution, it is reasonable that they would do so to obtain the same benefits that recent research identifies.

\section{Factors that Promote Transition from Secondary School for Students Identified With Autism}

\section{Individual Factors}

In terms of individual factors that promote a successful transition for students identified with autism from secondary school to a postsecondary setting, scant research has been conducted and that research generally provides findings that are unremarkable in that they are consistent with those expected of the general population. For example, data analysis of the NLTS2 by Chiang, Cheung, Hickson, Xiang, \& Tsai (2012) identified the student's academic performance as a key indicator of participation in postsecondary education for students with ASD (Chiang, Cheung, Hickson, Xiang, \& Tsai, 2012). Giarelli, Ruttenberg, \& Segal (2013) studied adolescents with AD aged 18-23 and reported that students who could identify special intellectual abilities (e.g., technical skills, memory for details, numbers) and possessed strong verbal skills were more likely to experience success in transition to the adult community. Giarelli et al., (2013) also reported that adolescents were more likely to transition successfully if they possessed specific personal qualities, including a caring, empathetic manner, a tolerant and cooperative outlook, and the capability to advocate for oneself (Giarelli et al., 2013). While these results could well be anticipated, they are useful in that they suggest that 
secondary programs that foster the academic and emotional strength of all would benefit all, including those identified with autism.

Considering self-concept and its influence on transition, research by Browning, Osborne and Reed (2009) indicated that as students with ASD approach the conclusion of their secondary school experience, they perceive themselves to be less able to deal with stress and are unable to identify the reasons why. Further, students identified with autism often responded to stress with internal discomfort rather than displaying outward signs of stress. For this reason, it can be especially difficult to recognize (Browning et al., 2009). Given the "hidden" nature of this stress, secondary institutions would do well to incorporate strategies for coping with stress into a general program of emotional wellbeing or provide such training as a stand-alone component of a secondary school's mission. This would address such hidden needs and benefit all students.

The two studies and one data analysis conducted to determine individual factors promoting transition for students identified with autism represents an extreme paucity of research. The two studies utilized a total of 30 participants, primarily white and male. The data analysis report studied a large population, with no mechanism for determining the number of students identified with autism who were following a college trajectory. That the results from the three studies were consistent with those one might obtain from a generalized population supports the recommendation that addressing these factors should be conducted through a universal program at the secondary level.

\section{Family Factors}

The importance of support by family and others is highlighted by the research conducted into factors that promote successful transition. For example, Mitchell and 
Beresford (2014) conducted research that found that parents can serve as important support mechanisms for educational transitioning for their adult children. Conducting interviews with students identified with HFA in England, the research revealed that the students acknowledged their need for an individual to assume a coordinating or administrative role as well as the need for emotional support through the transition process. The report stated that many of the students interviewed identified parents as the individual(s) providing the administrative and emotional support (Mitchell \& Beresford, 2014). The need for an assisting individual is echoed by the research of Giarelli et al., (2013), who identified the role of a "mentor" as perceived by adolescents identified with autism to be a desirable component of their transition. Individuals reported that a mentor would provide valuable guidance through modeling appropriate behaviors, aiding in times of difficulty and offering tolerance and understanding (Giarelli et al., 2013). Some research also indicated that the use of a peer-mentor could have the benefit of reducing stress as related to the process of transition (Cochran, 2009).

These three studies were comprised of predominantly white males, with no data provided as to socioeconomic level. With a combined population of 37 , the findings should be considered tentative. Still, the support of family and others, whether as administrators, comforters, or mentors is an approach which would seem beneficial to all students. Such services, provided through a school's counseling center, peer guidance programs, or as part of a school-wide initiative, are minimally invasive, community driven and not deficit-based. 


\section{School Factors}

Literature offering specific recommendations for implementation by secondary school institutions tends to be generalized in nature and more the result of professional opinion than scholarly research. While professional opinion certainly has merit, a more pronounced path from research to recommendation is warranted.

The central thrust of the literature into school factors that promote a successful transition focuses on the benefits of a formalized transition program for students identified with autism. Based on analysis of NLTS2 data, students identified with autism who participated in transition programs and developed postsecondary goals were more likely to enroll in a postsecondary educational institution (Wei, Wagner, Hudson, Yu, \& Javitz, 2016). Roberts (2010) offered the opinion that a transition program should incorporate a full range of features including specific postsecondary coping skills (e.g. time management, self-advocacy), exploration of possible careers and academic goals and an assessment of the student's learning style (Roberts, 2010). Despite this research, survey responses of postsecondary New York state college disability coordinators led Janiga and Costenbader (2002), to report that some students, including those identified with autism, may not have received adequate attention to their transition planning due to a focus on students with more severe physical or emotional disabilities (Janiga \& Costenbader, 2002). Based on the research of others, Levinson and Ohler (1998) opined that students identified with autism who were following a postsecondary academic trajectory may not receive adequate guidance and planning because of a staff misconception that their "condition" is so mild that they can succeed in college without support (Levinson \& Ohler, 1998). 
These investigators highlighted a central failing of most secondary school programs as relates to transition: coursework is provided to prepare students to meet the academic requirement of admission to a postsecondary institution, but actual guidance and transition skill-building is parsed out based on categorizing students into priorities of perceived need. By rethinking secondary school programs to provide guidance for all, we facilitate transition not just for those who have been identified as needing such guidance, but all students who might benefit from some level of transition support. Some literature proffers specific recommendations for transition guidance. Utilizing the NLTS2, Chiang et al., (2012) developed four recommendations to assist secondary school teachers in facilitating postsecondary education for students with ASD: (1) Ensure that students with autism are educated within the general student population utilizing the standard curriculum to the greatest extent possible; (2) Ensure efficacious instructional practices to maximize academic performance; (3) Develop and maintain ongoing partnerships with parents to promote postsecondary education; (4) Include the goal of attending postsecondary school in a student's Individualized Education Program (IEP) as early as is appropriate (Chiang et al., 2012). Szidon, Ruppar, and Smith (2015) identified the importance of the IEP as well, recommending that it include five specific steps that address transition to a postsecondary education. These steps included: (1) Identifying specific transition goals through assessment, (2) Linking these postsecondary goals with the IEP goals in an authentic fashion, (3) Ongoing monitoring and adjustment of these goals, (4) Providing the classroom teacher with the time and training to teach these goals, and (5) Periodic evaluation of the efficacy of these steps (Szidon, Ruppar, \& Smith, 2015). While the recommendations of Chiang et al, (2012) and Szidon et al, 
(2015) were reasonable professional guidance to be applied within the confines of the IEP, they function within the medical model perspective in that they focus on that population that has been observed, tested and identified as being within the special education arena. This approach has the potential to miss a significant component of the secondary school population; students identified with autism who are pursuing a collegiate trajectory but do not fall under the IEP mechanism. By establishing schoolwide programs of goal establishment, instructional rigor, parental partnerships, and ongoing review, all students can benefit from a robust transition program.

It is sometimes the case that the counseling department is given responsibility for ensuring that the transition practices of a secondary school are rigorous. Recognizing that "There are limited school counseling resources that address the unique post high school transition issues faced by students identified with High-functioning Autism Spectrum Disorder" (p. 2), Dipeolu, Storlie and Johnson (2014) authored a document designed to provide focused strategies for counselors in assisting students with ASD in transitioning to college. Reviewing the work of other researchers, they determined that strategies that improve social skills, reduce anxiety, improve test taking and facilitate planning for postsecondary transition success were both effective and within the capacity for counselors to implement. They further advised counselors to take specific actions, including getting to know the student, working with the family and other school professionals as a team, building on the personal strengths of the student, and reaching out to the community for assistance (Dipeolu, Storlie \& Johnson, 2014).

While the findings of Dipeolu et al. (2014) are of note, this researcher contends that counselors generally function under caseload and time constraints that would make 
personally conducting such a program untenable. It could also have the effect of identifying and thereby "othering" a segment of the school population. A more practical approach would be to train the faculty and staff to incorporate such strategies into their daily programs for all and provide buttressing for those students desiring additional support.

Zager and Alpern, (2010) recommended that secondary schools develop partnerships with local colleges to allow students with ASD to attend secondary classes on postsecondary campuses. This would serve as an effective means of decreasing stress regarding transition to college among students on the autism spectrum. This approach has merit, though it would be limited to those schools in reasonable proximity to postsecondary campuses and would require an accommodation for transportation. Another approach might be to offer introductory, required postsecondary courses on campus, taught by a college instructor, and make them available to any student who wished to participate. By doing so, all students have equal access and "othering" is minimized.

Zager and Alpern (2010) inadvertently alluded to one of the central challenges of transition from secondary school to a postsecondary educational institution for all students when they noted that secondary school is a completely discrete and independent institution from postsecondary school. While these two institutions communicate from time to time, requesting transcripts or offering recruitment presentations, insufficient formal coordination occurs. As a result, what transpires is less a transition and more a disengagement with a subsequent engagement in a different venue, a more challenging process requiring more intense support, support that is not currently provided. 
The literature offered for secondary institutions is highly derivative; most of the material consisted of professional recommendations based on compilations of previously published work. What little research was conducted utilized the NLTS2, resulting in challenges identifying which students were pursuing a collegiate trajectory, as well as an inability to identify age, gender, ethnicity or socioeconomic level. This is an area where scholarly research is especially needed.

Factors that Promote Success for Students Identified with Autism as they Transition to a Postsecondary Educational Environment

\section{Individual Factors}

In transitioning to a postsecondary educational setting, the literature suggested that several factors related to the individual come into play. These include the choice of educational setting, degree of independent living skills, acclimation to changes in sensory input, self-advocacy skills, and socialization skills leading to peer acceptance. These factors are further examined in the following.

Choice of educational setting. Adreon and Durocher (2007) addressed the matter of choosing a postsecondary educational setting. They recommended that the student identified with autism should consider the type of college (e.g., technical school, community college, four-year college), school enrollment, class sizes, configuration of the campus and proximity to home (Adreon \& Durocher, 2007). Regardless of the type of school, they advised that choosing an institution of the appropriate size can especially influence success for those with ASD (Adreon and Durocher, 2007). A smaller school can provide the student with ASD a more navigable, less engulfing setting but can 
conversely result in the student being more visible within the school community and less likely to find others with similar interests (Adreon and Durocher, 2007).

Adreon and Durocher (2007) intended that the postsecondary institution chosen be large enough for ample social interaction while small enough for the institutional community to provide comfort and security. Community colleges are ubiquitous and often smaller than state colleges and universities, and thereby provide institutional comfort and security while offering a better opportunity to attend college while still living at home. Deciding whether to attend a local institution or enroll far away are decisions faced by many transitioning students; secondary schools should provide guidance for all their students that addresses these concerns.

Degree of independent living skills. Integral to the school selection process is the extent of independent living skills that the individual possesses. It is self-evident that the further from one's family a student resides, the more likely they are to need to address daily living issues without parental support and guidance. Researchers have noted that individuals identified with autism may experience difficulty with necessary daily living skills, such as waking up to an alarm clock or addressing personal hygiene (Adreon, 2016), getting to class on time (Adreon, 2004), shopping (Prince-Hughes, 2004), navigating public transportation, or ensuring personal safety (Adreon, 2016). If a student identified with autism chooses to attend a postsecondary educational setting some distance from home, their independent living skills must be equal to the challenge. Perner (2002) advised attending school near home, at least for the first year, to allow the student identified with autism to adjust to the changes in educational setting without the added complication of adjusting to living changes as well (Perner, 2002). Mentoring by 
parents (Mitchell \& Beresford, 2014; Giarelli et al., 2013) or peers (Cochran, 2009) can provide valuable assistance in adjusting to this transition.

Prince-Hughes (2004) and Adreon \& Durocher (2007) have noted that an individual's organizing, problem-solving, and decision-making skills play a role in successful independent living. Executive function, defined as the cognitive mechanisms needed to successfully perform goal-directed behaviors, often varies considerably within the population of individuals identified with autism (Prince-Hughes, 2004; Adreon \& Durocher, 2007). Researchers have identified that tools designed to specifically assist in organization and planning (e.g. calendars, checklists, day-planners) may provide valuable assistance in enhancing a student's executive functioning (Robertson \& Ne'eman, 2008). In addition, literature has proffered that technology (e.g. computers, smartphones, calculators) may especially appeal to the cognitive strengths of students identified with autism (Moore, McGrath \& Thorpe, 2000; Ferguson, Myles, \& Hagiwara, 2005) or aid in the development of social skills (Mitchell, Parsons, \& Leonard, 2007). Devices that aid in organization and planning, especially those of a personal, technological nature, can offer non-invasive support on an as-needed basis.

Acclimation to changes in sensory input. In considering the readiness of an individual with autism for living away from home, it is important to consider the individual's ability to acclimate to changes in sensory input, as this can affect adjustment to the college environment. Students with autism may process sensory input in their own fashion. They may experience a heightened or lessened sensory experience that can vary greatly depending on an individual's stress level, emotional condition or environment (Gillot \& Standen, 2007). Sensory issues may present themselves as sensitivity to sounds 
and smells (Adreon \& Durocher, 2007), or a heightened sensitivity to the feeling of furniture, lighting, or tastes (Prince-Hughes, 2002). According to Perner (2002), living away from home can be difficult if the individual with ASD is especially reactive to sensory input. The student's reactions to a noise in the dorm, the feel of a different bed, or the taste of food in the cafeteria can result in significant interference with their adjustment to the postsecondary educational setting (Perner, 2002). To address this, the postsecondary institution can take measures to ensure that the educational setting, including the housing and dining accommodations, are designed in a manner that is amenable to all.

Self-advocacy skills. Self-advocacy is the ability to understand one's own needs and communicate those needs effectively to others (Shore, 2003). Postsecondary educational success can be especially influenced by the facility of a student to advocate for oneself (Adreon \& Durocher, 2007). Whereas secondary school is designed with the understanding that students are minors and lack the legal standing to self-advocate, postsecondary educational settings presume adulthood on the part of the students and expect them to be able to advocate for themselves (Highlen, 2017). Students identified with autism who are attending a postsecondary institution typically must be able to disclose their condition to teachers and others as a prerequisite to requesting specific supports and accommodations (Adreon and Durocher, 2007). An inability to adequately advocate for supports and accommodations makes obtaining said supports and accommodations less certain, thereby affecting postsecondary educational outcomes (Roberts, 2010). 
This need to self-advocate can be addressed in several ways. Programs that teach self-advocacy can be incorporated throughout the curriculum at the secondary level, thereby ensuring that all students have the skillset necessary to advocate on their own behalf. Postsecondary institutions can ensure that faculty and staff are educated to this need and take actions that foster an environment where self-advocacy is encouraged. In addition, these institutions can restructure classroom instructional practices such that inclusive practices are utilized for all, thereby minimizing the need for stigmatizing specialized accommodations for some.

Peer interactions/acceptance. Students identified with autism may display a range of social impairments and idiosyncratic behaviors that can affect peer interactions and acceptance (White, Ollendick, \& Bray, 2011). According to Sayman (2015), these would include "poor eye contact, diminished facial recognition, awkward body movements, challenges interpreting body language, impaired social interactions, and difficulty with organization" (Sayman, 2015). A systematic review of the literature by Gelbar, Smith and Reichow (2014) unearthed 20 studies involving 69 people and reported that the inability to integrate socially within the collegiate environment can lead anxiety, loneliness, depression, isolation, and marginalization (Gelbar, Smith, \& Reichow, 2014).

Researchers have identified strategies that improve peer interactions for students identified with autism. Müller, Schuler and Yates (2008) reported that structured social activities, social facilitations, and modeling of socially appropriate behaviors are methods that have been effective with adults and could be modified as appropriate for college-age adults. Other strategies, such as providing a list of interest-specific social events to 
attend, developing time-management strategies to encourage attendance at these events, socialization mentors, and weekly meetings to discuss social events may also benefit these students (Koegel, Ashbaugh, Koegel, Detar \& Regester, 2013). Wallace (2016) reported that students with ASD identified role-playing to enhance socialization skills, curricular assignments requiring volunteer work, and organized group socialization opportunities as especially useful strategies.

The extant literature addressing factors affecting individuals in their transition demonstrates the dire need for further research. Current literature is derived from a range of venues utilizing a spectrum of investigational strategies. The professional guidance offered by Prince-Hughes $(2002,2004)$ and Robertson \& Ne'eman (2008), is enhanced and made authentic by their self-identification as individuals with AS or autism respectively. Others proffered recommendations based on the study of single individuals (Ferguson, Myles, \& Hagiwara, 2005; Sayman, 2015). The work of Gillot and Standen (2007) was of note in that they utilized a relatively large population, matched by age, gender and intellectual ability, in comparing 34 adults with autism to 20 adults with intellectual disabilities to determine that those identified with autism are nearly three times more likely to experience anxiety.

Beyond the numbers, few studies offered much in the way of demographic data. Ferguson, Myles, \& Hagiwara (2005) offered that their study was based on one midwestern white male middle schooler with "above average" (p. 61) cognitive skills. The findings of Koegel, Ashbaugh, Koegel, Detar \& Regester, (2013) were based on the study of three college-age males, two "Euro-American" (page 901) and one Latino. Mitchell, Parsons, \& Leonard (2007) noted that they studied four male and three female 
middle schoolers with diagnoses of ASD. The review of previous studies by Gelbar, Smith and Reichow is especially illustrative; of the 69 participants studied, 41 were identified as male, nineteen were female, and nine were unidentified. Only 25 of the 69 participants were identified by age. No data was provided as to ethnicity or socioeconomic status.

These challenges within the literature can have the effect of throwing doubt on the veracity of the recommendations. Beyond the doubt, many of these strategies have the effect of separating and grouping students identified with autism to obtain specialized assistance. Even the use of socialization mentors establishes the mentor in a privileged status. To address this, programs need to be organic to the collegiate experience, providing guidance in the most unobtrusive manner practicable.

\section{Family Factors}

Though adulthood severs the legal rights and responsibilities previously held by a parent, individuals identified with autism who are attending a postsecondary educational setting can still benefit from parental involvement. This was supported by Peña and Kocur (2013), who interviewed eighteen parents of students with ASD who attended community college. Parents reported that their two primary functions were supporting students in navigating campus services and encouraging independence in the college setting (Peña \& Kocur, 2013). Ten parent interviews conducted by Ebel (2015), identified the importance of their ongoing advocacy for their children throughout the postsecondary school experience, as well as the need to develop and maintain lines of communication and support between the schools, their children and themselves (Ebel, 2015). 
In addition to organizational challenges, adjusting to the social demands of the college setting can be taxing for students identified with autism (Welkowitz \& Baker, 2005). Given these challenges, researchers have suggested that it is helpful for many students identified with autism to live at home the first year, so that they can acclimate to the academic and organizational demands of college, before adding social and living challenges (Adreon and Durocher, 2007; Perner, 2002).

Of the eighteen parents interviewed during the Peña and Kocur (2013) and Ebel (2015) studies, only the ten studied by Ebel (2015) were identified by gender (nine females and one male) and ethnicity (nine Caucasian and one African American) (p. 80). None of the parents were identified by age or socioeconomic status, though Ebel (2015) did identify the occupation of the parents. Additional information on the demographics of the parents would have been desirable.

American society has established through legislation that an individual becomes a full-fledged adult on their eighteenth birthday. Yet a practical understanding of youth and maturation argues against such an instantaneous metamorphosis. Students at postsecondary institutions can generally benefit from the support and guidance of parents and others, and institutions can foster such involvement through the development and maintenance of a welcoming, inclusive environment.

\section{School Factors}

Institutional approaches. The recommendations of Kelley and Joseph (n.d.) provided a practical approach for community colleges to aid in the transition of students identified with autism. Leveraging their capital as practicing community college faculty, they suggested that the school's Disability Services office develop, in coordination with 
the student affairs department, a summer program to facilitate transition for these students into the collegiate setting. They further recommend that this summer program transition to a structured, first-year program that maximizes success by providing academic and social supports and peer assistance (Kelly \& Joseph, n.d.). This was echoed by Shmulsky, Gobbo and Donahue (2015), who advised that successful transition relies on early contact with parents and students, small-group orientations to facilitate student adjustment, and ongoing first-year support (Shmulsky, Gobbo \& Donahue, 2015). Others advised that campus support offices collaborate with community resources to tailor mechanisms which best support the individual needs of students identified with autism (MacLeod \& Green, 2009: Roberts, 2010), or recommend programs intended to bridge secondary school and the postsecondary educational setting through a two-step approach that emphasizes self-determination and self-regulation (White et al., 2017).

Secondary and postsecondary institutions have traditionally functioned as discrete entities, nominally reliant on one another but with the expectation that students will "make the jump" from one to the next essentially by themselves. These researchers recognized that such an approach impedes many students; by developing mechanisms that promote a seamless transition, this impedance is lessened for these students, thereby aiding in their academic success.

Beyond the initial transition period, researchers recommended that educational institutions pursue strategies including accommodations by the housing department (Robertson \& Ne'eman, 2008; Shmulsky et al., 2015), peer support groups (Dillon, 2007; Barnhart, 2016; Neville \& White, 2011), self-advocacy skills training (Adreon \& Durocher, 2007), structured social activities (Fox, 2011), video-self modeling 
interventions to enhance social-communicative skills (Mason et al., 2012), counseling interventions to enhance problem-solving skills (Pugliese \& White, 2013), and coordinated support through the school and outside rehabilitation agencies (Dillon, 2007). These recommended strategies were the result of research and observation conducted at the collegiate level, utilizing participants who were generally not identified demographically.

Neville and White (2011) recommended that the entire institution (e.g., faculty, advisors, therapists, disability services personnel, and others) should work in a collaborative fashion with the students identified with autism and their parents to ensure that the challenges faced by these students are collectively understood (Neville \& White, 2011). Brown and Coomes (2016) specifically recommended that institutions need to promote equity, advocate for universal design, acknowledge the uniqueness of each student identified with autism, educate the campus community, create and enforce supportive policies, facilitate proactive relationships with all stakeholders, and ensure effective accommodations are provided (Brown \& Coomes, 2016). To this end, researchers suggested that the institution's Disability Services Office should collaborate with the academic affairs and student activities departments to ensure that a variety of programs are offered to educate their employees and integrate the students identified with autism (Kelly \& Joseph, n.d.), or to train faculty and staff on effective methods of support and accommodations for students identified with autism (Peña \& Kocur, 2013).

The central thread of the recommendations by researchers for institutional approaches was that every aspect of the institution needed be fully involved in the development and implementation of mechanisms and strategies to aid in student success. 
The researcher contends that this approach should be the practice for all students, not just students identified with autism, and that by engaging a universal design as advised by Brown and Coomes (2016), the institution can actively assist every student in transitioning to and succeeding in a postsecondary setting.

Teacher factors. Paralleling the advice of Rendón, Kelley and Joseph (n.d.) posited that students identified with autism can benefit from teachers who build relationships beginning on the first day and reinforce these relationships through regular interaction. In addition, they suggested that these teachers should build relationships with the student's parents when possible and share their experiences of working with students identified with autism with others at their institution (Kelley and Joseph, n.d.). Gobbo and Shmulsky (2012) also focused on validation in that they advised that faculty can facilitate the success of students with autism by creating a classroom climate that encourages a diversity of expression, provides organization and structure, manages the emotional and environmental climate, and allows for individuality (Gobbo \& Shmulsky, 2012).

These researchers advised approaches that would validate and benefit every student; by embracing a relationship-based approach as well as classroom climate of diversity and inclusion, all students would benefit.

\section{Discussion}

Ever-increasing numbers of students identified with ASD are choosing to attempt a postsecondary education (Vanbergeijk et al., 2008). In doing so they transition from a regulated setting with parental involvement and institutional support to a postsecondary setting where decision-making, self-reliance and self-advocacy are expected. 
A review of the literature indicated that secondary school students with autism are likely to transition to a postsecondary educational environment if they possess the academic and verbal skills needed, a tolerant and caring attitude, and can advocate for themselves (Chiang et al., 2012; Giarelli et al., 2013). Additionally, the extent to which parents and family maintained a strong link to their child and served as a mentor can promote postsecondary success (Mitchell \& Beresford, 2014; Giarelli et al., 2013).

Research indicated that schools can foster successful transitions through providing formalized programs (Roberts, 2010; Wei et al., 2016), yet evidence indicated that students identified with autism may not be receiving such support due to a focus on other students (Levinson \& Ohler, 1998; Janiga \& Costenbader, 2002). Providing faculty and staff with the time and training to ensure effective instruction while building partnerships with parents (Chiang et al., 2012), instituting counselor-led programs to enhance student's social skills and executive functioning (Dipeolu et al., 2014), or ensuring rigorous IEP-driven transition programs (Szidon et al., 2015), may be proven effective for some but were unlikely to be sustained due to time and budgetary constraints; the most practical approach for addressing the needs of students with autism who are pursuing a collegiate path is to incorporate school-wide mechanisms and strategies that foster transition for all students, such as training faculty and staff to implement inclusive strategies or offering postsecondary introductory coursework on secondary campuses.

Once these students arrive at a postsecondary educational setting, they face the challenges of a new paradigm. The educational setting chosen may impede their success. They may possess the intellectual capacity needed for academic success, but their lack of 
independent living skills, sensory issues, self-advocacy variances or other socialization challenges may interfere with their acceptance within the postsecondary setting (Adreon \& Durocher, 2007; Prince-Hughes, 2002). This lack of acceptance can lead to feelings of isolation, anxiety and depression, which can affect academic performance and resilience (Sayman, 2015; Gelbar et al., 2014; Müller et al., 2008). Parents can mitigate these challenges by continuing to play a key role in the postsecondary experience through the providing of needed support and guidance (Ebel, 2015; Peña \& Kocur, 2013).

Research indicates that postsecondary institutions can implement a full range of processes and mechanisms to assist students identified with autism in this transition. The recommendation of a summer program prior to the freshman year (Kelley and Joseph, n.d.), and varying approaches to first-year support programs (Shmulsky et al., 2015; White et al., 2017) seem to be most promising in lessening the initial trauma of transition. The institutional recommendations of Neville and White (2011) and Brown and Coomes (2016) are most likely to ensure a uniform, equitable and inclusive approach to addressing the transition needs of all, including those identified with autism.

Individual classroom teachers at the postsecondary level can have a significant influence on the successful transition of students identified with autism. Teachers who build and reinforce relationships through regular validation of students and interaction with parents can enhance the success of these students (Kelley and Joseph, n.d.). Creating a positive classroom environment that welcomes diverse ways of learning can further aid students identified with autism in their transition (Gobbo \& Shmulsky, 2012).

Within the research, there existed a preliminary foundation addressing factors that influence transition both at the secondary school and within the postsecondary 
educational environment. Much of the research confirmed commonly held notions of practical approaches to addressing the needs of students with autism. This is not to say that the current state of research into transition is adequate; comparatively little research into this process has been conducted.

Research at the secondary school level has often placed a significant emphasis on the importance of the IEP and the transition plan. However, the existence of an IEP presupposes that students have been assessed and identified as within the Special Education domain; students pursuing a collegiate trajectory may be succeeding academically within the strictures of the secondary system, may never have been identified to the school, and are therefore not receiving specialized transition assistance.

Once a student begins their postsecondary experience, institutions expect that students will self-identify and self-advocate. Yet these students may lack the social skills and confidence to do so. The research articulated strategies to utilize with students identified with autism, but if those students are not identified, how can those strategies be utilized? Only by implementing mechanisms that ensure institution-wide inclusive instructional and administrative strategies can the transitional needs of all students, including those identified with autism, be addressed.

\section{Conclusion}

As students identified with autism increasingly choose a collegiate trajectory, researchers have sought to understand the factors that promote a successful transition for these students. Research addressing factors at the secondary school level generally resulted in findings that were of limited use; knowing that students with strong academic backgrounds succeeded in college only helps us identify those likely to succeed rather 
than assist all, and students with formalized IEP transition plans may be less likely to be pursuing a collegiate trajectory. Mechanisms for promoting a successful transition once enrolled in a postsecondary institution often revolve around strategies for overcoming symptoms concurrent with an individual's cognitive style, rather than embracing that diversity within an inclusive educational setting. The key to successful transition for students identified with autism, whether at the secondary or postsecondary level, is embracing a paradigm where the entire institution functions in concert to provide the most universally-designed, inclusive educational setting practicable, so that all students, including those identified with autism, can achieve to their potential.

This approach is efficacious in so far as it goes, but the strategy falls prey to its own definition; transition is a process, and as stated previously, secondary schools and postsecondary schools are two completely discrete and independent institutional settings. What currently transpires is not so much a transition as it is an end and a new beginning. This researcher posits that understanding this process of movement from an end to a beginning may hold the key to maximizing successful transition for students identified with autism.

Only a handful of researchers addressed how we counteract this abrupt process by either introducing aspects of the secondary school within a postsecondary setting (Zager \& Alpern, 2010), or providing summer programs for transitioning students (Kelley and Joseph, n. d.). While transition research is generally scant, this is where the true gap in research exists: viewing the movement from secondary to postsecondary school as a true transition that requires actions on the part of both the secondary and postsecondary 
schools to reach across and close the gap between institutions. This is where the work needs to be done. 


\section{Chapter 3: Methodology}

This qualitative study explored the experiences and perspectives of students identified with autism, their parents/caregivers, and other key parties as they transition from secondary school to community college. In researching transition, the researcher was stunned by data that indicated that nearly $86 \%$ of the subject population was reporting in high school that they intended to pursue a postsecondary education (Wagner, Newman, Cameto, Levine, \& Marder, 2007), but $68 \%$ of the same population was either not applying, not enrolling or not persisting in a postsecondary setting (Glennon 2001; Wei et al. 2012). This led the researcher to seek a better understanding of the transition experience as lived by students, parents and other involved parties to divine the critical factors impacting transition, with the intent of discovering an overarching mechanism that explained the transition process for students identified with autism.

This chapter (1) articulates the study's research questions, (2) addresses the reasons for utilizing a qualitative approach, (3) provides a detailed description of Grounded Theory as the chosen qualitative methodology, (4) discusses questions of ensuring quality research, (5) outlines practices relative to ethical considerations, and (6) describes the methods used in the collection and analysis of this study's data.

\section{Research Questions}

Two research questions guided this study:

1. What are the critical factors that influence college trajectory students identified with autism as they transition from a secondary school to a community college?

2. How do students with autism and other stakeholders experience this transition? 


\section{Reasons for Utilizing a Qualitative Approach}

A qualitative approach to research can be beneficial when considering fields of study that have yet to be thoroughly investigated (Creswell, 2003). As the topic of students identified with autism and their transition from secondary to postsecondary institutions has only recently begun to be researched, a qualitative methodology seemed most appropriate and was therefore selected. This methodology allowed the researcher to develop a detailed view of the transition process from the perspective of the participants. Utilizing the disparity between the stated intentions and actions of the students as a cornerstone of the inquiry, while remaining focused on a cohesive view of transition, required a more expansive consideration of the process; one that was not sufficiently represented by any one model of transition or human interaction. Developing a rich understanding of the factors that affect this disparity and inform the process required that a spectrum of participants be studied within their own domains and embracing their own perspectives. Qualitative methodology was best suited to manage this bricolage of structure.

Beyond the absence of a single encompassing model and the complexity of the participant field, qualitative methodology was appropriate for use in this setting in that it focused on the process as well as the product (Creswell, 2003). The researcher's intent was to capture the transition experience from the perspective of those transitioning and to create meaning from it to understand the disparity between intent and outcome and thereby divine the critical factors in play.

Finally, the researcher was aware that considering auspicious intents and inglorious results had the potential to be sensitive to the students as well as others 
intrinsic to the process. Qualitative methods facilitated the researcher in gathering descriptive and insightful data in a way that permitted each participant to "tell their own story in their own way", and thus added to the richness and authenticity of the findings in a considerate fashion.

\section{Qualitative Method}

Overview of Grounded Theory. Grounded Theory (GT) has as its origins in the work of Mead, Dewey and others, though it owes its name to Glaser and Strauss (Charmaz, 2014). Prior to The Discovery of Grounded Theory: Strategies for Qualitative Research (1967), qualitative research was being conducted, but much of the skill set necessary was acquired through field experience and mentoring; approaches were less systematic and more intuitive, thereby making it more difficult to effectively replicate. Glaser and Strauss were the first to codify GT. Subsequently, others could study and utilize their approach without the need to apprentice at their feet (Charmaz, 2014).

The work of Glaser and Strauss arrived at a fortuitous time in the development of research methods. The qualitative methods previously utilized in sociology were losing favor to more finely tuned quantitative approaches which, in the minds of some, were more palatable to scholarly journals, funding agencies and university departments. Positivist views of objectivity, generality and replication had gained favor; Glaser and Strauss challenged these approaches with GT. Moreover, they provided a systematic approach for conducting qualitative research which had its own logic and could generate theory (Charmaz, 2014).

GT as presented by Glaser and Strauss had seven defining components. These were: concurrent involvement in the collection and analysis of the data; constructing 
analytic codes and categories, not from preconceived theories, but from the data themselves; making constant comparisons during each stage of analysis; advancing theory development throughout the process of data collection and analysis; ongoing memo-writing as an aid in interacting with the data; conducting theoretical sampling to aid in theory construction; and conducting the literature review after the independent development of theory (Charmaz, 2014). Each of these components were further explored in the following.

Concurrent involvement in the collection and analysis of the data meant that the collection and analysis needed to coexist in a sort of synchronized dance; one analyzes data either while one collects it or immediately thereafter, depending on the demands of the situation. What resulted was a sort of interwoven tartan of collection and meaningconstruction. Through field notes the researcher was continually interacting with the collected data. When collecting data, the intent was to stay as true to the words of the participants as could be achieved while recognizing that once the words leave the participants mouths, they are changed by the context through which the words are heard by the researcher and the manner in which they were transcribed; raw data cannot ever be truly raw data. Regardless, analysis of the words was immediately begun to understand and make meaning from them; this was the ongoing collection and analysis step in the GT process (Charmaz, 2014).

When constructing analytic codes and categories, it was done from the data itself. A true Grounded Theorist does not enter upon research with a fully formed theory in their head that they wish to prove; it is rather the data that leads the researcher to the theory. As the Grounded Theorist moves through the data, progressing word-by-word, line-by- 
line, incident-by-incident, they capture the essence in terms of "words that reflect action" (Charmaz, 2014, p.116) and, to the extent possible, utilize the voice of the interviewees, if not their exact words. These are known as initial codes and represent the first step in the coding process (Charmaz, 2014.) These initial codes were subsequently analyzed to find the most frequent or significant codes to be utilized as focus codes, which offered a conceptual mechanism to facilitate processing large amounts of data into comparatively fewer classifications. Once focus codes have been extracted, they were further analyzed to distill even fewer, more encompassing classifications known as categories. By focusing solely on the data throughout the process, the researcher ensured that the resultant codes and categories are wholly derived from the data itself (Charmaz, 2014).

Making constant comparisons during each stage of analysis meant that at every step of the analytic process the researcher utilized comparison strategies to establish distinctions or trends. Initially, the researcher compared statements within a single interview or between interviewees. Discovering inconsistencies or commonalities can, as in the case of this study, lead to richer understandings of the data. When the opportunity existed to conduct longitudinal research, valuable insights were gained by comparing an interviewee's statements over time. Throughout the process, the use of constant comparison enhanced the researcher's understanding of the authentic nature of the experience as told by the data (Charmaz, 2014).

Within GT, theory development occurs throughout the data collection and analysis process. It is an ongoing process and a natural outgrowth of the interplay between data collection and data analysis. From the beginning of data collection, theories are built "from the ground up" (W. McIntyre Miller, personal communication, 
November 14, 2017); theories are posited, tinkered with, thrown out, retrieved, revived, amended and so forth through the analytic process to the conclusion of the study and/or publication of the results (Charmaz, 2014).

Memo-writing served several functions. It acted as the "pivotal intermediate step between data collection and writing drafts of papers" (Charmaz, 2014, p.162). Memowriting can result in a rich trove of transcribed thoughts that can be utilized in the draft writing process. Memo-writing also served to prompt the researcher to "analyze...data and codes early in the research process" (Charmaz, 2014, p. 162). Memo-writing was the researcher's opportunity to explore ideas and interact with the data in a documented fashion that could be referenced and further explored with later memos. Effective memowriting assisted in developing rigorous analysis and authentic codes, which led to sound theories (Charmaz, 2014).

Theoretical sampling was utilized to assist in developing saturated categories and rich theories. Theoretical sampling often came into play when the researcher had come to the point where tentative categories had been developed. It might have been that some categories seemed lacking in substantiation from the data, or that the data suggested a new, emergent category, or a data component was especially puzzling and out of place within the established categories. At this point, theoretical sampling was employed. The researcher either coded the data for an emergent category, which might have resulted in additional substantiation, or re-interviewed participants utilizing questions that addressed the shortfall or emergent category. The intent was to gather data to saturation, the point where new data is derivative. Theoretical sampling provides us with another strategy to enhance the richness of our analysis and theory development (Charmaz, 2014). 
Glaser and Strauss (1967) called for literature reviews to be conducted after the independent development of theory. Their thinking was that reviewing the literature early would predispose the researcher to see the data through the lens of the theories extant, what could be described as "received theory" (Charmaz, 2014, p. 306). The value of Glaser and Strauss' approach was that it served as an additional safeguard in favor of the data driving the theory. There is some debate over the degree to which this can be achieved, and compromises such as delaying the literature review until one is in the midst of analysis are often utilized (Charmaz, 2014).

While grounded theorists who have followed Glaser and Strauss have made changes in some aspects of their approaches to GT, their methodology still offered this study an effective, codified, practical approach for conducting qualitative research.

\section{Ensuring Quality Research}

Research findings can be subject to questions of analytic quality. It was reasonable and appropriate to inquire as to what criteria should be invoked and applied to evaluate this dissertation relative to analytic quality. In terms of quality, or rigor, within GT research, Birks and Mills (2011) presented a clear and concise explanation within Grounded Theory: A Practical Guide which was applicable within the scope of the proposed research.

The authors presented that though numerous factors influence the rigor of grounded theory research, these factors can generally be viewed in terms of the expertise of the researcher, the congruence of the methodology utilized throughout the process, and the precision by which processes, and procedures are conducted. 
Birks and Mills (2011) acknowledged that GT research could be somewhat overwhelming to the novice; even the professional researcher can find it challenging in their first foray into GT methodologies. They advised that leveraging generic skills and extensive reading within the field could mitigate the inexperience that the neophyte brings to the research. The review of Birks and Mills (2011) caused this researcher to recognize that his career conducting military intelligence research, though involving decidedly different topics and terminology, closely paralleled data collection, analysis, and theory formulation processes inherent in GT research. As such, the researcher had experience in a similar methodology which provided buttressing to the practices of GT research within this study.

Congruence within the methodology means that one's personal philosophical position, the methodological approach utilized, and the aims of the research as stated, are all aligned with one another. In terms of this study, this researcher acknowledged that transition had been woefully understudied and that to elicit the rich and authentic narratives needed to begin to understand this experience, a sensitive methodology that allowed for the drawing of meaning from the data was desirable. As such, GT research held the most promise for achieving these goals while aligning with the researcher's view that transition is a complex, nuanced experience and that to be understood, it must be approached from numerous perspectives within a unified, cohesive framework.

While precision within the conducting of research can be asserted by any researcher, the assertion is meaningless without the documentation to support such an assertion; in this regard, extensive and rich memo-writing was critical. Such memos provided the evidence necessary to prove that rigorous and consistent precision was 
utilized. In preparing for this dissertation, and while conducting the preliminary pilot study, the researcher utilized memo-writing both before and after data collection; perceptions were recorded prior to semi-structured interviews or other observations and compared with memos prepared immediately following the experiences. In doing so, the researcher explored the individual experiences of the participants as well as their interactions and the unified notions that became apparent to the researcher while ensuring an authentic and rich trail of precision research.

By leveraging the researcher's military research experience, ensuring that the various methodological components were aligned, and maintaining an exhaustive documentary evidence trail demonstrating precision GT research, the researcher intended to ensure that the research conducted was sound and of high quality.

\section{Ethical Considerations}

This dissertation was conducted under the auspices of Chapman University. Institutional Review Board (IRB) approval was obtained for both a pilot study and a more expansive study requiring both renewal and modification (Appendix A). Soliciting IRB approval required that detailed descriptions of research plans were presented for review, a process that was contrary to the emergent nature of grounded theory (Charmaz, 2014). This quandary necessitated that the IRB application be constructed in a manner that provided maximum flexibility within the study parameters. Still, the emergent nature of the methodology necessitated a subsequent modification of the initial study methods.

Special care was taken to ensure the comfort level of all participants throughout the process. This was accomplished by favoring comfort level over data collection and maintaining sensitivity as to when to probe for further information and when to refrain. 
Participants were fully informed of the voluntary nature of the study. No minors were participants in the study and all interviewees provided written consent prior to participating in the study (see Appendices B \& C, for consent forms). Participants were not compensated for their time and were advised that data and results presented in this dissertation might be used in part or fully for the researcher's other purposes.

\section{Participants}

Domain. The participating community college is located in north Orange County, California and was a convenience choice. West College (a pseudonym) has been in operation for over thirty years and currently addresses the educational needs of a diverse student population exceeding 17,000 . The feeder secondary school is in close proximity to the community college and is one of four high schools within its district. Its choice was again a matter of convenience. The participating secondary school has been in operation for over fifty years and serves the educational needs of a diverse population of nearly 2,500 students.

Study participants. The study called for a spectrum of participants to form a framework of understanding of the transition process. The pilot study utilized personal contacts, word of mouth, direct contact, and flyers provided to DSPS offices to identify three community college students, five parents and two community college disability services coordinators (one was retiring and the other replacing them) who were incorporated into the dissertation protocol. In addition, professional contacts at the secondary school were leveraged to obtain teacher and secondary school administrative participants. In turn, these teachers and administrators identified students to participate in the study. Further, participating secondary school teachers utilized their professional 
contacts to obtain community college teachers for the study. One parent who had been interviewed as part of the pilot study later asked that they and their child be removed from the study, which was done.

In all, twenty-six participants were interviewed or otherwise provided information for this study (Appendix D).

Confidentiality. To ensure the anonymity of the participants, and to maintain confidentiality of the data, processes were implemented in accordance with guidelines set forth in the IRB approval documentation.

The anonymity of all participants was guarded using pseudonyms assigned from a list of fifty randomly generated names provided by a web-based, random name generator (random-name-generator.info). These pseudonyms were used throughout the research, including in all documentation. For purposes of ensuring accurate communication with participants, a single list of pseudonyms and corresponding names was maintained in paper format and secured in a data safe when not in use. That document was destroyed at the conclusion of the research.

Confidentiality was maintained for all participants by (1) ensuring that all interviews were conducted in secure, comfortable settings that promoted confidential exchange, (2) recording all data on a secure handheld password-protected device, (3) securing the handheld device in a data safe when not in use, and (4) deleting all files once the analysis was completed.

\section{Data Collection Procedures}

Data collection began in October 2017 as a pilot study under the authority provided by IRB 1718H041. As a result of my dissertation proposal presentation, 
recommendations were presented that necessitated modifications to the initial IRB proposal, which were approved September 28, 2018. All interviews were conducted in accordance with provisions in place at the time of the interviews, and any changes that affected individual participants were addressed either by re-interviewing the individual or by excluding them from participation in the study.

Prior to conducting each interview, participants were informed of the nature of the study. If they found the terms of the study agreeable, participants were provided with the voluntary consent forms (Appendices B \& C). The form was discussed to ensure understanding, and if each participant still found it to be agreeable, they signed the consent form. This was followed for students by the Student Background Information form (Appendix E), which provided demographic data, including socioeconomic status and family composition. One student misrepresented themselves as being legally an adult and was discontinued from the study at this point. All other students completed the background information form. As appropriate, demographic data on other study participants was obtained during the course of the interviews.

After providing consent and demographic data, each participant was engaged by the researcher in a semi-structured interview utilizing an interview guide (see Appendix F). These interviews were conducted between October 2017 and October 2018, with the majority of the interviews taking place between April and October of 2018. The interviews were recorded using a smartphone application which tracked the date, time, and length of each interview. These interviews were conducted in person at mutually agreed to locations and were structured to last between 30 and 45 minutes. Secondary school administrators were interviewed in their offices at the high school campus during 
school hours. Secondary school teachers were interviewed either in their classroom after school or at a coffee shop a discreet distance from and outside the attendance area of the high school, to minimize the potential of encountering students. Secondary school students were interviewed initially off campus at a location of their choice, generally either a coffee shop or a fast-food establishment located in a shopping center proximate to the school. Community college administrators were interviewed in their campus offices. Professors were interviewed off-campus at a nearby coffee shop. The community college career technician was interviewed in the high school staff lounge. At all times, care was taken to ensure that the interview was private and that the participants felt comfortable to fully express themselves. On one occasion, an administrative interview was interrupted by staff, but it resumed shortly thereafter, and confidentiality was maintained. Subsequent interviews were conducted with participants as the emerging data warranted. These interviews were generally conducted at the same venue as the initial interview, with the exception of one administrator who had changed school sites and was interviewed via telephone. In the case of students, the research plan called for additional interviews during both the summer and fall time periods, and these were conducted as planned. Subsequent student interviews took place at mutually agreed to locations, including a public library, a restaurant, a park, and the participant's front porch. Students were also interviewed on the community college campus; this was intended as a sort of "shadow" interview to determine student reactions to the campus and areas of campus strengths and campus challenges (Appendix G). Of the thirty-six student interviews scheduled, family travel or other factors necessitated that the interviews be conducted on three occasions over the telephone. On four occasions, students requested 
that they be sent a subsequent interview to respond in writing. Given the need for sensitivity and the potential for these requests to be driven by anxiety or sociocommunicative variances, I complied. In addition, only three students agreed to allow the on-campus interview; two simply refused; another, after asking if they could "be honest", confided that they were not "comfortable" with the idea. In addition to the three, one other did provide responses to the questions intended for the "shadow" interview, though they did so in written format. As will be further explored, the other three were unavailable for the "shadow" interview.

In addition to the interviews, requests were made of the students for copies of the transition component of their IEP (if they had an IEP). Six students provided these documents; three reported that they had lost them or could not recall having received them.

In addition, all available institutional documentary evidence relative to transition was assessed for its relevancy and possible further analysis. This included the transition component of the IEP (Appendix J), the 2016-2017 School Profile (Appendix H), and the Spring 2018 school magazine article on student postsecondary destinations (Appendix I). The researcher recognized that some materials available through the schools were selfpromotional but evaluated it from that perspective and incorporated it into the research, thus providing added richness to the study.

\section{Data Analysis Procedures}

Data analysis began with the interview collection process; by conducting every interview himself, the researcher was able to assess each individual's responses not simply from their words, but also from their tone, body language and demeanor. 
Additionally, these assessments were conducted through the lens and subjectivities of a single individual, resulting in a more uniform and cohesive assessment. These observations provided valuable insight into each participant and were recorded utilizing the smartphone application as memos immediately following the interviews.

Further, each interview and memo were transcribed by the researcher personally. After transcription, the written interviews and memo were compared to the recordings to confirm the fidelity of the written record and to mine for meaning. In this way, each participant interview existed in both a "raw" and "polished" version. The "polished" version was mildly de-naturalized to the extent that it aided in understanding intent without losing meaning. The final step in the transcription process was to email the transcriptions to the participants to confirm that their intent was accurately reflected. Of the twenty-six participants, 17 responded to the emails. Of those, 14 indicated that they recommended no changes to the transcriptions. Of the three who did recommend changes, no changes were substantive, and all were implemented. During the transcription and member-checking process, additional insights were perceived and included in the transcribed memos.

Both conducting and transcribing the interviews allowed the researcher to remain concurrently involved in the collection and analysis of the data; this resulted in a synchronization of activity, leading to a fine weaving of collection and meaningconstruction that remained true to the words of the participants while developing a depth of understanding. Throughout the process, memo-writing was utilized to collect ideas, musings and observations relative to the participants, the environment, and the study. As an analytical tool, memos were used as a mechanism for exploring concepts and ideas 
and interacting with the data in a documented fashion that was accessed for the writing process.

Constant comparison throughout the analysis process, beginning with the two DSPS Coordinators, led to discoveries that resulted in a richer understanding of the data. The DSPS Coordinators provided a triangulated understanding of the culture and architecture of the office from two different perspectives; one coordinator was literally days from retirement and had an involvement with services for "disabled" students going back to 1976 and the Lanterman Act, while the other was comparatively young and knowledgeable, but inexperienced.

As was the case with the DSPS Coordinators, comparing within the same category of participant provided an opportunity to develop a richer understanding of the experiences of those participants; in the case of the students, the multiple interviews over a six-month period allowed not only for comparison between participants but also for a longitudinal approach. This provided valuable insight into student struggles and possible reasons why students fail to persist in their transition. These findings are presented in Chapter 4.

The transcription of interviews and memos resulted in 47 interviews and 26 participant-affiliated memos. The interviews encompassed over sixteen hours of recordings and the memos utilized 14,364 words. Utilizing the qualitative data analysis software program known as NVivo 12, these documents were initially analyzed using line-by-line, or as appropriate, incident-by-incident coding to capture, in terms of action, the essence of the voice of the participants relative to their experiences involving transition from secondary school to community college. The amount of time involved in 
this process was uncertain but prodigious and resulted in 326 initial codes representing 1329 separate initial-coded incidents.

For the second phase of coding, the researcher revisited the initial codes to discern those codes which appeared more frequently or appeared to have more significance. This process involved assessing the initial codes and determining which functioned best as mechanisms for categorizing the data fully, in a way that sharpened and highlighted the emerging data. Part of this process involved returning regularly to the memos for each participant as well as the timeline-memo to seek clarity and perhaps a degree of inspiration. The commonalities of experience and repeated references within the participant interviews led the researcher to distill the initial codes into 60 focused codes.

It was during this secondary stage that the researcher became self-aware that there was more than a little subjectivity to the process of analysis. One has to look at the data and perceive commonalities, determine significance, and obtain inspiration; as such, structuring the analysis was as much art as it was science. If a common experience was repeated several times in the interviews of a group of participants, that repetition could be counted mathematically. As a result, experiences that were often repeated could be perceived as more important and were accordingly assigned greater significance. However, what of a single word or phrase that appeared once but seemed to have profound impact? Assigning significance to that moment was a matter of artistry and subjectivity.

Data analysis within the Grounded Theory realm is not a matter of divine inspiration; nor is it simply a matter of, as Michelangelo asserted, removing the excess rock to reveal the sculpture. As experienced by this researcher, data analysis was as 
much a matter of trial and error as artistry. Perhaps it was that the researcher is a visual learner; moving the individual codes about within the framework provided by NVivo 12 allowed for viewing the data in multiple arrangements to determine what seemed to make the most sense.

The sixty focused codes were further analyzed, using a sort of reverse axial coding process. Rather than building around the axis of a category, intense scrutiny of the focus codes, considering multiple possible arrangements and occasionally discarding "excess rock", resulted in an emergence of commonalities which guided the researcher to the axis: the eleven categories that defined the emergent factors, conditions and cohesive theory.

These eleven emergent categories are (1) autism particulars, (2) community college promoters of transition, (3) community college roadblocks to transition, (4) high school promoters of transition, (5) high school roadblocks to transition, (6) internal struggles, (7) other, (8) personal support from a familiar, (9) pre-high school factors, (10) structure, and (11) summer roadblocks. These appear in the order in which they developed within the NVivo 12 mechanism; not in relative importance.

Further review led to a consolidation of several of these categories resulting in five themes of transition for students identified with autism. These were: (1) secondary school promoters and inhibitors to transition, (2) the critical nature of the summer "gap", (3) community college promoters and inhibitors to transition, (4) student struggles and affirmations, and (5) the essential support of a "familiar".

As the researcher pressed forward toward the emergent categories, he returned to the data to utilize theoretical sampling for confirmation and support, which the data 
provided. He continued to return to the data, actively seeking discrepant information that might contradict the emergent theory; little of significance appeared. Ultimately, the categories were confirmed, and saturation was ensured.

Data analysis within the Grounded Theory domain is not an expeditious one; no record exists of the time involved in this process, nor would such a record be of use. GT analysis is a sort of reflective inquiry whereby one considers the data and tries to make sense of it. Much of what takes place within the data analysis component takes place within the mind; a realm where time is not standardized. NVivo 12 served as a tool to organize and experiment with ideas that emerged first cognitively.

Certainly, throughout the analysis, the seven defining components of Grounded Theory as set forth by Glaser and Strauss (1967), and the guidance of Birks and Mills (2011) relative to rigor, guided the researcher; data analysis is an arduous and uncertain terrain. Using their guidance to leverage a rigorous interplay of data collection and analysis, in concert with interaction with the data and cognitive rigor, resulted in the emergence of a theory grounded in the data. The findings that emerged from this data analysis are presented in Chapter 4.

\section{Summary}

This study utilized Grounded Theory to explore the experiences and perspectives of students identified with autism, their parents/caregivers, faculty/staff and other key engaged parties as they transitioned from secondary school to community college. In this chapter the researcher articulated the study's research questions, addressed the reasons for utilizing a qualitative approach and provided a detailed description of Grounded Theory as the chosen qualitative methodology. Additionally, the researcher discussed 
questions of ensuring quality research, outlined practices relative to ethical considerations, and described the domain where the study takes place. Finally, the researcher outlined the processes relative to the recruitment and confidentiality of participants, as well as the methods used in the collection and analysis of this study's data. 


\section{Chapter 4: Findings}

\section{Introduction}

This dissertation explored the experiences and perspectives of students identified with autism, their parents/caregivers, faculty/staff and other key parties as they transitioned from secondary school to community college. The findings provided a preliminary understanding of the myriad of mechanisms that impact transition, and indications as to why so few who espouse an intent to pursue postsecondary education engage or persist in that endeavor. Further, the findings pointed to factors that can be leveraged to promote a successful transition for students identified with autism.

To review, the researcher conducted 47 interviews with 26 participants and utilized Grounded Theory (GT) in the collection and analysis process. Following the GT principles set forth by Glaser and Strauss (1967), and the guidance of Birks and Mills (2011) relative to rigor, the researcher engaged with the data in both an artistic and a scientific manner to "coax" the emergence of codes, which ultimately led to overarching themes and theory grounded in the data. These themes centered on (1) secondary school promoters and inhibitors to transition, (2) the critical nature of the summer "gap", (3) community college promoters and inhibitors to transition, (4) student struggles and affirmations, and (5) the essential support of a "familiar". These overarching themes as well as emergent sub-themes are illustrated using evidence from the gathered data.

\section{Secondary School Architecture and Culture}

Each secondary school has its own unique architecture and culture derived from its history and traditions, views and attitudes of staff, student perceptions, and the geography of the school environment. In the case of the participating secondary school, 
the architecture and culture present resulted in attributes that both promote and inhibit successful transition for students identified with autism. Transition is promoted at the secondary school through their culture of celebrating postsecondary attainment and the presence of a robust, targeted, programmatic architecture. In contrast, transition is inhibited by the high school through a tradition of focusing on four-year acceptance versus community college, a high reliance on individual teachers to implement the transition program and consequential susceptibility to failure, and a significant service variance for students identified with autism dependent on their special education classification. The findings have the potential to effectively support or significantly impede the transition of students identified with autism.

Celebrating Postsecondary Attainment. The secondary school under study evidenced a culture of celebrating postsecondary attainment. Such celebration creates an environment where attending college is perceived by the students as a positive to be pursued, thereby promoting transition.

Public domain, district-published documents suggest that the school celebrates postsecondary attainment. The 2016-2017 School Profile (Appendix H, redacted for anonymity), provided a picture of the participating secondary school as focused on Advanced Placement courses, AP and SAT scores, and colleges/universities attended by recent graduates. Over half the document addressed these concerns. Also, the school news magazine published in its final edition of the 2017-18 school year a list of the graduating seniors and the postsecondary school that had accepted them (Appendix I). These documents highlighted a celebration of postsecondary attainment that was reflected in the views of two of the nine students. Kim stated: 
It (graduation) is a huge relief, and another reason being because all of my good friends are in college and then the college I'm going to be going to, they go to that same college, so I'll be reunited with them once again and not just hanging out outside on weekends.

Chris stated:

I'm feeling a bit nervous (about graduation) because after this, like all my friends, I go to college and figure out what I actually want to do as a career, and hopefully I'll figure it out at the JC I'm going to...”.

Secondary school administrator Catherine Green illuminated the school's culture most succinctly:

We like to think of this school as a reflection of the community. This school is in the center of one of the wealthiest communities in Orange County. The parents typically have multiple college degrees and expect the same from their children. We are here to provide that opportunity. Our goal, though we know it cannot be completely achieved, is to have all our students transition to a postsecondary school.

Students identified with autism who attend the subject secondary school are educated in an environment that celebrates postsecondary attainment. As such, they find themselves surrounded with individuals who perceive a postsecondary education as the expected norm. In the same way a family of teachers or police officers may, by their enveloping presence, promote their young to pursue the "family business", students who attend this secondary school are enveloped with the expectation of transition to college and may find themselves choosing this expected course of action. 
Robust Programmatic Architecture. The secondary school has in place a robust structure that promotes transition to college. Through the architecture in place, students have multiple opportunities to focus on their postsecondary intents and link with the community college as means of pursuing those intents. This defined approach increases the likelihood that students will connect with the community college and initiate the transition process.

Transition to a postsecondary setting begins comparatively early for students identified with autism who have an active IEP. According to Case Manager Lois Perry: As they are going through high school - when they become 16 we're obligated by the IEP process to develop an individualized transition plan - if they're $15^{1 / 2}$ and by the time you have the next IEP they'll be $16^{1 / 2}$, you have to develop it then, if they're $14 \frac{1}{2}$ and they'll be $15^{1 / 2}$ by the next IEP you don't have to develop until the following year.

The particulars addressed in the transition component of the IEP are delineated by Case Manager Frances Bennett:

Well, according to federal law, during their IEP which happens, which would be in place during the time that they turn 16 , they have to have a transition plan in place that allows for their education plans for after high school, it's education, it's employment and it's independent living.

Secondary school counselor Stephanie Taylor provides her sense of the timeline progression in stating, "It (transition) gets discussed briefly in ninth and tenth grade and a lot more seriously at eleventh and twelfth." 
For all students, including those identified with autism who may not have an IEP, transition mechanisms begin in earnest in the fall of their senior year. English teacher Doris Hall explained:

In the fall, we actually have representatives from West College as well as East Community College District come talk to the kids; all the seniors go during their English class. They go over different requirements, how to register, all that stuff. Someone from West College actually comes at least once a week; I believe it's Thursdays during lunch in our library to help assist. Also, this just started, we have a representative coming three days a week to provide assistance with career selection and job research - if you want to do this, where you go, what you do. That's all paid for through the community college. There're also field trips for the kids to go to West College to go see the campus as well. We also do the placement testing here for Math and English placement just because they are more comfortable here; they don't have to go over summer.

Outreach technician Barbara Wood clarifies her role in transition:

So, in my role as a West College rep... I work with students specifically for career pathways, so I give presentations to students explaining my educational background, giving myself credibility of course, and then I bring it into different career pathways, different certificates or different programs that they could get involved in, degrees that they could earn at West College, and if they want to move on to a four-year we can explore that as well. After my presentation, I typically have my laptop with me, and I will ask them if they want to meet one- 
on-one. I am happy to talk with them about different careers...My main focus is on careers and advising them about different options.

In addition, English teacher Doris Hall relates that other mechanisms exist to ease students into community college:

Actually, my zero-period class is technically a college class. We do bridge classes here on our campus. It is just a regular English class, it shows up the same on their transcripts...it allows them to get the college experience in a more comfortable environment to start out with sooner.

Through the Individualized Transition Plan (ITP) mechanism for students with IEPs, fall assemblies for seniors, classroom outreach, on-site community college counselors, college courses offered on the secondary school campus and field trips to the community college, the secondary school provides a robust, structured architecture promoting transition to the subject community college. These structures provide multiple opportunities for students to engage with the community college as a mechanism for pursuing postsecondary goals. The ongoing presence of the outreach technician and community college counselor provide further opportunities for engagement with these individuals, which would further promote enrollment, thereby initiating transition.

A Tradition of Focus on Four-Year Institutions. While the secondary school celebrates postsecondary attainment, they focus on four-year institutions as the preferred postsecondary goal. Students experience this focus as subtle pressure on the part of teachers, and teachers acknowledged awareness of the stigma attached to community college. This emphasis on four-year institutions creates an expectation that may be difficult for some students to meet. The reaction to this expectation by students can be 
profound; students reported acceptance to four-year institutions, though they were not actually accepted. This "faux" acceptance may be a coping mechanism for addressing the pressure to enroll in four-year institutions and lead to students deferring actual transition action in favor of perception.

The same public domain district published documents that demonstrated a celebration of postsecondary attainment also illustrated a focus on four-year institutions. The 2016-2017 School Profile (Appendix H, redacted for anonymity), made no reference to community colleges whatsoever, although they listed 66 "Universities of Recent Graduates". The school news magazine (Appendix I) listing of students and their postsecondary schools did list community colleges, and a visual assessment indicated that the plurality of graduating students chose community college as their postsecondary destination. However, the page layout emphasized four-year choices by placing prestigious schools at the top and sides of the pages and encapsulating the community colleges at the bottom-left-center. A continental map of the United States overlaid and angled towards the upper right further directed the reader away from the community college list. This focus on four-year institutions is supported by Briana who stated: In getting ready for graduation, there was a big influence on going to a four-year college. The teachers talking about where they went and pushed you to think about going there. Also, you know, we had pep rallies about college and academic success, and it seemed like there was a great value placed on the gradepoint average and getting into a four-year college. 
This view was supported by English teacher Doris Hall:

When I started here, community college was a bad word. Nobody wanted to admit that that was where they were going. That dynamic was challenged by the recession (of 2008). In the midst of the recession that (community college) became a reality for a number the students, but the stigma is still present here. The emphasis on four-year institutions was evidenced in the account provided by RSP teacher Joe Rodriguez:

Last year I had two special ed kids who had a GPA of probably, maybe a 1.8, so they are D, maybe D+ students their entire life. These two students were bragging - one was going to Yale; the other was going to Harvard. They both apparently had already been accepted - this was before you could actually legitimately be accepted - and they were arguing and bragging about which one was better about which college they were going to. In other words, these kids see all the attention we give to the students who are headed for University, and they want to be a part of that, even though there is no way they'd last a day there.

Emphasis on four-year institutions versus community college places pressure on students identified with autism to pursue a course of action that may not be in keeping with their best interests; research indicated that these students may be more successful if they live at home for the first year, thereby avoiding residential upheaval while addressing the change in academic venue (Perner; 2002). Given that within this study setting the closest public four-year institution is more distant than the community college, attendance at that college would require either significant transportation mechanisms or 
living away from home, either of which would likely add anxiety to and further complicate transition.

Beyond the students who might pursue a four-year institution in spite of the roadblocks, the secondary school's focus on these institutions creates an unrealistic expectation for many students; as indicated by Joe Rodriguez, this unrealistic expectation may result in students deferring all action in favor of "faux" acceptance, a coping mechanism that may result in no legitimate transition action being taken. Whether these students simply deferred or completely abandoned legitimate transition action in favor of social perception, the result of this behavior may be that if the student later chose to enroll in community college, they may have missed opportunities for beneficial services such as preferential enrollment or on-site visits. Missing these opportunities can complicate transition and increase anxiety, resulting in a lowered likelihood of successful transition.

Reliance on Individual Teachers. Though the secondary school has a robust architecture that promotes transition, this architecture was heavily reliant on the individual efforts of teachers and could be negatively impacted or completely circumvented by the actions of individual teachers as well. In the case of students with IEPs, their approach to transition is memorialized within an Individual Transition Plan (ITP). The development and implementation of legitimate and useful ITPs are reliant on the individual case managers; these managers are under significant pressure to balance their need to be student advocates with the need to prepare IEPs that are resistant to litigation. This can result in documents that are "correct" rather than useful. 
Multiple opportunities were available to students during their senior year to develop an understanding of the services offered through the local community college. In spite of this, the actions or inactions of individual teachers can and do preclude whole classes of students from obtaining this understanding.

Together, these findings can result in ITPs that are of little use to the student, and a robust, programmatic architecture that may not reach the students; students may leave high school with little guidance or understanding relative to their post-high school options, thereby increasing the likelihood of a haphazard transition.

Reliance on case managers for useful ITPs. For students identified with autism who have an active IEP, the ITP is intended to serve as a navigational device, leading the student to a successful transition. However, Case Manager Frances Bennett indicated that absent effective advocacy, the ITP may not provide the intended navigation:

It's tough because at the end of the day we are given these computer forms on which we are required to write an IEP. These forms provide very little wiggle room. We have to go with, on the ITP, go with whatever the kid says no matter how outlandish it is... "After graduation from high school I will..." I have to write down whatever the kid says. And that can lead to some pro-forma terminology... I try to advocate for a realistic ITP, but sometimes it's very difficult...

The views of Frances Bennett were echoed by the ITPs obtained from the students. Appendix $\mathrm{J}$, (names redacted) provided a clear illustration of the melding of districtmandated language "Upon completion of school I will" and the spontaneous comments of 
students "look into going to a Jr. College", "be a fashion designer". Ms. Bennett elaborated on the challenges of devising a useful transition document:

I want what's best for the students, and I think that gets lost sometimes in the dayto-day minutia of making sure that we're obeying federal law, and keeping the district out of trouble, and keeping ourselves out of trouble while still servicing the needs of the student ... As far as transition is concerned, we want to make sure we have realistic, attainable goals in place that really help the students, but we have to abide by district, state and federal regulations and procedures and make sure we don't write something into the ITP that results in litigation. Most of us do the best we can in an impossible situation. For the ITP, sometimes it's just best to use standard transition verbiage from the SEIS (Special Education Information System) Goal Bank. These goals may not be especially specific to the individual student, but they meet the requirements and are legally defensible. Transition mechanisms and the role of teachers in their efficacy. Mechanisms for a successful transition begin in the senior year with the fall assemblies. While this was adopted as a programmatic mainstay of the transition architecture, RSP teacher Joe Rodriguez highlighted the role an individual teacher plays and in so doing, illustrated the impact of reliance on individual staff actions:

The other thing that I - I kind of just initiated it and through many, many years now developed the responsibility (laughter) you could say (more laughter), it's not like, as if someone told me to, it's just something that fell into my lap. In the scope of doing it, as a case carrier, I networked with some of the local colleges around here to develop some relationships and so over the years I coordinate, and 
I do this for all the graduating seniors at the school, so I tell all the case carriers, I say "Hey, everybody who wants to go to West College or East College or whatever"...we do all that here on site.

The role played by Mr. Rodriguez was echoed by Case Manager Lois Perry: Yes, yes, yes. One of our case managers arranges for West College, and I think we also get information from East College. Our case carrier that has been inviting West College to come over and do the presentations and do the testing and everything has been largely successful, and we've been using it each and every year for, gosh as long as I can remember, so it has to be for at least the last 6-8 years, since he's been here and he took it over, organizing that transition for 12thgraders from another teacher and he's done a great job with that. As significant a role as Mr. Rodriguez played in facilitating mechanisms that promote transition, other individual educators may act in a manner that inhibits access to transition services. English teacher Doris Hall highlighted the challenges to the transition program that individual teachers can represent:

All seniors go (to the fall community college assemblies) with their English teachers. So, I take my senior English class. I take my class, but I do know that there are others in the (English) department that value their instructional time to the point of not taking their classes to the assemblies. They see college as something the students should do on their own, not as part of the instructional day. Then there are others in the department that will send their students but not go themselves - what kind of a message does that send? 
The challenge of accessing classroom time was related by Outreach Technician Barbara Wood:

Well, one of the persons that I work with has helped me get into some of the social studies classes, which is really great because he has the connection with those teachers, so that was kind of set up, you know - an email was sent out before I got here letting teachers know that I would have the service available to them, "this person is coming and she would like to give presentations". Unfortunately, not all teachers read their emails or want to give up class time for a presentation and so I've been connecting with the counselors a little bit, speaking with some of the counselors and letting them know that I'm available and some counselors have more connections with some of the students and staff members, so I was able to get into some classrooms that way as well, by going that route as well.

These findings portrayed a school environment where, in spite of a robust transition architecture, individual teachers can and do significantly impact the efficacy of the design. Individual case managers find themselves in the difficult situation of advocating for their students while conducting themselves in accordance with law and administrative directives; utilizing standardized language provided by the SEIS goal bank does much to ensure a defensible ITP but may not provide legitimate guidance for the student in their efforts to understand and plan for life after high school.

While multiple opportunities were available to inform seniors of the services offered by the community college, there was an understanding among the secondary school staff that the individual classroom teacher functions as a sort of "gatekeeper" for 
their students; if the teacher views the assembly or classroom presentation as a poor use of instructional minutes, or too much of a bother, or simply forgets to read their email regarding these services, the students were denied the informational opportunity.

By designing ITPs that served little use as guides for the student, or by denying students access to information about community colleges, the robust transition architecture in place at the secondary school was rendered ineffective. While the number of students affected by these actions was uncertain, it is probable that some students identified with autism were impacted, thereby affecting their transition guidance or opportunities.

Service Variances. Within the secondary school setting, students identified with autism may be provided with a range of transition services depending on how they have been identified and to what "lane of services" they may have been assigned. Students may be provided with an IEP, opening access to a range of accommodations and supports, they may be identified with a 504 and receive minimal accommodation and no formalized support, or they may be perceived to "have autism" but may not have been formally identified within the school community. Depending on the vagaries of the identification process, a student might find themselves in one lane of service or another. As the determination as to whether a student is provided with an IEP or a 504 often results in significantly different degrees of services and accommodations, similar students might receive significantly different services, thereby impacting readiness for transition and transition itself. As secondary school counselor Stephanie Taylor states:

A student with mild autism may or may not have an IEP, depending on whether the parents have pursued a medical diagnosis and whether they have pursued an 
IEP through the district. If they have an IEP, then transition planning is part of that IEP starting usually in tenth grade. If not, they might have a 504, which informs teachers of the student's needs but doesn't provide the same supports. Case manager Frances Bennett related that the need for an IEP is typically based on academic performance:

Would a student with high functioning autism have an IEP? Well, that kind of depends. There are students who have autism that, while it may affect them in their social lives, it doesn't necessarily affect them in their academic life. If a student can go into the Gen Ed classroom, can perform at the Gen Ed level, can complete the Gen Ed work, there's no reason why they would need to have an IEP.

Frances Bennett related that there may be students who would qualify as having autism but have not been identified to the secondary school:

We have a student right now who is - there's no doubt, the English teacher mentioned to me, there is no doubt that this kid has autism, but he does not have a 504, he does not have an IEP; he is enrolled in and succeeding in the general education class. If that is the case, we are not going to get in his way. If he is able to perform the work and do it without any accommodations or modifications, we are not going to interfere with that.

Bernadette Foster related how her daughter was diagnosed in elementary school:

We took her to a psychiatrist; actually, to my counselor that I was going to for counseling with the girls. She said she would do a screening and then refer her as necessary for diagnosis. She was diagnosed with very, very high functioning 
possible Asperger's and ADHD, and we got her on medicine... but her main issue at that time was that she not able to play with other children; she was unable to sit for more than ten minutes in a group setting and she didn't make peer friends very well. She did not interact well with the other children.

The diagnosis of Asperger's and the reliance on medication led to a situation in which the daughter was unaware of her autism and the secondary school was uninformed. As stated by her stepmother Claire Williams:

I'm not aware that she had any transition services, simply because she did not know what her diagnosis was, so realistically any person approaching her from the Special Ed department would've been completely out of the ordinary for her and would have been something for her to question.

Analysis of the data presented a picture best described by the words of Frances Bennett, "Well, that kind of depends." The actions of parents, medical professionals, individual referring teachers and the student study team may result in a student being identified as "having autism" and possibly resulting in an IEP. These same stakeholders may not perceive the needs of the student as substantial. They may simply request and receive a 504 designation, resulting in no services and receiving accommodations as the individual teacher sees fit. Conceivably, a similar student could go unidentified by the school, be scheduled into general education classrooms, and if no teacher refers the student to an SST, they might be known to the teachers as effectively "having autism" but receive no specialized transition services.

The range of services based on student classification creates an interesting dynamic; some students receive significant transition services and others may not, while 
their desire to enroll in community college and their potential to persist may be commensurate. Through this approach, "students identified with autism" could receive significantly discrete and disparate services and accommodations relative to transition, thereby potentially affecting successful transition for some students.

\section{The Challenge of the Summer "Gap"}

Students graduate from high school and find themselves in a sort of "holding pattern" for three months; graduation completes the official responsibilities of the secondary school. Community colleges may begin their association with a transitioning student through enrolling them, but legitimate responsibility begins with the first day of classes. This creates a twelve-week void where neither the secondary school nor the community college have significant influence. This period is experienced in two distinct ways; secondary school acknowledges that the cocoon of services and accommodations previously provided ends abruptly, and students experience this gap through a variety of unstructured and other-than-college-focused activities.

\section{"we wave goodbye and they are magically cured"- The End of Secondary}

School. The conclusion of secondary school as punctuated by graduation results in an official end to the support mechanisms present in K-12 public education. As Case Manager Frances Bennett states:

The tough part about transition per se within the high school purview, and it's referred to privately among the teachers, is that as of June 15th, 16th, whatever the last day of school is graduation for a senior in high school, we lead them out, we wave goodbye and they are magically cured of their disability. All of these accommodations that they are given through all 12 years of schooling, go 
away...You know, so like I said, it has always been gallows humor that the students become magically cured after high school.

Secondary School administrator Catherine Green expressed concern over the finality of the process:

We recognize that graduation can be abrupt for some of our population. We have such a great population and a big population going over there (to the subject community college) that we need to do a better job of bridging that gap. It's not just, "here, we're done", although we have had them for 12 years, you know we have to be able to bridge that gap a little bit.

"playing Xbox with my friends" - The Unstructured Nature of the Gap. The

period between the end of one school year and the beginning of the next, generally referred to as "summer vacation", is often utilized by students as a time of relaxation and a respite from academic endeavors. In the case of this study, the three-month period spanning graduation from secondary school to start of classes at community college was characterized by four of the nine student participants as a time that can revolve around unstructured activities or points of focus not directed towards postsecondary transition. Raymond spoke of his summer plans as, "playing Xbox with my friends". Amy related that she was "going to spend the summer helping my aunt with the homeless at the church". Martin stated, "My plans are just to finish high school and have a little celebration. I'm going to go to Chicago, meeting my cousins for a few weeks, and then when fall comes I'll be going to West College for a semester." Kim shared, "I've been doing a lot of sleeping in and relaxing”. 
The summer "gap" presents the challenge of navigating this void and arriving at the first day of class prepared to continue academic studies. Secondary school staff understand this challenge, expressing concern over the loss of services and lack of bridging provided. Implicit in the data was the secondary school's realization that their mandate limited what could be done after graduation.

Students reported participation in a number of unstructured activities unrelated to college attendance. Students saw this time as a period of relaxation and distraction; only one student mentioned a return to college; others did not express a focus in that direction. The danger inherent in this distraction is that students may not regain focus after summer; although all nine student participants reported in their initial spring interviews that they intended to attend community college in the fall, three of the nine failed to attend during the fall, with one reporting that they would enroll in the spring. The impact of the summer "gap" is critical in that it disrupts the transition with a void of unstructured distraction. In doing so, it provides an opportunity for students to reassess their transition plans or simply disregard them, thus leading to a breakdown in the transition process.

\section{Community College Architecture and Culture}

Every community college has its own distinct architecture and culture derived of its origins, faculty and staff attitudes and perceptions, student views and experiences, and the school environment. The architecture and culture of the community college under study displayed attributes that both promote and inhibit a successful transition and ongoing persistence for students identified with autism. The community college promotes transition through the fostering of a nurturing environment, geographic proximity to the feeder secondary school and a robust program of outreach. Their efforts 
at aiding transition are harmed by outreach that is reliant upon grant funding, a lack of understanding within the Disabled Students Programs and Services (DSPS) office as to the specific needs of students identified with autism, and faculty that can be either unreceptive to or untrained in the services and accommodations available for these students.

Nurturing Environment. Community college staff report a desire to provide a warm and welcoming environment for students identified with autism and the students reported perceiving the community college as a nurturing place. Providing a nurturing climate is one way in which the institution affirms presence, thereby promoting student persistence. Ensuring a climate that welcomes students was a consideration of Dr. Robert Miller, DSPS Coordinator who stated:

You know, it's hard to know for sure, but just being a warm and welcoming community, and I think we are pretty well-known as a warm and welcoming place for all students and that includes our disabled students. Certainly, we do the best we can.

Dr. Miller's comments were echoed by community college teacher Terry Edwards: Autism, huh? I see. My role in transitioning all students, including those with autism, is to assure that my classroom is a welcoming environment where all students feel comfortable, where they understand the expectations, and where they can succeed without too much stress.

These efforts at providing a warm and welcoming environment were reflected in positive perceptions expressed by four of the nine students. Briana stated, "I honestly feel very supported at school", Amy related, "I think they do everything they can and do 
pretty much everything great", and Martin exclaimed, "Everything there is, is perfect." Kim expanded on the topic:

Honestly, I think the college values me. I really see that it's just a warm, loving environment. From what I can tell everybody is so nice over there. It's way better than high school. In high school it's like the usual intimidation you get is like, "College is going to be so much harder, they're not gonna help you at all!" But like in college they're helping you every step of the way, especially your first year and it's, it's kind of like a family over there.

These four students experienced a degree of comfort within the community college environment commensurate with the intents expressed by the professional staff. While it was unclear what exactly drove these four students to perceive the community college as a nurturing environment, a review of the demographics outlined in Appendix D indicated that these students shared the common thread of parents who have college experience. It may be that the familiarity their parents have with the college environment resulted in a greater awareness and lessened anxiety towards postsecondary education, and a predisposition to view college in a positive light. Coupled with a welcoming environment, this likely encouraged these students to express positive views relative to the community college and those views likely translated to an increased likelihood of collegiate persistence.

Geographic Proximity. The closeness of the community college to home was mentioned by four of the nine students. Students viewed the closeness as either a selfevident positive or a positive in terms of being able to live at home while attending school. The views of these students were supported by the secondary school counselor 
who viewed local college enrollment as typical for students with autism, so they could adjust to the academic and social changes without having to concurrently adjust to a residential change.

Steven, when asked what he liked about community college, said "I like it because it's close by." This was echoed by Martin who stated, "Yeah, ...I decided to go to West College because it's the closest to my house" and Kim who related, "The college is really close to my house. I'd say about ten or fifteen minutes, maybe, maybe less than that." George didn't address time or distances, but said, "It's important to me that I live at home when I attend college". Living at home was echoed by secondary school counselor Stephanie Taylor who stated:

Generally, we'll find that they go to a local school because that idea of them needing to live in the dorm setting is not appropriate for them. Being away from home, out of their routine, stuck with strangers all of a sudden is not the right thing for them, and so we find that they will apply to very local schools so that they still can maintain residence with mom and dad.

While these students expressed geographic closeness to the community college as a benefit, their underlying reasons were somewhat different. Steven and Martin seemed to express that proximity drove the choice of community college, whereas Kim commented on the distance to college more as a point of interest than as a deciding factor. George was less focused on the proximity to school than the proximity of the school to home.

Though each had their own perspective, the students were consistent in viewing closeness of the college to home as a positive. Stephanie Taylor echoed this view in her 
comments about why students with autism typically chose local postsecondary institutions; her views reflected the views of previous researchers, who recommended local college attendance to provide an opportunity to adjust to academic and social changes without having to adjust to residential changes as well (Perner, 2002).

Outreach. The subject community college provides outreach services to the feeder secondary school. These outreach services take the form of counseling services, presentations, participation in transition fairs, college visits, priority registration, precollege classes and ongoing communication through both mail and email systems. Professionals were aware of the services offered and one student acknowledged extensive mail and email outreach. Outreach technician Barbara Wood unpacked the services provided by the community college:

We provide a range of services for high schools wanting to help their students transition to our college. We have people from counseling who will come out and make presentations to the seniors, we are available for transition fairs that districts might hold, and of course, I am presenting in individual classrooms. There is also someone from counseling here in the library at lunch on Thursdays to answer questions and enroll students.

In addition to these efforts, English teacher Doris Hall related, "For any of our students they do priority registration, they take them and show them around the college campus. They actually do, like, precollege classes over the summer." Outreach was mentioned by one of the nine students. Kim shared, "The college reached out to me all summer long. They sent me emails, they sent me a few letters. I can't really remember what they were for, but the main thing was the emails". 
It is clear that the outreach technician saw the many ways that the community college attempted to connect with students who might consider attending their institution, and there was an awareness of outreach by English teacher Doris Hall, as well as one student. Whether only one teacher and one student acknowledging outreach was indicative of a lack of general awareness was uncertain; still, the presence of outreach mechanisms has the potential to facilitate student awareness of the community college, thereby promoting transition to the institution.

Reliance on Grant Funding. Though the community college offers mechanisms to promote transition to the institution, the outreach technician and on-site counseling services were reliant on grant funding rather than the regular budget for their existence. Outreach Technician Barbara Wood related, "No, this is the first year. It's funded by a grant - this is brand-new...so with that, they decided to hire certain people to help with these transitions. The grant freed up other funds which supports the counselor on Thursdays as well".

Utilizing grant funds to offer transition services creates a mechanism whose continuing existence is subject to the vagaries of uncertain financial resources; programs are introduced as funding is obtained and can be terminated if funding expires. Such an erratic approach to transition services inhibits the development of a robust, ongoing transition program.

Lack of Understanding. Findings indicated that there was a lack of understanding of the needs of students identified with autism within the community college culture. This lack of understanding was expressed by one student when describing their interactions with DSPS staff; it was apparent in the manner in which 
DSPS administrators discussed students identified with autism, it was present in the stigmatizing approach taken by the school regarding equity, and it was evident when two of the nine students related their frustrations with the DSPS facilities provided for them. Briana explained how students identified with autism may not be receiving the support they need from DSPS:

I mean, don't get me wrong, like, DSPS has been really helpful and stuff, but at the same time with people like me where I have an invisible disability...you know, you have to go into the office into the special back room and everyone who works there talks down to you and treats you like you're dumb or lazy... Or when they see people that don't look like they have a disability they're like, "Oh, why are you a part of this?" So, in that sense it's like, because it's like this little niche thing - it's like, almost instead of integrating us into the school more it makes us stand out, makes us different...

This othering was evident in how the college views the DSPS program. DSPS Coordinator Linda Adams viewed DSPS in terms of equity:

We certainly are looking at data in terms of equity and our equity plan, and so I think, that---things are looking pretty good in terms of looking at that AA degree attainment. I think one of the things that we do know is that there is an equity gap in terms of students with disabilities transferring to four-year institutions... The college is engaged in all kinds of work to try and improve outcomes for those students where there are equity gaps, and, you know, in many cases, of course, that is going to be true for our students with disabilities. 
The participating community college published a Student Equity Executive Summary 2017-19 (Appendix K, redacted for anonymity), in which they identified student groups where equity needed addressing. These groups included students who identified as: African American, Asian, Filipino, Pacific Islander, Latino/a, White, Native American/Alaskan, Male, Foster Youth, Veterans, Low-Income; students who marked "Other/Decline to state", or "More than one race" on their CC Application and students who had a documented disability. Briana experienced a confusing stigmatization in reference to the compilation of these groups:

That's like... for people who may be financially, or... like intellectually, like, lacking? In a way? Anyway, and they identified...I'm honestly not a hundred percent sure how they identified that I was in that group... But it was, like, when I went to registration, I automatically got put in with all the disabled students and the financially...poorer people...

The lack of understanding was further evidenced in the lack of clarity over what a student with autism needs and how these needs can be addressed. Dr. Robert Miller stated in reference to students identified with autism, "Sometimes students have not been assessed and so we want to assess them for an LD right then and there." DSPS Coordinator Linda Adams expressed reticence in providing the services needed for students identified with autism:

Ah, we did have a situation come up even recently, ah, it was, you know, an individual with an autism spectrum disorder, who wanted to have, again, an attendant in class, and the purpose of which was to provide instructional support. That was a very difficult situation for us because that is just...there is...we, we... 
there is a balance we need to have here at looking at what the student needs and, and then at what, what a student ideally would be able to do, do as an independent college student, and, and that can be difficult.

The environment specifically provided for students receiving services through the DSPS office may not meet the needs of students identified with autism. As expressed by Briana:

I also been given - they have a room where they can do testing but honestly that's, that's like - it's a good thought they have, but they don't plan it out very well, because you have to schedule an appointment like super in advance and often times there's like 10-15 kids in one room, so it's like you're really not getting privacy...

This view was echoed by Martin:

What else could it (DSPS) do? I think that the disability center needs to be given its own building, if not at least a bigger space. I feel like the DSPS office is too crowded and loud. It's supposed to be there to assist me, but I often struggle with just walking through the door because of the environment. I think it's important for students with disabilities to have a quiet, low stress environment and I don't think West College is providing that right now.

These findings indicated an educational environment that had no clear approach to providing services for students identified with autism. Students relayed experiences where personnel were othering and questioning rather than welcoming and accepting; they experienced the stigma of being grouped with the intellectually and financially "lacking"; they were provided with testing environments that were inconsistent with their 
sensory variances. These actions and assumptions created a discriminating environment that can lead to desistence.

Rather than addressing this discrimination and providing an environment that fostered success, administration wondered aloud why the college has an equity shortfall relative to "students with disabilities". This lack of understanding by administration goes beyond Linda Adams and her focus on tackling equity issues and removing academic supports over addressing the variances present through appropriate mechanisms; Dr. Miller conflated autism with LD, demonstrating a basic lack of understanding even within the medical model. In viewing disability as just one of many equity shortfalls; the community college district has created a situation where students become confused and stigmatized and perceive themselves as being othered.

Lack of understanding by the community college as to the specific needs of students identified with autism resulted in a generalized approach to services for these students. These generalized services can be counterproductive and stigmatizing, thus leading to frustration and potentially, desistance.

Faculty Shortfalls. Findings indicated that though DSPS coordinators generally viewed the community college faculty as supportive of the needs of students identified with autism, there existed among the faculty those whose views are incompatible with the needs of these students. Contrary to the views of the DSPS administration, findings emerged that highlighted faculty who espoused rigorous vetting and segregation for students with autism, and other faculty who believed that developing social and communication skills for these students was the responsibility of the high school rather than the college. Still other faculty believed that it was their responsibility to work 
effectively with students identified with autism but reported that they have received no training in how to do so.

DSPS Coordinator Linda Adams presented a picture of easy cooperation with the college faculty when she stated, "We do not run into very many difficulties with faculty. I think, comparatively speaking, we have a pretty good situation in terms of faculty understanding that accommodations need to be provided to students". Dr. Robert Miller agreed with Linda Adams and further presented information on new faculty training in stating, "Every new professor that comes in, we will have some time to talk with them to ensure that they are familiar with the services available and the expectations of the college relative to students seeking assistance". From the perspective of these two administrators, the community college is an institution where faculty are in general agreement with the aims of the DSPS office and the needs of students. However, of the three community college faculty members interviewed, two expressed views inconsistent with the needs of students identified with autism. Christine Wilson believed that students identified with autism should be thoroughly vetted prior to admission to community college:

I believe that part of the transition process should be a thorough vetting of these students to ensure that the autistic student that chooses college is prepared and suitably tempered for college.

Ms. Wilson's notion that students identified with autism need to be assessed to ensure that they are "suitably tempered" smacks of medical model ableism. Access to community college should not be based on a discriminatory, conformist notion of 
'temperament", but rather what the college can provide to the student and through the student, to the community at large.

Beyond vetting and assessment for temperament, Ms. Wilson further held that students identified with autism should be in segregated settings:

Some might disagree, but I believe the best way to address the needs of autistic students is to as much as possible provide them with specialized settings. This helps the autistic student because they are able to get the support they need, and it helps the regular college students because we the don't have to spend extra time addressing the needs of the autistic student in the regular college classroom.

Fred Richardson viewed the sociocommunicative variances of students identified with autism to be a secondary school responsibility:

I think they need more help, and I think that help needs to happen at the high school level. If a student has problems communicating, they need to have extra help learning how to communicate while still in high school. If they have problems socializing with others, they need to learn how to socialize better while still in high school. Once they get to college it's really too late to learn these things.

Community college teacher Terry Edwards expressed concern regarding applicable training:

I don't know what accommodations are made for the full-time faculty to be trained regarding the needs of the students, but I do know that part-time faculty receives no training whatsoever. I am part-time faculty, so I should know. 
The views expressed by Linda Adams were generalized in nature; Dr. Miller's statements were somewhat more specific. Whether their statements were intended as public relations optimism, or whether they were unaware that some faculty held views inconsistent with theirs is uncertain. What is certain is that the views held by Christine Wilson and Fred Richardson are contrary to the success of transitioning students identified with autism. A student identified with autism who might find themselves enrolled in one of their classes could well experience not just a lack of positive affirmation, but potentially outright hostility towards their presence and/or particular variances. Such hostility could contribute to a lack of persistence at the community college. For teachers such as Terry Edwards, a willingness to support and affirm students was hobbled by a lack of training, a contention in opposition to the statement made by Dr. Miller. Together, there existed a considerable disconnect between the views of administration and those of faculty relative to support for students with autism; this disconnect likely resulted in frustration and eroded support for transitioning students.

\section{Student Struggles}

As students transition from secondary school to community college, they often struggle to detach from high school, adjust to a new norm, integrate into the community college environment, and develop supportive and nurturing mechanisms that provide validation for their new experience. As they approach graduation, they may experience anxiety over their unknown future and concern over their ability to be self-reliant. Throughout the transition, they may express concern over their ability to integrate socially and communicate successfully within the environment that loss of high school peers and introduction of community college creates. Further, they often wrestle with the 
changing environment and its impact on their ability to navigate the sensory challenges it presents. Finally, they may acknowledge the institutional support and guidance received at the community college and the impact that support had on mitigating their struggles.

Anxiety. Seven of the nine students indicated some level of anxiety surrounding departure from high school. This anxiety presented itself as a generalized concern for the unknown, apprehension over the need to be self-reliant and advocate for oneself and a loss of routine/structure. These anxieties have the potential to derail student success and persistence by promoting doubt and isolation.

“A Total Unknown”. Five of the nine students specifically addressed apprehension over the unknown during the interviews. For example, in response to being asked about the experience of finishing and leaving high school, George stated, "Well, I'm kinda nervous really yeah, I've never really been in a situation like this. I don't know what will happen next." Briana said, "I’ve always known what was going to happen next. Now I don't know.” Martin responded, “I like to know where I'm headed. This feels like a total unknown".

Anxiety over the unknown was equally a factor once transition to community college had taken place. In response to a question about places where the student feels less comfortable, Kim stated, "I'd say probably the buildings I've never been into. I don't know those buildings so I'm probably uncomfortable there." Chris responded, "Any of the other buildings" made him uncomfortable "...because I do not know much about the other buildings." 


\section{"I really don't feel that comfortable at school" - Concern over self-}

advocacy/self-reliance. Four of the nine students expressed concern over their ability to advocate on their own behalf and be self-reliant. Concern about self-reliance was addressed by Briana. When asked where she feels most comfortable on campus, she stated, "I feel the most comfortable in the library because it's quiet...the security office is right in the library, so it makes me feel more comfortable as well.” When asked where she felt less comfortable, she stated:

I feel less comfortable when I am having to walk across campus, like in the quad area or when I am in the student parking lots. I always feel more vulnerable and unsafe in these areas; I sometimes get the feeling that I am being silently judged by my peers. Also, I feel as though our school parking lot is unsafe for both drivers and pedestrians, plus the loud noises of cars often startle me.

When asked if she stays after school, she responded, "I always leave as soon as possible. I don't stay because I really don't feel that comfortable at school and I don't have any friends; I struggle to make friends, so there isn't a lot of motivation for me to stay." Steven perceived self-advocacy as a personal challenge to be formally addressed. When asked about his class schedule, he stated, "I take self-advocacy (class) on Monday and Wednesday." Kim expressed that she had had success in advocating for herself at community college. She stated, "Yeah, I notified both of them (her teachers) that I was part of the DSPS program...”

"It's Going to be Weird" - Loss of routine/structure. Two of the nine students referenced loss of routine or structure during the interviews. In response to being asked how he felt about graduation, Chris stated: 
So, it's like, I don't have to worry about all the stress from high school, about like all the final things I have to do or the big projects, but I will miss, I guess, the structure of it...Like you come here at seven in the morning and you leave whenever you finish your classes.

In response to looking back at graduation, Chris commented, "For me the experience of finishing and leaving high school was such a strange thought for me...that being changed felt weird to me because of how I can choose what to do next". For Amy, loss of routine meant adjusting to a different school setting. She stated, "The school is big and a little scary, but I am getting used to it. It's just new with a lot of different people. A lot of people I don't know."

The anxiety these students reported, whether expressed as a concern over the unknown, one's ability to be self-reliant, or loss of routine, share as a common thread the change in environment these students anticipated after transition. A preference for routine is a documented characteristic of individuals identified with autism (Lee et al., 2014); departure from secondary school is a sweeping loss of routine whereby friends, setting, teachers and schedule abruptly terminate. The anxiety felt by these students was a natural consequence of this disruption.

Each student experienced their own degree of anxiety relative to this change based upon their mechanisms for coping and their emotional variances. Some students acknowledged the anxiety but were not focused on it; others experienced an ongoing anxiety that must be continually mitigated through choice of setting and minimizing time on the college campus. 
Acknowledging this anxiety is important; by doing so, mechanisms can be devised to ensure that the transition experience addresses these concerns in a manner that fosters social integration and persistence within the community college setting.

Sociocommunicative Challenges. Five of the nine students interviewed related challenges they faced in communicating effectively in social settings. These sociocommunicative variances have the potential to affect student success within the community college by resulting in misconstrued social interactions, leading to the student's alienation and sense of isolation.

When asked about her views relative to her own communication skills, Briana stated:

I have a hard time communicating with my peers and making friends. I mean, I know I don't pick up on social cues, but I also know that my brain just works a lot differently than other people; so sometimes I am having conversations with people and they don't understand what I'm talking about and they think I'm weird...I do feel to a certain extent like weird and like alienated from my peers... When asked later in the interview how her experience in community college had been so far, Briana responded:

So far, I have been okay with community college... I do wish that for people like me, they could make the whole registration process easier. It isn't always easy for me to go in and meet with adults, so if I had the opportunity to communicate more via phone or email or a message portal, it might make it easier for me to say what I need and ask for help. 
George held out hope for improvement in his social interactions. He stated, "I mean, yeah... Getting along with others tends to get better as you get older. Like my problems here isn't as bad as the problems I had when I was in elementary school...". Fred indicated that he addressed his communication variances by minimizing social interaction with other students. He stated, "I am mostly focusing on my studies. I haven't really had time to socialize with other students, but I do talk to the teachers as often as I can so that's a good thing." When asked about places that make him feel less comfortable, Martin related, "For the start, when I first got to my first day at class, they introduced themselves, for about an hour. That was difficult." Martin further alluded to his social discomfort when he stated:

The most comfortable place would be the computer lab...there're less people, so it will be less crowded...the students there, of course they come in at any time so like I'll go in in the mornings at $9 \mathrm{AM}$ and there are like no students there.

Chris shared his concern over the challenge of new environments:

...there's going to be people from my high school there at West College, but there'll probably be people there that I don't know and haven't talked to before. I am getting nervous about starting like I was when I started high school because it was a new place with new people.

After a few weeks at community college, Chris related, "I leave right away because I do not know many people at the college." Briana chose to select settings where social interaction was restricted, stating, "I like to hang out on a comfy chair in the library...it's quiet, everyone is focusing on their schoolwork or studying, and no one bothers you." 
For those students who reported challenges relative to social interactions and effective communication, the common feature was avoidance; students pursued the development of mechanisms that allowed them to circumvent social exchanges. Whether it was communicating by email, focusing on studies to the exclusion of social engagement, or finding a quiet place to study in the library, these students sought immediate remedies to evade sociocommunicative interactions.

While these avoidance mechanisms temporarily managed the social challenges, they did not promote assimilation into the community college for these students, neither did they facilitate the incorporation of improved social skills.

Departure from secondary school can result in the fading of one's longestablished social circle; the avoidance reported by these students was the result of the introduction of a new, imposed social environment. These students utilized this mechanism to side-step social discourse; whether this was a temporary approach while they acclimated to the new environment, or whether this became the chronic mechanism was uncertain, but the ongoing use of avoidance as an approach will likely have led to increased social isolation and potentially, desistance from the community college setting.

Sensory Challenges. Two of the nine students commented on sensory challenges relative to their transition experience. The impact of sensory variances for students identified with autism has been well documented (Prince-Hughes, 2002; Adreon \& Durocher, 2007; Gillot \& Standen, 2007). The degree to which these sensory inputs impact an individual student varies but can be significant; ongoing sensory challenges can result in the community college environment being perceived as inhospitable and 
lead to avoidance and ultimately desistance. Briana, in responding to a question about what DSPS could do better, commented:

I feel like the DSPS office is too crowded and loud. It's supposed to be there to assist me, but I often struggle with just walking through the door because of the environment... It's important for students with disabilities to have a quiet, lowstress environment and I don't think West College is providing that right now. Martin related his sensory challenges when he stated:

The (florescent) lights in the classrooms bother me. They hurt my eyes and they hum a lot...the cafeteria can sometimes be uncomfortable because of all the people and there are a lot of options for food that I don't like.

For students with sensory variances, the challenge of enduring a crowded room with bright, humming lights can be unnerving; these environmental offenses can lead to the student avoiding such settings. If the offending setting is the DSPS office, counseling, admissions or some other critical location, they can create significant roadblocks to success and potentially result in desistance.

Support and Guidance. Six of the nine students referenced the importance of institutional support and guidance through the transition process. Rendón (1994) theorized that validation can promote social acclimation. In the case of these students, they reported an upbeat attitude towards teachers and the DSPS office. It is likely that such comments reflected a positive outlook that will promote persistence.

“I love my teachers". Five of the nine students specifically identified teachers as a source of support and guidance. Fred expressed the value he placed on the community college teachers when he stated, "This is a pretty big step (transitioning to community 
college) - I'm not gonna lie, but the teachers here, they are very nice and support me. They are here to help me.” He further stated:

I get a little nervous every once in a while, 'cause some of the stuff is super complicated and I' $m$ unable to do them, but the teachers are easy to understand, and they help me with it when I need it and explain it pretty well and that's how I'm able to stay on topic. They're all very understanding.

Briana expressed her enthusiasm for the community college teachers by stating, "I've really been able to communicate with my professors and explain my... situation with what accommodations I need, and everyone's been very understanding and very kind about it". In reference to a particular teacher, she reiterated her views by stating, "I also like it because the professor is very understanding and kind" and later returned to the topic by adding, "...to be quite honest I expected the professors at the community college to not be very good, but I have been so happy with all my classes." Martin related:

Yeah, from my experience so far, I think the teachers are more productive on trying to make me understand how to do problems or how the concepts work than in the high school, because they can as take as much time as they have just to teach you, even at the end of class. Yeah, the teachers are supportive.

Kim included the teachers within the greater college community when she stated:

From what I can tell, everybody is so nice over there and the teachers, I love my teachers and I make, like, sometimes I make funny comments, or I make a joke and my teachers are just like really loving; they accept my jokes. Also, my English teacher did say if there is an essay that you turned in late and you turned everything else in on time, at the end of the semester I will grade that one essay. 
Chris expressed faith in his professors when he stated, "I believe they are supportive and are willing to always help."

"The people there are very nice". Five of the nine students indicated that they had received support and guidance from the Disability Services (DSPS) office. For Amy, support began early in the process. She stated, "Well, first the community college accepted me, which was great. The office (DSPS) helped me register and get classes." She further related that identifying accommodations was part of the support she received from DSPS when she stated:

The counselor at the disability office sat down with me and helped me figure out what help I needed my classes, like extra time on tests and things, and then got me a paper I took to the teachers, so they knew I needed extra time and other things. This was echoed by Fred who noted, "They (DSPS) helped choose and arrange my classes. They were very helpful." Chris indicated his trust in the support provided by DSPS when he stated, "I feel comfortable in places like DSPS because I know they are there to help me out."

Four of the students specifically identified support mechanisms provided by DSPS. Briana addressed a range of services provided by DSPS, stating "The DSPS office has constantly reached out. Sending emails, providing planners, tutoring, academic counseling." Fred shared:

There are some papers that I need to give the teachers from the DSPS office to make sure that I get the accommodations that I need, like the notetaker. I have a notetaker in my English class - she helps me out -and as I mentioned before, I'm able to go there when I need help with my homework or extended time on tests. 
Amy related, "I'm going to be part of the DSPS - they are going to help me and give me tutoring with that" and Kim provided specificity to the accommodations:

Yes, so my accommodations are, I get one and a half times as much time on tests, and I'm also allowed to take tests in the DSPS office in the testing room. But just like any other student, I have to notify my teachers; I have to notify the DSPS center a week in advance minimum.

Fred focused specifically on homework assistance in stating:

I, I'm keeping up with my assignments. I'm currently, in the DSPS office. They, and the people there are nice as well, they help me out with homework. I actually have a homework hall there in a little while. They are also helping me catch up with all my homework - that's good.

Kim was enthusiastic about an academic coaching program offered from DSPS. She detailed:

Yeah, actually I'm a part of their academic coaching program. Basically, what they do is they check in with you once, sometimes twice, a week for an hour and then they ask like how are you doing, do you have any upcoming assignments, do you have any homework that you haven't finished; and they keep like a tab I guess on grades and everything, and then on the occasion they teach us about strategizing and taking notes and managing your time in writing everything down in your planner. It's a lot of fun actually; it's really cool and I've found I get homework done and studying done there easy.

Kim went on to relate that she saw the DSPS as a nurturing environment. She stated: 
Oh yeah. It's (the DSPS office) actually right next to the D building where I have math, so I can literally just go downstairs, my class is on the second floor, go downstairs, walk across, walk right in and done. I've been in there several times already, and it's always a positive when I go there.

The findings indicated that students generally enjoyed their teachers and that the teachers were accepting and accommodating of student needs. These affirming statements were reflective of positive attitudes towards their teachers. As teachers can have a significant impact on the overall classroom climate, students who have positive views of their teachers may be more likely to persist at community college.

Students reported generally positive attitudes towards the DSPS office, expressing approval of both their outreach mechanisms and the accommodations provided. Such approval is important because it fosters continued use of the services and accommodations, which likely promote continued success at community college resulting in ongoing postsecondary success.

Together these findings illustrated that for these students, the community college was a guiding and supportive environment. Such an outlook tends to promote academic success and persistence.

\section{The Essential Nature of Personal Support From a "Familiar"}

A critical finding of this study was that support of a "familiar" was an essential component of transition for students identified with autism. This "familiar" can take many forms; for some, parents serve in this capacity, encouraging or insisting that the children go on to community college, meeting with secondary school staff to facilitate transition, and otherwise supervising the entire transition process. Once the student 
begins attendance as community college, the parent may continue their support by providing transportation, tutoring, or financial aid. For others, support may have come from a member of the extended family, whether in the form of an aunt who served as a role model, an older cousin who was currently attending college, or even a new family pet who provided emotional support. Others saw value in the support of peers, whether they were neighborhood friends, long-distance associates, or simply the idea that past friends were attending the community college. For still others, an assigned mentor may have been the support they needed. Regardless of the nature of the "familiar", their presence was a consistent and essential component of transition for students identified with autism.

"they basically forced me to go" - Parents. The essential role of parents was recognized by study participants across the spectrum. Six of the nine participating students expressed that their parents played a role in supporting their decision to attend community college. For some, the parental role began with transition planning. In reference to the question of post-graduation plans, Fred stated, "Deciding to go to West College was more of an at-home thing with my mother and father". Others expressed a more hands-on approach to support and guidance, as Martin related:

They (parents) were important in this decision because first I was going to go to another college, but my dad stepped in and said it was way too far and told me that I would take two bus rides and it would've taken hours to get there. They were very supportive of going to college in general, very supportive. My dad never got to go to college; he's only got a diploma from high school, which he said it encouraged me to go. Yes... they basically forced me to go. 
Kim related a similar parental directive:

In the beginning of the year they pretty much told me straight up, "you're going to community college". I was pretty flustered by that, because I've been wanting to go to Chapman University ever since I went to Camp Chapman in like first grade and I really just...I wanted that. I wasn't going to be able to apply and transfer over to Chapman, but it took me a while and I finally accepted it.

The expectation of college was echoed by Briana who stated, "Attending college was just assumed. Pretty much everybody in my family has a college degree, so the idea of me not going was not even discussed." Amy related a less forceful approach to support and guidance, "Yeah, they encouraged me, they encouraged me. They want what they - what I think is best for me. They support the decisions that I make; they help in what I want to do..." For Chris, his parents expressed a clear, practical reason for supportive community college:

They had a big role in this decision (to go to community college) because like I said, I didn't know what I wanted to do. My mom and dad, my dad's a realtor and my mom helps my sister with staging and helps with a little business. My dad used to work with it, and they want me to kind of run it later, so they want me to learn more about business. Yeah, they are one of the biggest things that has encouraged me to go to community college...

The concerns of parents in the transition of their child from secondary school to a postsecondary setting were illustrated by Mary Cooper:

Well, at the beginning of his senior year I knew we needed to start working on it. I had spoken to one of the vice principals who deals predominantly with that and 
she started the ball rolling on that and gave us some options, and then it wasn't until the spring, but there was a transition presentation that we attended. It's hard when you have been so much a part of their school lives and then that is probably going to change when they graduate - I just needed to be sure that everything was done and set so that they could be successful at the community college.

The secondary school staff viewed the support and guidance of parents in the transition of their students to be significant. As secondary school counselor Stephanie Taylor related: I don't know - the parents are typically - that $30 \%$ that actually do go on, that's because those parents went over to the college and did it. Like all the meetings I've had with my parents they've come in to request that IEP, to request that psych report, to get that Woodcock-Johnson, and then they said okay now who's the contact over DSPS, who do I need to call, they're in here asking all those pertinent questions, getting those documents, and they're making these appointments and they're taking those next steps...

Case manager Joe Rodriquez held a similar, albeit grittier view:

It's always the parents - especially with special ed. Unfortunately, a lot of our kids are unmotivated. So, the driving force - $80 \%$ of it - is probably parents; either standards and expectations or it's just a matter of their environment. If the kids can stay home and play Xbox all day they're going to stay home and play Xbox, but if mom and dad say, "Hey, go to this JC or get out of my house because you're an adult and you're not going to school, take a pick", they usually go to the JC. 
Case Manager Frances Bennett saw transition as a time of increased parental involvement:

I think that the importance of parent involvement is grossly underestimated on this (transition); parents as a culture think that when their child graduates from high school you have a big old blowout party and your time as a parent or your heavy-duty involvement as a parent is over (agitated). With students with autism it's exactly the opposite; they're going to need twice the amount of support, twice the amount of involvement, twice the amount of follow through in order to make sure that they get things done.

Parental support and guidance can take a variety of forms once community college attendance begins. Bernadette Foster focused on the practical issues of transportation and academic support:

I think there was a setting where her father was getting ready to retire and he would be able to transport her because she didn't have a car and didn't have a driver's license. She was going need transportation, so it was decided that she attend a community college near her father's home...her stepmother is very, very good with math and her father is strong in academics so she gets a lot of support from them. I think that their tutoring of her has helped.

Claire Williams echoed the same concern:

She came to live with us while she went to college because the amount of financial or other help she would've received at the other house would've been negligible, whereas with my husband and I both being high school teachers, we 
were more on top of making sure that things got done when they needed to get done, and not allowing deadlines to slide in perpetuity.

Students spoke little of the guidance provided by parents once they began community college and focused instead on the support received in the form of transportation. Martin stated, "On a typical day I get school in the morning, my dad drops me off basically at the school" and Kim related, "There is a time when I am on campus after school while she (Mom) is on her way to come get me..."

"I spent a lot of time with my aunt" - Extended Family. Four of the nine participating students spoke of the significance of members of their extended families in either helping them decide to go to community college or providing support once they were in attendance. For Amy it was her aunt:

It was - well - I spent a lot of time with my aunt. She's like, one of my best friends and so it just kind of made me feel like I can be great like her because she's a nurse... she said that she went to community college first and then she went on to like a four-year college... she said that community college was like the best for doing that.

Chris chose community college because of a relative who was currently in attendance, “Family's another big impact, other than my parents, because my older cousin goes there at the moment- I think he's - I want to say he's on his first or second year.” Martin was influenced by a sibling:

One thing is, is that I didn't really come up with this, all these plans, I didn't really help myself with this college, these college plans. The real big help with 
me was not my parents, it was my brother. He has taken classes in the past and is getting ready to attend a four-year college. He's basically on the same path...

In the case of Kim, extended family included the support of a recently adopted pet:

One thing that totally changed is that I now have a dog..... my mom feels like it seemed like the right time for me to have her. She's teaching me a lot about responsibility and how to not only take care of her but myself.

“One of my friends named Luis used to come here" - Peers. Three of the nine participating students expressed that they had received guidance and support from peers. Martin stated, "One of my friends named Luis used to come here but not anymore. He's at North College and he told me to go there but I said it is way too far and I'm not sure if it has the right career for me." George related that a peer need not be physically proximate to provide the needed support and guidance:

I have one friend that I talk to a lot over the year. One thing he really told me about was your grade point average during high school doesn't really matter that much to get into a high-level college because you can just go to a community college first and then transfer to finish up your college. Yeah, my friend helped a lot with a lot of decision-making...he's in Connecticut.

Though not significantly influenced in choice of school by peers, Kim saw a benefit in the presence of peers at her chosen school:

Originally, I was going to try to get into Chapman or Boston or UCI despite them (friends) going to West College and then once I finally agreed to going, I thought “Hey, my friends are going - that's a bonus!” So, my friends were never really like the major reason I (decided to go there) - they were just one little part. 
Kim further related the support provided by peers once attendance at community college has begun:

They've been very, very supportive. They've given me a lot of advice about, like what to do when something goes wrong, or to try this. The main thing is just keep moving and just don't quit. If you fail, try it again.

“you know, probably a buddy system” - Mentors. Though none of the students expressed currently utilizing a mentor other than a family member or peer, the idea of an assigned mentor was expressed by several professionals interviewed. Case manager Lois Perry stated:

I don't know - you know, probably a buddy system, maybe not like what we have here - we have a peer system - the Link Crew, but probably for us case carriers to recommend - if they knew they were friends with someone else who was attending West College and has successfully navigated the system before, you know the first year possibly that - that could be something that would be helpful. This view was echoed by community college teacher Terry Edwards:

I don't how feasible this would be, but I think it would be wonderful if every student - not just every student with autism but every student - was provided with another student who has been here for at least a year and could serve as their guide and support... This might go far in alleviating the isolation that many incoming freshmen feel.

The study's findings indicated that for students identified with autism, the support of a "familiar" was a consistent and essential component of transition from secondary school to community college. For the plurality of students, this support was provided by 
parents. Parental support often began with establishing postsecondary expectations, followed with support through the application and enrollment process, and continued once community college attendance began with ongoing financial, transportation and academic support. Parents recognized their utility in this process, and secondary school professionals acknowledged the unique privilege parents hold in facilitating their child's transition: parents, above all others, have the tools and the ability to profoundly motivate their child through the transition process.

Findings indicated that extended family provided support and guidance through positive role models or providing emotional support; peers can also provide advice and support through transition, though not in as direct or profound a manner.

Unlike the parents, extended family, or peers, the use of mentors was an approach recommended by professionals but not identified as an alternative by students or parents. This was likely because the mentors recommended by the professionals are programmatic in nature, whereas the students tended to view support in personal terms. This does not mean that the mentor approach would be eschewed by students; rather, that it simply did not occur to the student as a possible support mechanism.

\section{Summary}

The study's findings revealed that the process of transition for students identified with autism is a complex, multilayered system viewed by the participants and stakeholders within the context of their individual perspectives and colored by their own standing within the process. The findings point to factors that can be leveraged to promote a successful transition for these students and hint at why so few who espouse an intent to pursue postsecondary education engage or persist in that endeavor. 
The culture and architecture of the secondary school creates an environment whereby transition is both promoted and inhibited; the high school celebrates postsecondary attainment in a manner that encourages all students to consider college to be within their grasp, yet they focus on four-year institutions, thereby othering the idea of community college. They showcase a robust programmatic architecture that promotes transition but rely on the individual efforts of staff to ensure the program's efficacy. They utilize a system of student classification that results in multiple pathways for students identified with autism to transition, thereby resulting in disparate transition services based on medical classifications rather than desire or potential to persist. Awareness of these aspects of the secondary school's culture and architecture can allow for the institution of mechanisms that reinforce promotional traits and diminish inhibiting factors, thereby strengthening the school's transition program for all.

Between the end of secondary school and the beginning of community college, a summer "gap" exists that presents a challenge; services offered by the secondary school abruptly end, and students find themselves in a social and academic void. This unstructured period is often occupied by students with activities unrelated to postsecondary education; the challenge inherent in this "gap" is that students may not regain academic focus, thereby negatively impacting transition.

As in the case of the secondary school, the culture and architecture of the community college can both promote and inhibit transition for students identified with autism. While the community college DSPS administrators expressed a desire to nurture students with autism, and a plurality of students expressed that they saw the college as a nurturing environment, these same administrators seemed uncertain as to the needs of 
these students or how to best address these needs. The geographic proximity of the community college to the secondary school made it a clear choice for some students while affording the college an opportunity for convenient outreach, yet the dependence on grant funding for such outreach makes its ongoing presence uncertain. Additionally, faculty reported that they are either unreceptive to, or untrained in, mechanisms and strategies to best serve these students.

Students viewed the process of transition through the anxieties they endured, the sociocommunicative and sensory challenges they faced, and the nurturing feelings they experienced. An understanding of these struggles and affirmations can foster the development of transition mechanisms that mitigate their struggles, enhance their positivity and ultimately contribute to student persistence.

Throughout the transition process, students benefitted from the presence of a "familiar"; someone whom they knew well and could provide the guidance and support necessary to assist in the transition. For many, this may be a parent who provides the academic, transportation, and financial support necessary to promote successful transition. For others, extended family have served as role models. For still others, peers provided guidance and support. Additionally, mentors were thought of by professionals as a programmatic "familiar" that could be of benefit during transition.

The findings from this study as highlighted above provide insights into the transition process for students identified with autism as they graduate from high school, experience the summer "gap", and begin their first semester in community college. These findings will be discussed in the following chapter. 


\section{Chapter 5: Discussion}

This study explored and investigated the experiences and perspectives of students identified with autism, their parents/caregivers, faculty/staff and other key parties as they transitioned from secondary school to community college. The review of the literature revealed that much is unknown about this transition. What research has been conducted was generally bifurcated into either what the secondary school could provide or what the postsecondary institution could provide; a unified approach had not previously been addressed. Given the dearth of research previously conducted, and the intent to develop a unified perspective from the several discrete participating groups (students, parents, teachers, administrators), it was determined that no one existing theoretical framework would suit the parameters of this study. For this reason, this study utilized constructivist Grounded Theory methodology. Utilizing Grounded Theory provided the needed flexibility to develop an awareness of the transition experience from the perspective of the participants. This flexibility and awareness further resulted in the emergence of thematic elements that were foundational to an understanding of the process of transition as delineated within the study. These foundational elements are (1) secondary school architecture and culture as it promotes or inhibits transition; (2) challenges inherent in the summer "gap" between secondary school and community college; (3) community college architecture and culture as it promotes or inhibits transition; (4) student struggles and affirmations within the transition process; and (5) the value of personal support through the process by a "familiar". These foundational elements, discussed within, provided a preliminary understanding of the mechanisms that impact transition, identify potential factors that can be leveraged to promote a successful transition, and provided an initial 
understanding of why so few who espouse an intent to pursue postsecondary education engage or persist in that endeavor. This chapter restates the study's research questions, provides a discussion of the findings and considers implications, limitations, and recommendations for future research.

\section{Review of Research Questions}

The research questions that informed this study were

1. What are the critical factors that influence college trajectory students identified with autism as they transition from a secondary school to a community college?

2. How do students with autism and other stakeholders experience this transition?

The results of the study provide introductory answers to these questions. These findings and concepts are discussed in the remainder of this chapter.

\section{Secondary School Architecture and Culture}

Several key findings were critical to understanding the foundational role that the secondary school culture and architecture play in promoting and/or inhibiting transition to community college for students identified with autism. These findings included afflictive messaging, shortfalls between the programmatic architecture for transition and the practical implementation of such architecture, and an emphasis on the medical model that classifies students into categories, thereby resulting in discrepant and sometimes inappropriate service variances between individuals.

Afflictive Messaging. It was found that the secondary school under study places a well-documented emphasis on celebrating postsecondary attainment. This focus on 
postsecondary attainment was confirmed by a secondary school administrator who posited that the school emphasis reflects the wishes of the highly educated parent population within the community. This celebration of postsecondary attainment has the effect of sending a message to the students that this is the expected path.

Overlaid on the postsecondary messaging is a long-standing message of focusing on four-year institutions. The public documents reviewed made no reference to community college whatsoever and instead focused on universities and other state and private colleges. The school news magazine article that listed the graduating seniors and the postsecondary institution they will attend was structured in a way to emphasize the universities and Ivy League schools and obscure the community colleges, even though a plurality of the students listed community college as their postsecondary choice.

This emphasis on four-year institutions was confirmed by staff, who related that the traditional view of community college within the secondary school is negative. To what extent these negative views are relayed to students in classrooms is unknown, but at least one student articulated that consciously or unconsciously, secondary school staff were encouraging students to select four-year institutions. Taken to the extreme, one teacher related the tale of two students who actively represented their acceptance to Ivy League universities though their academics would not support such a selection and it was too early in the year for anyone to have been selected.

These two students highlighted the risk associated with a robust emphasis by the secondary school on four-year institutions as the preferred choice of postsecondary destination; students may assume a façade of university acceptance and intent to enroll for the purpose of conforming to perceived standards. Though no research directly 
addresses this façade of university acceptance while in secondary school, Roschelle and Kaufman (2004) reported that in order to avoid stigma, homeless youth will engage in strategies such as "passing", whereby they assume the façade of having a residence. For students transitioning out of secondary school, such façades can result in students asserting false postsecondary intentions, thereby potentially inflating perceived enrollment numbers. The false claims of these students bring to mind the study conducted by Wagner et al. (2007), that highlighted the large percentages of students with stated intentions to pursue a postsecondary education. Whether some of these students inflated their intentions is unknown, but further study relative to goal inflation might shed light on this aspect of student reporting.

For these students, their potential inflating of postsecondary intentions was less important than the possibility that their "faux" acceptance might cause them to delay action needed to successfully enroll in realistic postsecondary options. This delay might have resulted in the loss of valuable transition services and impacted their successful incorporation into the community college environment, possibly leading to desistance.

The implication of the secondary school's messaging is that there is a preferred pathway for students to follow after graduation. For this secondary school Ivy League, university and four-year institutions are lauded; any other choice is viewed as settling for less. For students identified with autism who intend to pursue a postsecondary education, this creates an uncomfortable alternative. If they attend the proximate community college it allows them to live at home and address social acclimation without concurrent residential disruption but they must contend with disapproval by the secondary school culture; if they attend a four-year institution in keeping with school expectations, they 
enter an environment where they might need to endure multiple social challenges. This specific issue was not addressed in the research, but Holland (n.d.) addressed the challenge faced by less advantaged students in similar circumstances and reported that they either choose community college and develop mechanisms for avoiding the stigma or opt for a four-year institution and contend with the challenges of expense and inadequate preparation. Both options are poor and do little to meet the needs of students.

Understanding the factors that drive the secondary school in their messaging requires that one review some of the history surrounding postsecondary education. As related in Chapter 2, Humes (2006), wrote that postsecondary education was the domain of the elite prior to World War II. As a benefit of military service, President Roosevelt made a four-year postsecondary education within reach of the middle class with the Servicemen's Readjustment Act of 1944. As hundreds of thousands of newly discharged servicemen availed themselves of what was known colloquially as the GI Bill, the longterm result was a dramatic increase in living standards and a realization that a collegeeducated population was good for America (Humes, 2006). As the Pew Research Center (2014) report indicated, the benefits to college graduates remain strong and pervasive. Unfortunately, an outgrowth of this mindset has been a muddling of what college means; according to research by Holland (n.d.), the "'college-for-all' norm was interpreted as 'four-year college-for-all'", resulting in stigma being attached to attendance at community college.

This stigma needs to be addressed; direct attendance at a four-year institution should not be the only path endorsed by the secondary school. A more nuanced definition of college is called for. Practitioners and school officials should embrace not 
just university-bound students but all students regardless of their destination. To assist in this endeavor, secondary schools need to facilitate multiple pathways for transition, not just four-year pathways. How this is accomplished becomes the challenge: little research has been conducted addressing such an approach; research on facilitating transition relative to postsecondary goals often reinforces traditional attitudes by addressing strategies for increasing four-year enrollment (The College Board, 2008; Hill, 2008; Morgan, Sinatra, \& Eschenauer, n.d.).

The secondary school should assist students in determining their long-term goals and, having made those determinations, provide them with the transition assistance necessary to achieve those goals. For students identified with autism who wish to pursue a community college education, the school culture should value that choice and provide mechanisms that facilitate that transition. Holland (n.d.) recommended that teachers and counselors present the community college in a positive light while promoting stronger academic preparation. For students identified with autism, enhancing sociocommunicative preparation while maintaining academic preparation may provide an effective blueprint for success.

This does not mean that we need to value four-year institutions less; we need to value all postsecondary alternatives that facilitate student aspirations equally. This would be consistent with statements by the Secretary of Education advocating apprenticeships and other workplace learning experiences (Klein, 2017), as well as those by former Secretary of State Clinton who stated, "College is crucial, but a four-year degree should not be the only path to a good job" (Hillary Clinton Acceptance Speech, 2016). To do this, students, staff, and parents will need to be reminded that student goal achievement 
takes precedence over prestigious postsecondary enrollment. Documents meant to highlight school success need to be revised through the lens of successful transition, rather than university admittance. Regularly profiling former students who have chosen community college as well as other pathways and have succeeded would be one way of demonstrating to the school community the value of such choices.

Researchers would do well to explore how such a re-envisioning of a school could be achieved. While research has been conducted that aimed to identify changing school culture to promote college attendance (Rochford, O’Neill, Gelb, Ross, \& Stark Education Partnership, 2011), research addressing promoting options in lieu of four-year institutions has not; an understanding of the challenges faced, and successful strategies utilized, would be of enormous benefit to the secondary school leadership and classroom practitioners as they attempt to refashion the school to create a culture that telegraphs an inclusive, consistent and embracing message.

\section{Shortfalls between programmatic transition architecture and practical}

implementation. The secondary school under study embraces a robust programmatic architecture of transition. For students with IEPs, there is an integral ITP mandated by IDEA that begins prior to their $16^{\text {th }}$ birthday and is reviewed annually through $12^{\text {th }}$ grade. Presentations are offered by several community colleges in the fall for seniors, and the participating community college offers on-site assistance in counseling and enrollment during the last quarter of the year. There is also an outreach technician who is available for classroom presentations. Finally, community college classes are offered on the secondary school campus. Taken in total, the appearance is that of a school that offers a rigorous transition program. Closer inspection reveals a somewhat different reality. 
A critical finding relative to an understanding of the shortfalls present in the architecture is that it was heavily reliant on the efforts and initiative of individual teachers for effective implementation. For students with autism who have an IEP, the efficacy of the ITP was heavily reliant on how the individual case manager approached the development of that ITP. The structure of the documents, administrative oversight and a desire to avoid litigation can result in case managers preparing documents that are so formulaic that they become more of a bookkeeping document than a legitimate plan for transition; more a document written to avoid missteps than to provide next steps.

Szidon et al. (2015) addressed this issue tangentially when they recommended that specific postsecondary transition goals be identified through assessment and be linked to IEP goals in an authentic fashion. Implicit in these statements was the acknowledgement that the preeminent goal of an ITP is a useful, appropriate and effective transition. While the case managers within this study would likely agree in principle with this assertion, they found themselves pressured by other forces that may run counter to these goals. As Frances Bennett related, the case managers within the school under study want to ensure that they provide realistic, obtainable goals that are helpful to the students, but they feel constrained by district procedures, state and federal regulations, and general angst over potential litigation that might result from imprecise or improperly nuanced verbiage.

The effect of this emphasis on regulation and process in the development of the ITP was that the document became defensible against potential legal action by a parent but might not provide the authentic transition guidance and assistance needed by the student. As a result, the efficacy of the transition documents was placed at risk. 
While the fall assemblies for the visiting community colleges were clearly recognized by the secondary school as a programmatic mainstay of transition, the findings demonstrated that this mechanism was highly informal and resulted from the actions of an individual teacher. It is laudable that this teacher takes upon himself this additional responsibility, yet there was no mechanism in place to assure the continuation of this aspect of the architecture should this teacher retire, leave or otherwise be unable to continue facilitating this mechanism. Further, there was no research addressing this phenomenon or how a personal responsibility might be effectively transitioned to a programmatic mechanism.

Fall assemblies and classroom presentations were available for students, but there was no assurance that individual teachers or their classes of students attended or otherwise availed themselves of these offerings; findings indicated that teachers did exclude their classes from these assemblies because they valued their instructional time more than any potential benefit of the assemblies, and access to classrooms for presentations was dependent on the largesse of the individual instructor.

Protecting classroom instructional minutes is an admirable goal. What is sometimes referred to as "time on task" grew out of the work of Carroll (1963), in which he identified perseverance, or "the time the learner is willing to spend in learning" as one of five elements that contribute to effective instruction. However, as Stallings (1980) opined, "Clearly, student learning depends on how the available time is used, not just the amount of time" (p. 11).

No assessment can be made here as to the value of the instruction that might be missed by attending an assembly or allowing a guest speaker to present in one's 
classroom; what can be stated is that valuable components of the community college outreach and transition architecture were being neutered by the failure of individual teachers to allow their students access to the information.

Additionally, denying students access to community college outreach mechanisms may have the effect of dissuading students from pursuing such a postsecondary path; research by Ulug, Ozden \& Eryilmaz (2011) indicated that teachers' negative attitudes have a negative effect on both the performance and development of students. If a teacher by their actions devalues the importance of these assemblies and presentations, such negative attitudes can function as strong disincentives towards postsecondary enrollment, in the same way that community college faculty affirmation serves to enhance persistence as posited by Rendón (1994).

Doris Hall spoke extensively about her on-campus, zero-period community college class, yet no other secondary school study participant mentioned the existence of such a program. This researcher previously posited offering community college classes on the secondary school campus as a practical approach in response to the proposal of Zager and Alpern (2010), who recommended partnerships that allowed students with ASD to attend secondary classes on postsecondary campuses. A review of the literature did not reveal programs that provide community college courses on secondary school campuses specifically for students identified with autism, nor was any guidance provided as to programmatic mechanisms for implementing such a program. In the case of Ms. Hall's English class, whether the existence of her on-campus college offering went unmentioned through happenstance or whether there was some other factor in play is unknown. 
Previous research into institutional factors that impact students identified with autism has generally focused on more robust and targeted transition plans for students with IEPs (Roberts, 2010; Szidon, Ruppar \& Smith, 2015; Wei, Wagner, Hudson, Yu, \& Javitz, 2016) or providing specialized intervention through the counseling department (Dipeolu, Storlie \& Johnson, 2014). The research was devoid of findings that provided insight into how a secondary school can ensure that transition mechanisms are universally accessed and valued. The closest the research came to acknowledging the possibility of this shortfall was in reports that some students, including those with autism, may not receive adequate transition support due to a focus on those with more severe disabilities (Janiga \& Costenbader, 2002), or in the suggestion by Levinson and Ohler (1998) that students identified with autism have a condition that is so "mild" that they can succeed without intervention.

Though the secondary school provided services and mechanisms that can support transition, and individual students may have found themselves fortuitously benefiting from these services, a significant gap existed in the services offered; the results of this study indicated that the challenges these students faced in transition were predominantly socially driven.

There was an awareness among the secondary school professionals that students identified with autism may experience sociocommunicative variances, and students expressed difficulties with anxiety relative to the transition and being misunderstood in social settings, yet the study's findings did not indicate that there was any programmatic architecture in place to address these sociocommunicative variances. Research supported the need for programmatic incorporation of transition strategies, including self-advocacy 
and sociocommunicative skills (Wei, Wagner, Hudson, Yu, \& Javitz, 2016; Roberts, 2010).

Students identified with autism need sociocommunicative accommodations, opportunities to develop improved social skill sets, and a sense of empowerment relative to this variance. Dipeolu, Storlie and Johnson (2014) advised that school counselors could provide such training, but the time constraints and caseload typically assigned counselors, coupled with the potential "othering" of a specialized program, would make counseling a poor choice for overseeing such an endeavor.

In the absence of any architecture, providing these accommodations and opportunities for growth is dependent on the actions of individual secondary school professionals and their interactions with these students within their environments. This relegates the development of critical sociocommunicative skills to the arena of the haphazard.

The implications for students of these secondary school shortfalls is staggering; the school may offer these programs, but a student identified with autism might not be aware of these offerings or be afforded the opportunity to avail themselves of these mechanisms. Without access, these offerings, however robust, become ineffectual. As advocates for their children, parents need to actively engage with the school community to ensure that they are aware of the services offered and advocate on behalf of their children so that they can make use of these services. Research has shown that such parental engagement is more likely to lead to positive outcomes for students (Martinez, Conroy, \& Cerreto, 2012; Howard et al., 2016). 
Rectifying these institutional shortcomings falls squarely at the feet of the practitioners and the secondary school/district hierarchy. Case managers need to be provided with the training and support necessary to allow for the development of ITPs that are authentic tools for aiding in student transition while being fully compliant. Mechanisms for offering transition services should not be the domain of a single individual; redundancy should be built into the program so that a change in personnel does not result in a halt to services. Classroom teachers must be advised that attendance at transition assemblies and the allowance of classroom presentations relative to postsecondary goals are mandated activities that take precedence over classroom instruction. Better communication needs to be provided relative to the availability of onsite college offerings, though the research into dual-enrollment programs indicated that their impact on persistence is inconclusive (Swanson, 2008). Utilizing these approaches can help to mollify the impact of the shortfalls here presented.

Further research into the intricacies of the ITP and how the case manager can safely navigate the environment to provide a useful document while maintaining compliance and avoiding litigation would be of substantial use to the classroom practitioner. Further, mechanisms for integrating sociocommunicative training should be explored. Beyond this, researchers should investigate the mechanisms that result in some practitioners enthusiastically engaging with transition offerings and others demurring; understanding the factors in play could provide strategies for more successful engagement with all teachers, thereby mitigating the shortfall between the programmatic architecture for transition and the practical implementation of this architecture. 
The Medical Model and Service Variances. A critical finding emerged from an analysis of the data from this study; the medical model approach assigned students to pathways based on their diagnoses and resulted in their receiving discrepant and sometimes inappropriate accommodations and supports.

The findings revealed that "a student identified with autism" might have a medical diagnosis from an outside physician or a school psychologist that led to an IEP and being identified as a special education student, while another "student identified with autism" might have a medical diagnosis but - for any one of a number of reasons - have a 504 rather than an IEP. A third student may not have been diagnosed with autism but, as Frances Bennett states, "there's no doubt, the English teacher mentioned to me, there is no doubt that this kid has autism".

The deficit approach to the medical model requires a diagnosis of deficiency and a plan for treatment (Areheart, 2008). Within the secondary school under study, findings revealed that facilitating this treatment resulted in each student being directed into pathways based on their medically diagnosed or otherwise identified deficiencies. Students identified with autism might typically find themselves in any one of three generalized situations: they might have a medical diagnosis and an IEP and be receiving special education accommodations in their classes and perhaps taking self-contained special education classes; they might have a medical diagnosis and fall under a 504, whereby their classroom teachers have been informed of accommodations that are needed but with comparatively few supports or mechanisms to ensure those accommodation occur; or they might be functioning academically in a regular classroom, be undiagnosed, but be informally recognized by education professionals as "having autism". 
Within these three pathways there are students identified with autism who might wish to pursue a postsecondary education. The students have received differing levels of accommodations, depending on their diagnosis and academic performance. The accommodations provided by the secondary school often revolve around academic or sensory needs, such as additional time on tests, quieter settings, tutoring or help in comprehension. Little is offered to address the sociocommunicative variances. The student identified with autism who has an IEP and takes special education classes may find academic accommodations of significant benefit; the student with a 504 may receive sporadic benefit based on occasional accommodations provided and ensured by individual teachers; the student who is non-identified has made their way academically without these accommodations. The irony is that based on the individual actions of parents, medical professionals and to a lesser degree school staff, the same student identified with autism could potentially find themselves in any one of these pathways.

Transition as a component of the IEP is implemented at the secondary school under study in accordance with IDEA and other applicable law; these laws are driven by the medical deficit model. Accordingly, the secondary school attempts to rigorously follow the architecture set forth by the school district. This architecture ensures that actions taken follow all applicable regulations, that students will be categorized as appropriate, and that the accommodations will be meted out in accordance with past practices and the medical model.

The implications of these findings are profound; "similar" students identified with autism would be relegated to varying "lanes" of support and accommodation based on a medical diagnosis. These lanes would provide differing degrees of preparation for 
community college. Arguably none of these students would receive structured sociocommunicative support. This is unacceptable.

Students, parents, secondary school staff and district administration need to work together to develop mechanisms for supplanting these lanes of accommodation in favor of fully inclusive classroom settings. Research addressing fully-inclusive high schools is limited but indicated that such a program can be successful (Pierson \& Howell, 2013), is appropriate because it acts to respect all members of the school community (CaustinTheoharis \& Theoharis, 2009), and benefits all students regardless of their variances (Olson, Leko \& Roberts, 2016). Researchers should further explore what programs exist at the secondary level for fully including students and how those programs can be implemented in other settings. Recognizing that fully inclusive settings can create challenges for teachers (Symes \& Humphrey, 2012), researchers would also do well to explore what mechanisms exist or can be developed to mitigate these challenges.

Within these fully inclusive classrooms, rigorous, authentic supports and services targeted towards successful transition need to be provided on a consistent basis for all those who would benefit from such services. Providing the greatest support and accommodations only for those with an IEP enhances the likelihood that those students are receiving robust accommodations, but other students, by virtue of the vagaries of assigning pathways of support, who might also benefit from such assistance, are not offered it. Support needs to be provided not on the basis of assigned pathway, but rather on the basis of need and a willingness to embrace such support.

By utilizing such an approach, each student can be provided with the support and empowerment necessary to ensure that postsecondary transition, and specifically 
community college, is a viable option. Through these mechanisms, all students can be provided with the resources and tools necessary to aid in a successful transition.

Foundational Element Summary. The emerging image of the secondary school under study was an unsettling one; the school heavily promotes students choosing to attend a postsecondary institution but relegates community college to secondary status though the plurality of students reported choosing to attend such institutions. In appearance, there is a robust programmatic architecture to promote successful transition, but in practice the program was heavily reliant on the individual actions of teachers and could be easily circumvented or defeated by individual staff members. For students with an IEP, transition planning is a mandated component, but case managers reported the pressures under which ITPs are authored; for an unknown number, these pressures resulted in a document that was more a compliance device than a planning tool. Because of the convoluted mechanism for identifying and providing services to "students with autism", students with "comparable degrees of neurodiversity" may find themselves receiving wildly different accommodations and services. These aspects of the culture and architecture of the secondary school created an environment that both promoted and inhibited transition simultaneously; whether a student identified with autism perceived the secondary school as providing transitional supports or roadblocks depended heavily upon their diagnosis and resultant assigned pathway, their case manager's willingness to strongly advocate for them, the attitudes of their teachers regarding assemblies and guest speakers, and how the student perceived the messaging presented by the school relative to postsecondary attainment. 


\section{The Challenge of the Summer "Gap"}

Two critical findings emerged to form a foundational element for this study; the end of secondary school results in an abrupt halt to the routine and support experienced prior to graduation, and the "gap" that exists between the end of secondary school and the beginning of community college is often highly unstructured and can result in misdirected focus.

"graduation can be abrupt" - The End of Routine. Secondary school

professionals acknowledged the end of high school as an abrupt conclusion; the structure, the support mechanisms and the routine provided at the secondary school ends. Lee, et al. (2014), acknowledged the significance that routine has for students identified with autism. The end of secondary school is a termination of one's daily routine. While the neurotypical student may see the end of this routine as the onset of a degree of freedom, students identified with autism may view this loss of routine as a debilitating loss of control. Drawing from Schlossberg's Transition Theory, Lee et al. (2014) would advise that the student identified with autism address this loss by reinforcement of other factors of the $4 \mathrm{~S}$ model. In the case of graduation however, the student identified with autism experiences an immediate and permanent termination of both situation and institutional support; they will never return to the secondary school as a member of that social setting and they will never again have the support of the case manager, paraprofessionals, administration, other teachers, or the day-to-day support of students on campus. While family and friends outside of school continue as sources of support, the loss of situation and school support leaves the student with their personal makeup (self), and coping mechanisms (strategies) to compensate for this loss. Given that a preference for routine 
among students identified with autism has been previously established (Lee et al., 2014), as has a need to teach coping mechanisms (Roberts, 2010), reliance on these factors alone may be inadequate.

Research has indicated the importance of trust in helping individuals with autism adjust to new routines (Dalrymple, 1989). Given the loss of the school environment, parents need to leverage the trust they have with their children to both normalize this abrupt end of routine and facilitate the development of new routines that supplant those which have been lost. Research has found that parents can assume a coordinating or administrative role as well as provide emotional support through the transition process (Mitchell and Beresford, 2014), effectively serving as "mentors" for their children (Giarelli et al., 2013). This needs to begin well before onset of the summer "gap"; the most appropriate time for these actions will be determined by the needs of the student and the individual situation.

Within an inclusive classroom setting, secondary school teachers can discuss transition in a manner that normalizes the process while providing intentional, proactive affirmation, consistent with Validation Theory as presented by Rendón (1994). Case managers can incorporate more practical mechanisms for transition into their students' ITPs, including opportunities to develop comfort with loss of routine, approaches for establishing new routines and milestones to foster a graduated disengagement. Such mechanisms, though not specifically addressed, would be consistent with the recommendations of Chiang et al. (2012) and Szidon, Ruppar, and Smith (2015).

Secondary school and district administration should explore the development of mechanisms that could be offered to lessen the abruptness of the conclusion of high 
school while promoting movement towards postsecondary goals. For example, a student's post-secondary education goals could include summer transitional classes or trainings; independent living goals could include a periodic check-in with assigned mentors or support staff. In this way, the support mechanisms in place during secondary school do not simply disappear with the onset of summer vacation but are supplanted with other similar structures to provide needed support. This would be consistent with the recommendations of Kelley and Joseph (n. d.).

Additionally, research (Drouin, Reining, Flanagan, Carpenter, M., \& Toscos, 2018) indicates that social media (SM) "is viewed as a form of social support for the majority of college students" (p. 503). Limited research by Eckhardt (2017) indicated that individuals with ASD recognized social media as an avenue to communicate with other people and were open to interacting and getting to know others through various ways of communication. Given these findings, it is reasonable to suggest that researchers explore social media platforms to determine how they could be utilized to maintain contact between graduated students identified with autism and the secondary school in a manner that supports and eases the student's transition.

\section{"I've been doing a lot of sleeping" - The Unstructured Nature of the "Gap".}

For the student identified with autism who intends on attending community college, the abrupt conclusion of secondary school is immediately followed by a months-long period of waiting for classes to begin. The findings of this study indicated that this can be a time of unstructured activities or misdirected focus relative to community college attendance. Findings indicated that students pursued a range of activities to fill this time, anything from community service, to playing video games, to sleeping. 
The abrupt ending of secondary school, followed by months of unstructured activity creates an environment where students identified with autism, who relish routine, may lose focus and ultimately their way. As such, this "gap" represents a potentially hazardous period of uncertainty for students and can function as a powerful inhibiter to a successful transition.

Parents need to enhance engagement with their students during this summer "gap" to ensure that though the student may be taking a respite from the rigors of daily academics, they still maintain a focus on community college. Scant research into maintaining engagement for students identified with autism has been reported; strategies for low income youth (Terzian, Moore, Hamilton, Wallace Foundation, \& Child Trends, 2009; Perry, Khalilnaji-Otto, Brackenridge, \& Stanford University, 2018) tended to focus on elementary schools. The sole published study into maintaining postsecondary transition for low income students (Castleman \& Page, 2015) focused on institutions, advising that they use text messaging to remind students of required tasks and maintain regular contact through peer mentors, approaches that align with recommendations by Cochran (2009) and Eckhardt (2017). Additional research focusing specifically on students identified with autism and their experiences during the summer "gap" is called for.

Secondary school and community college policymakers would do well to develop mechanisms that could be implemented to facilitate a more seamless and robust transition. The traditional model of secondary school responsibility ending at graduation may not adequately meet the needs of all students. For case managers, providing validation by maintaining communication with former students could be all that is 
necessary to affirm the student and ensure that direction is not lost during the summer "gap"; emails could provide that connection with the past needed to let the student know that they are still valued and that someone believed in their ability to succeed.

For researchers, exploring and highlighting programs that promote a seamless approach that validates and thereby maintains engagement during the summer would be of benefit to practitioners. Additionally, researchers would do well to focus on developing an understanding of the mechanisms in play during this hiatus and how they ultimately impact actions to enroll or desist in community college.

Foundational Element Summary. The summer "gap" is the most perilous element of the transition experience. It is a time when the secondary school has lost contact with the students and community college has yet to actively engage with them. Further, this time is often filled with unstructured or misdirected activities. While summer "break" is a traditional component of the secondary school-postsecondary evolution, for students identified with autism the loss of routine and focus can result in a significant impediment to transition. Parents, policymakers and researchers each need to leverage their own strengths to ensure that students remain focused, transition is experienced in a less abrupt fashion, and the mechanisms in play within the summer "gap" are better understood.

\section{Community College Architecture and Culture}

Three critical findings emerged from the data analysis to form a foundational element relative to community college architecture and culture: the impact of a nurturing school environment, the lack of understanding within the DSPS environment relative to 
effective support for students identified with autism, and significant community college faculty shortfalls.

“I honestly feel very supported at school" - A Nurturing Environment.

Community College personnel often viewed themselves as supportive and nurturing of students identified with autism. These views were reflected in the comments of a plurality of the students interviewed. The students expressed their views in generally global terms, articulating their positive feelings about the teachers and the community college as a whole.

It is of significant value to note that all students who expressed positive feelings about the community college participated in all four interviews and were still in attendance at the conclusion of the study. Research has indicated that there is a positive correlation between student engagement and student success (Kuh, Kinzie, Cruce, Shoup, \& Gonyea, 2007; Upadyaya \& Salmela-Aro, 2013). These positive views about the community college and the students' continuing school attendance reflect the views of Rendón (1994), whose theory of validation supports affirmation leading to collegiate persistence.

There was little evidence within this study to support the idea that students identified with autism received more affirmation than any other student while in high school. Further, it was unlikely that students received significant community college affirmation during the summer "gap". While the exact causes of student desistance during the summer "gap" are uncertain, the value of affirmation in promoting persistence once in attendance at community college aligned with the work of Rendón (1994). 
Community college staff and administration can promote successful transition by integrating the validating and affirming mechanisms espoused by Rendón into their outreach and admissions programs. This should begin no later than the fall senior assemblies; students need to know in an explicit fashion that they are valued and wanted at the community college. Further, strategies need to be devised for the community college to ensure ongoing validation during the summer "gap". As mentioned previously in the case of the secondary school, social media use as recommended by Eckhardt (2017) and Drouin et al. (2018) could be of significant value in delivering affirmation through an approachable medium.

Researchers should consider leveraging the work of Rendón in developing targeted mechanisms for delivering affirmative messages to neurodiverse students within the community college setting, as well as exploring the summer "gap" from the perspective of the community college.

"everyone who works there talks down to you" - Lack of DSPS Understanding. A critical finding relative to community college culture and architecture was a lack of understanding within the DSPS structure of the needs of students identified with autism and how best to service those needs. Students reported being made to feel "othered" or otherwise deficient in their interactions with DSPS staff. Though there was evidence to support that the counselors within the DSPS setting are considered well-trained, scant evidence existed relative to the training of the paraprofessionals and office staff - those individuals most likely to come into regular contact with students.

DSPS administration inadvertently addressed this othering in discussing the school's equity plan; utilization of degree attainment as a benchmark and identification of 
those with a "documented disability" as needing equity assistance is little removed from identifying it as a medical model deficit issue. This lack of understanding further presented itself as a confusion over what autism is and a desire to withhold support in order to balance accommodations with an interest in having students function as "independent" college students. The attitudes of DSPS administration towards accommodation were clear: provide the minimum required, compromising as necessary, while moving students towards a neurotypical expectation of functioning "independently" without accommodation. This highlighted a mindset whereby there was only one approach and those who could not adopt that approach are problematic to the college. This was bewildering.

The planning of the DSPS facility highlighted a lack of understanding relative to the needs of students identified with autism. Students commented that the environment was crowded and loud, thereby challenging students' social and sensory variances, aspects of autism that have been well established (Prince-Hughes, 2002; Gillot \& Standen, 2007; Adreon \& Durocher, 2007); utilization of the testing room required significant advanced scheduling, often in opposition to research-based executive functioning variances (Prince-Hughes, 2004; Adreon \& Durocher, 2007).

The implications of these findings are clear; DSPS staff and administration should be provided with targeted training to better meet the needs of students identified with autism. Such training should include developing awareness of the prevalence of autism within the post-secondary setting, specifics related to individual challenges faced, and how best to accommodate and provide services for these individuals. Furthermore, mechanisms need to be in place to allow the DSPS department to regularly receive 
confidential feedback from their clientele relative to the services offered so that ongoing adjustments can be made as appropriate. Beyond this, changes need to be made to the facility itself. A larger, more inclusive site with a substantial area for quiet testing should be procured. Finally, the community college policymakers would do well to consider a renaming of the DSPS department to something less "othering". This would be best handled by engaging the affected students in the renaming process.

"We do not run into very many difficulties with faculty" - Faculty Shortfalls. While the views expressed by the DSPS coordinators portrayed a community college where faculty members generally function in concert with students and their accommodations, and every new faculty member is provided training on the services available, this study found that staff may hold views wholly inconsistent with the needs of students identified with autism.

Teachers play a critical role in the development of students identified with autism, especially in improving sociocommunicative skills (Helps, Newsom-Davis, \& Callias, 1999). Teachers' attitudes toward autism are critical for these students (Hadjikakou \& Mnasonos, 2012). Some research has been conducted into teacher attitudes surrounding disabilities, including learning disabilities. Studies have reported that faculty held negative, pessimistic attitudes (Minner \& Prater, 1984) and that students viewed faculty as insensitive to and unaware of their needs and reported feeling intimidated and rejected (Kurth \& Mellard, 2006; Wilson, Getzel, \& Brown, 2000). Other, more recent studies have reported that faculty members demonstrated positive attitudes toward students with disabilities and a willingness to provide accommodations (Austin and Peña, 2017; 
McKeon, Alpern, \& Zager, 2013; Gibbons, Cihak, Mynatt \& Wilhoit, 2015; Vogel, Leyser, Burgstahler, Sligar, \& Zecker, 2006), though these studies rely on self-reporting for surveys and questionnaires; as such, they may be more accurate in presenting how teachers wish to present themselves than whether they actually provide an affirming, inclusive classroom environment.

In this study two of the three postsecondary faculty members interviewed were hostile towards working with students identified with autism, either asserting that "autistic" students should be vetted prior to admission and taught in "specialized" settings or expecting their sociocommunicative variances to have been "cured" at the secondary school level. Though recommendations aligning with postsecondary vetting and specialized settings were not present in the literature, Elias, Muskett, \& White (2019) conducted focus groups with secondary and postsecondary stakeholders and suggested an “increased need to foster skill development to promote independence prior to postsecondary matriculation" (p. 263). The views articulated by faculty participants were in stark opposition to the open access mission of community colleges (USC, n.d.) and the "least restrictive environment" provision of IDEA, as well as the foundational views of the social model as set forth by UPIAS and others. These professors made it plain that they wanted to instruct neurotypical students in homogenous settings and they had neither the time nor inclination to adjust their instructional practices to allow for the inclusiveness from which a student identified with autism may benefit.

These two teachers are both full time employees; in the case of the adjunct professor, findings indicated that little or no training relative to working with students identified with autism had been provided by the community college. While research 
indicated that additional training to address the needs of students identified with autism would be welcomed by faculty (Casebolt \& Hodge, 2010; McKeon, Alpern, \& Zager, 2013) a recent study indicates that $40.6 \%$ of new faculty were not provided with inservice training relative to students with ASD (Brown, 2012). Given that adjunct professors represent $70 \%$ of new hires at community colleges (Center for Community College Student Engagement, 2014), the impact of lack of training of part time faculty is staggering.

The picture presented by these three professors is an abysmal one; these teachers had little desire to accommodate neurodiversity or had no training as to how to do so. Whether this was representative of the teaching staff in general is unknown, but the likelihood that these findings are wholly aberrational to the general tenor of the staff is improbable.

The implication was unambiguous; community college administration needs to adopt as a strategic target the training of all staff as to the nuances of autism, the supports and accommodations available and expected within the community college classrooms, and mechanisms for validating and affirming these students. Inclusive approaches would likely hold the greatest promise for supporting all students in an environment that has the least potential for othering. Researchers should explore strategies for providing such training and fostering inclusive settings within the community college environment.

Foundational Element Summary. The emerging picture of the community college was mixed; though a plurality of students reported positive feelings about the school, others reported feelings of disrespect. DSPS coordinators expressed confidence in their staff and the greater faculty, but students reported incidents of staff "othering". 
Faculty self-reported either a lack of training or attitudes inconsistent with the needs of students identified with autism, the stated intents of the college, and applicable law. Data received from DSPS leadership indicated that they are confused about the neurodiverse and how best to provide services for these students.

As in the case of the secondary school, the student identified with autism might be sufficiently fortuitous to successfully navigate the community college experience, yet the architecture and culture as analyzed indicated an environment where success was far from assured. To facilitate success, the community college needs to enhance their validation efforts in accordance with the views of Rendón (1994), provide school-wide training to ensure that all staff and faculty understand the neurodiverse and how best to support their collegiate efforts, and foster an inclusive environment for all.

\section{Student Struggles and Affirmations}

A critical factor that affected most of the students participating in this study is that they experienced some degree of anxiety associated with graduation and what awaited them afterwards. This anxiety can be viewed from the perspective of the theory of loss aversion proffered by Morrison and Clark (2016) and the research conducted by Browning, Osborne and Reed (2009). Loss aversion theory states that individuals “migrate" towards a state of increased stability; Browning, Osborne and Reed (2009) reported that as students with ASD approached the conclusion of their secondary school experience, they perceived themselves to be less able to deal with stress and were unable to identify the reasons why. This inability to deal with stress and the anxiety reported in this study may well be two symptoms of a root cause; the uncertainty that the loss of their secondary school stability and the transition to an unknown future engendered. Indeed, 
transition from secondary school to a postsecondary environment might well be viewed as the antithesis of migration towards stability; it is rather a forced migration towards an often-uncertain future.

Addressing this anxiety should begin at home; parents need to work with their children to normalize these feelings of anxiety as well as provide strategies for coping with these anxieties. Hodgson, Freeston, Honey, and Rodgers (2017) reported that anxiety is a common issue with students with ASD and conducted a study wherein strategies utilized by parents to address anxiety were reported. These strategies involved familiarization and repetition to increase certainty about an event as well as actively incorporating uncertainty into their child's routine to encourage tolerance of uncertainty. Although the children studied were between 8 and 14 years old, age-appropriate modifications of these strategies could prove useful to parents in normalizing their children's transition out of secondary school.

Secondary school staff can incorporate normalization strategies similar to those reported by Hodgson et al. (2017) into the recommended school sociocommunicative transition architecture; researchers can provide support through identification and ongoing study of best practices relative to normalizing and coping with transition anxiety.

Beyond anxiety, transitioning students reported concerns over sensory issues and sociocommunicative challenges. Challenges related to these concerns have been previously reported in the literature (Adreon \& Durocher, 2007; Gillot \& Standen, 2007). For the participating students, concerns regarding making friends or communicating were often addressed by limiting time on campus or pursuing environments where social interaction was discouraged. Sensory concerns were reported as causing discomfort and 
were addressed through social endurance. These mechanisms for coping with sociocommunicative and sensory challenges would likely foster a dissociation with the community college, thereby promoting desistance.

Community college practitioners and administration can respond to these concerns through an increased awareness of these challenges. Ensuring that postsecondary classrooms are inclusive, welcoming environments is a somewhat thorny issue; no research has been reported relative to best practices regarding this matter and what research has been conducted into inclusive environments for students identified with autism focuses on elementary classrooms and was inconclusive (Martin, 2016). Still, individual classroom teachers can do much to mitigate sensory and sociocommunicative challenges through personal affirmation and validation consistent with the recommendations of Rendón (1994).

This previous recommendation aligned with a critical factor reported by the majority of students participating in the study; they experienced positive interactions with their teachers and/or the disability services office. For some, these experiences were a source of personal satisfaction. This satisfaction seemed to reinforce the views of Rendón (1994), whose theory of validation calls for intentional, proactive affirmation to promote social acclimation, though no explicit evidence from the study pointed to community college staff intentionally affirming the students.

While evidence here presented supports the existence of a nurturing environment for students, other evidence within the study shows that validation was likely erratic at best. To promote consistent, affirming interaction, DSPS staff and faculty should receive ongoing training to foster positive, validating engagement with all students, including 
those identified with autism. This training should include part-time and student staff. Though applicable research is scant, Austin (2013) reported that among elementary school paraeducators, training relative to students with disabilities was often insufficient. Layden et al. (2018) reported improved overall knowledge by K-12 paraprofessionals who participated in a five-module online training focused on ASD. A similar, ageappropriate online course would provide an equivalent baseline of training in a convenient format for all staff.

Foundational Element Summary. It was clear from a review of the findings of the experiences and affirmations of participating students that much of what was perceived as a challenge was socially driven. Students experienced anxiety as well as social and sensory challenges through the transition process. These challenges served to inhibit rather than promote transition. To address the anxiety and sociocommunicative variances, students identified with autism who pursue community college need to address their anxiety through strategies for increasing certainty and/or normalizing uncertainty. They need to be provided with mechanisms for coping, be assured of their viability as a transitioning student and welcomed into an inclusive environment that validates all students. Such an approach should foster a successful transition and lead to an environment that would provide the proactive affirmation needed while minimizing stigma and othering.

\section{The Essential Nature of Personal Support}

Analysis of the data resulted in the emergence of a critical foundational element: regardless of the source, personal support from a "familiar" was critical for successful transition from secondary school to community college. Students who evidenced strong 
personal support from a "familiar" universally transitioned to community college and were still in attendance at the conclusion of the study; students who did not evidence support from a "familiar" did not complete the study.

Parents. Of the various "familiars" utilized, parents were the most often cited and had the most sweeping impact. Secondary school professionals noted the privileged role parents hold in acting on behalf of their children to facilitate a successful transition; they met with professionals, gathered information and ensured that all processes were accomplished. They further posited that unlike the neurotypical child, parental involvement for a student identified with autism needs to increase after secondary school graduation. Parents expressed their concerns over the uncertainty of the transition process and how important it was to them to make sure their children successfully made the adjustment. Students noted how parents utilized their position to influence and sometimes compel them to attend a postsecondary institution. Once community college attendance began, parents were further involved; they may pay for college, provide transportation to and from the institution, and tutor their child as well.

Research supported the importance of parents in the transition process. Mitchell and Beresford (2014) reported that parents can serve as important support mechanisms for the educational transitioning of their adult children. Giarelli et al. (2013) identified parents as potential "mentors" through the transition process. Chiang et al. (2012) included in their recommendations that the secondary school develop and maintain an ongoing partnership with parents of students with IEPs. Peña and Kocur (2013) reported that parents continued to aid their children with ASD while at community college through supporting students in navigating campus services and encouraging independence. Ebel 
(2015), posited that parents of students with ASD needed to maintain communication while providing ongoing advocacy and support throughout the postsecondary experience.

Parents hold a robust position of privilege relative to serving as a "familiar" for their children. Children have been reliant upon their parents since birth; while graduating from secondary school and turning eighteen are both symbolic and legal hallmarks of adulthood for the student, the psychology of parent-child interaction likely does not change so abruptly. The parents may continue to view their child as benefitting from their guidance and support, and the child may be willing to accept it. In addition, parents were still viewed by the secondary school professionals as legitimate advocates for their children throughout their attendance, regardless of when they turned eighteen. Because of this, community college outreach personnel would also be obliged to accept parents on these terms while in the secondary school environment. This status held by parents established them as preeminent in terms of direct support for their children; they must strive to leverage their privilege to ensure that their child receives all the support and guidance they need. Secondary school and community college personnel need to understand the value of the parent as an asset in facilitating a successful student transition and should nurture continuing parental guidance and support.

Extended Family and Peers. The role of "familiar" may be filled by members of the student's extended family or peers, who provide essential support, though in less direct ways. Often "guidance" provided by extended family and peers manifested itself as role models; they provided an example that the student could follow in molding their own lives. Whether the role model was an aunt who is a nurse, a cousin who is experiencing success at college, or a brother on the same path, these individuals, by their 
example, telegraphed to the student that they too can take this path and be successful. The inference was that by knowing these individuals personally, the student identified with autism could view themselves as equally capable of pursuing the same course.

Extended family and peers can also provide emotional support and/or guidance. This support and guidance can take a variety of forms. For some, it is practical advice; for others, emotional support. Regardless of the form this guidance and support takes, it plays a key role in reinforcing the impetus to succeed at the community college.

Research has shown the efficacy of role models in aiding students with ASD (Locke, Rotheram-Fuller, \& Kasari, 2012; Mathews, Vatland, Lugo, Koenig, \& Gilroy, 2018). Cochran (2009) reported that a peer-mentor can have the benefit of reducing stress during transition, Koegel, Ashbaugh, Koegel, Detar \& Regester (2013) advised that socialization mentors can aid in acclimating to community college, and Kelly and Joseph (n.d.). recommended maximizing social supports and peer assistance.

For students and parents, ensuring that extended family and peers were available to provide role models and emotional support was not something one can simply employ. Students either had extended families that provide positive role models, or they didn't; maintaining robust social relationships with peers is often a challenge for the neurodiverse, especially once the shared routine of secondary school ends.

This challenge of maintaining relationships that serve as role models or otherwise positively impact transition can be addressed through the social media mechanisms suggested for use by the secondary school. Social media platforms could allow students to continue secondary school relationships in a way that might provide a degree of the support and guidance necessary to facilitate a successful transition. Researchers might 
consider exploring how social media could best be utilized for this purpose, as well as what other strategies could be effective in maintaining peer relationships.

Research has offered that a "buddy system" or "mentor" approach might be of use to students in making the transition to community college (Barnhart, 2016; Cochran, 2009; Dillon, 2007; Giarelli et al., 2013; Kelly \& Joseph, n.d.; Koegel, Ashbaugh, Koegel, Detar \& Regester, 2013; Neville \& White, 2011). This approach can be both beneficial and limiting; a programmatic, structured approach aligning with the recommendations outlined in research can provide the support and guidance necessary to facilitate transition, but the forced nature of the approach limited the likelihood that the mentor and mentee will develop the strong bond needed to ensure a robust mentorship, while likely ensuring the stigmatization of the mentee as somehow deficient in needing an assigned mentor. This does not mean that the mentor approach is invalid. Still, the benefits of a mentor are limited by the programmatic nature of the offering. Where the mentor can be most effective is in providing accurate, unbiased guidance, not encumbered by the complexities that a personal relationship entails.

Of the nine students who participated in the study, those who did not articulate that they had a "familiar" to rely upon failed to complete the study; given that failure to complete the study may have evidenced some degree of academic desistance as well, the benefit of a robust personal support mechanism is likely critical. Further research should focus on how best to implement a "mentor" program for the transition process. More studies involving larger and more socioeconomically and ethnically diverse populations should be undertaken to heighten our understanding of the role an assigned mentor can 
play in supporting transition for students identified with autism and how to implement such programs with a minimum of stigma.

Foundational Element Summary. Students identified with autism often experience anxiety and sociocommunicative challenges in navigating the transition from secondary school to community college. These challenges within the collegiate environment can lead to loneliness, depression and isolation (Gelbar, Smith, \& Reichow, 2014). Having a "familiar" that can provide guidance and support, whether it is a parent, member of the extended family, peer or assigned mentor would likely encourage both a less stressful and a more successful transition to community college.

\section{A Foundational Bricolage of Elements}

Utilizing the empowerment approach as a mechanism to leverage both medical model data and social model personal mindset, the researcher coaxed the emergence of five foundational elements that form a bricolage of transition from secondary school to community college for students identified with autism. These five foundational elements are: secondary school architecture and culture, challenges inherent in the summer "gap", community college architecture and culture, student struggles and affirmations and personal support from a "familiar"

These five components each work to promote or inhibit, to a greater or lesser extent, the transition from secondary school to community college. As each component was urging or restraining movement towards a successful transition, often concurrently with other components, the structure functioned in "dynamic tension" with itself to create the unified transition experience. To understand the transition for each student, and by understanding, be able to authentically provide guidance and support to ensure a 
successful transition, one must look at the various factors in play and evaluate to what extent a factor is working to promote or inhibit their transition. If a foundational component is inhibiting transition, it may be compensated for by other elements; in this manner, the foundational bricolage shares conceptually with Schlossberg's Transition Theory (Lee et al., 2014). Viewed in total, the bricolage can be viewed as either promoting or inhibiting transition dependent on its individual student effect.

\section{A Missing Element}

It is not only important to note what elements form the bricolage, but also which elements were absent; though the students report heavy emphasis on the self-advocacy and sociocommunicative challenges faced, neither the secondary school nor the community college evidenced any programmatic mechanism for addressing these challenges. Through accommodations such as private testing settings and more time, some effort was informally made to ameliorate anxiety; though temporarily beneficial, greater effort needs to be made to provide settings that address social variances and empower students. An integrated, inclusive approach that provides sociocommunicative and self-advocacy strategies for all students, thus minimizing othering, should be the aim of both the secondary school and community college.

\section{Limitations}

This dissertation contributes to the literature on transition from secondary school to community college for students identified with autism. However, a convenience-based population, sociocommunicative challenges faced by some of the students, students who chose not to enroll in the fall, and the comparatively short timeframe of the study created limitations. 
First, the schools studied, and the population interviewed were largely convenience-based; utilizing personal contacts within the participating secondary school provided access to staff and administration at that school and through those contacts, the participating community college. Therefore, this study revolved around a single community college and its feeder high school. A total of 26 individuals participated in the study. The demographics of this study are found in Appendix D. Results of this study should not be generalized.

Second, as reported in this study, students identified with autism often experience sociocommunicative challenges. This was experienced within the context of the student interviews. Several of the students were highly reserved and responded to questions using only a few words. This prompted careful prodding to obtain richer responses. Not wanting to inadvertently lead the student, such prodding was measured and occasionally resulted in richer responses not being forthcoming. As a result, some students are less likely to be fully represented in the findings.

Third, of the nine students who participated in the study, two chose not to enroll in the fall semester. No information was obtainable regarding their reasons for not enrolling. In addition, one student reported that they were delaying enrollment until Spring 2019. The actions of these students resulted in three instances of incomplete student data.

Fourth, though matters of race, gender and sexuality have the potential to impact experiences such as transition, these aspects of participant being were not the focus of this study and were not evaluated relative to their potential impact on study findings. 
Fifth, the student interviews were conducted over a relatively short period from the late spring through the early fall of 2018. The final interview was conducted early in the first semester of college attendance. It is uncertain whether this early interview had an impact on student views of the community college, but additional interviews later in the semester would have provided additional depth to the data collected.

Though rich data was collected which led to significant findings and pointed towards other potentially noteworthy data, the limitations inherent in this study constrain its generalizability. For this reason, additional research on this topic is recommended. The specifics of this recommended research are contained in the following section.

\section{Recommendations for Future Research}

This dissertation provides noteworthy contributions to the current literature on transition. Prior research on transition from high school to postsecondary institutions had focused on either the secondary school or the college; scant research had been conducted from a unified perspective. Still, this study was conducted in a limited fashion; as such, it both focused on the need for unified research and highlighted areas for future consideration.

Secondary school case managers oversee students identified with autism within several settings based on classifications that, as stated before, can be somewhat arbitrary. Removing the stigma of such classifications and promoting an inclusive educational environment that prepares all students for a successful transition should be the goal of secondary schools. Research into secondary schools that incorporate such inclusive mechanisms and the challenges that may arise would be beneficial in better understanding how such an approach can be fostered. 
Throughout the transition period, the summer "gap" presented the greatest challenge to overcome. Secondary school had abruptly ended, support mechanisms had generally disappeared, and the students found themselves "at loose ends", waiting for the start of college. What happens during this period that results in some students who have affirmed their intent to attend college desisting in that goal? What could be done to better incorporate that time into a seamless flow from high school to college? Research addressing these concerns would be helpful in answering these questions, thereby providing tools to assist in navigating the summer break.

Findings demonstrated that students who transitioned from high school to community college experienced challenges that were primarily socially driven; they experienced heightened anxiety, sociocommunicative challenges and sensory issues, all impacting social interaction and assimilation. The participating secondary school presented a robust transition program, yet no evidence was presented indicating that the school addressed any socially driven challenges within the transition program. Whether socially driven challenges were not addressed by the school, addressed informally within individual school settings, or addressed within another context is unknown. Research directed towards understanding the mechanisms in play by the school to ensure socially driven challenges are addressed would be beneficial.

Finally, additional research into transition for students identified with autism needs to be conducted at other sites and in other environments to begin to develop a model that can be broadly utilized. Data relative to participant race, gender and sexuality should be collected with the intent of evaluating the impact of states of participant being relative to the transition experience. Future studies should utilize multiple data collection 
methods such as interviews, surveys and focus groups. Additionally, the research should be conducted beginning in the fall of the senior year and continuing through at least the end of the first semester of community college to better evaluate the impact of transition mechanisms as well as desistance.

\section{Conclusion}

This dissertation study explored and investigated the experiences and perspectives of students identified with autism, their parents/caregivers, faculty/staff, and other key parties as they transitioned from secondary school to community college. The findings generated a preliminary understanding of the mechanisms that impact transition, identified potential factors that can be leveraged to promote a successful transition, and provided indications of why so few who espouse an intent to pursue postsecondary education engage or persist in that endeavor.

One of the most significant findings to emerge from this study was the emergence of a preliminary model of transition whereby students identified with autism experience transition within the bricolage of five foundational elements. These elements are (1) the culture and architecture of the secondary school, (2) the hazards associated with the summer "gap", (3) the culture and architecture of the community college, (4) the struggles and affirmations experienced by the individual student, and (5) the essential nature of support from a "familiar". These foundational elements individually serve to either promote or inhibit a successful transition, and together they function in "dynamic tension" to collectively encourage or discourage transition.

A second finding of nearly equal significance was that although secondary and postsecondary institutions evidence rigorous mechanisms to aid in transition, these 
mechanisms were heavily reliant on the individual actions of staff and could be - and sometimes were $=$ defeated by staff. Associated with this finding was that administrative staff often articulated perceptions of the culture of the institution that were not always reflected by the faculty or staff of that institution.

A third finding was that students identified with autism may benefit from widely disparate service mechanisms while in attendance at secondary school due to a convoluted structure of diagnosis and categorical assignment. This medical approach resulted in neurodiverse students receiving services that may or may not have benefitted their individual variances.

A fourth finding was that students identified with autism generally expressed little reservations as to their academic capability to perform at the postsecondary level, but evidenced anxiety over a "forced migration" and significant social challenges. These challenges can be ameliorated through the support of a "familiar" throughout the process and the validation of the community college faculty and staff.

A fifth finding was that the summer "gap" represents a critical and potentially hazardous period for students identified with autism and their successful transition. The abrupt end to support and the unstructured nature of the period can result in students finding themselves adrift and without direction. For some, the findings suggested that this lack of direction resulted in a derailment of the transition process.

Finally, the heavy emphasis by the secondary school on four-year institutions, resulting in "faux" enrollment being reported, coupled with the potential lack of direction evidenced during the summer "gap", may provide some tentative understanding of why 
so many students who report while in secondary school intentions to pursue postsecondary education, desist in the endeavor.

Findings from this study provide opportunities for students to understand and normalize their challenges, parents to take a greater role in supporting their children, practitioners to institute courses of action that nurture and promote inclusive educational strategies, and policymakers to institute changes that can have a positive impact on transition success. While this study has its limitations, it serves as a starting point; additional studies need to be conducted to more fully understand transition secondary school to community college for students identified with autism. 


\section{Appendix A}

\section{Institutional Review Board (IRB) Approvals}

\section{$\triangle \mathrm{CHAPMAN}$ UNIVERSITY \\ INSTITUTIONAL REVIEW BOARD}

SCOT DANFORTH

October 4, 2017

EDUCATION STUDIES

RE: CHAPMAN UNIVERSITY IRB\# 1718H041 How do students within the autism spectrum and other stakeholders view the services offered by the California community college's Disabled Students Programs and Services (DSPS) office in aiding their transition and impacting their success?

The above-referenced human-subjects research project has been approved by the Chapman University Institutional Review Board (CU IRB). This approval is limited to the activities described in the approved Protocol Narrative, and extends to the performance of these activities at each respective site identified in the Application for IRB Review. In accordance with this approval, the specific conditions for the conduct of this research are listed below, and informed consent from subjects must be obtained unless otherwise indicated below. Additional conditions for the general conduct of human-subjects research are detailed on the attached sheet.

NOTE: Approval by the Institutional Review Board does not, in and of itself, constitute approval for the implementation of this research. Other institutional clearances and approvals may be required (e.g., Institutional Biosafety Committee, Financial Conflict of Interest Oversight Committee, etc.). Research undertaken in conjunction with outside entities, such as drug or device companies, are typically contractual in nature and require an agreement between the University and the entity. Such device companies, are typicaly by an institutional official in Sponsored Programs Administration, a division in the Office of agreements must bexisty is not obligated to legally defend or indemnify an employee who individually enters into these agreements and investigators are personally liable for contracts they sign. Accordingly, the project should not begin until all required approvals have been obtained.

Questions concerning the approval of this research project may be directed to the Office of Research, Chapman University, One University Drive, Orange, California 92866, or by calling 714-628-2833.

Expedited Review: 6,7

Natalie Del Rio IRB/FCOI Administrator

Approval Issued: 10/04/2017

Expiration Date: 10/03/2018

IRB Determinations as Conditions of Approval: Informed Consent Determinations:

1. Signed Informed Consent Required 


\title{
\begin{tabular}{c|c} 
CHAPMAN & Institutional REView BOARD
\end{tabular}
}

\author{
APPROVAL CONDITIONS FOR ALL CHAPMAN UNIVERSITY HUMAN RESEARCH PROTOCOLS
}

The Principal Investigator of the study is ultimately responsible for assuring all study team members adhere to applicable policies for the conduct of human-subjects research.

Principal Investigators are responsible for the retention of protocol-related records.

PROTOCOL EXPIRATION:

The Chapman University IRB approval letter references the protocol expiration date under the IRB Chair's signature authorization. A courtesy email will be sent approximately 60 to 90 days prior to expiration reminding the Principal Investigator to apply for continuing review. For studies granted Extended IRB Approval, a courtesy e-mail will be sent annually to verify eligibility for the continuation of extended approval. It is the Principal Investigator's responsibility to apply for continuing review and in order to ensure continuing approval throughout the conduct of the study. Lapses in approval must be avoided to protect the safety and welfare of enrolled subjects.

MODIFICATIONS \& AMENDMENTS:

No changes are permissible to the approved protocol or the approved, stamped consent form without the prior review and approval of the Chapman University IRB. All changes (e.g., a change in procedure, number of subjects, personnel, study locations, new recruitment materials, study instruments, etc.) must be prospectively reviewed and approved by the IRB before they are implemented.

APPROVED VERSIONS OF CONSENT DOCUMENTS, INCLUDING STUDY INFORMATION SHEETS:

Unless a waiver of informed consent is granted by the IRB, the consent documents (consent form; study information sheet) with the Chapman University IRB approval stamp must be used for consenting all human subjects enrolled in this study. Only the current approved version of the consent documents may be used to consent subjects. Approved consent documents are not to be used beyond the expiration date provided on the IRB approval letter.

ADVERSE EVENT \& UNANTICIPATED PROBLEMS REPORTING:

All unanticipated problem involving risk to subjects or others or serious adverse events must be reported to the Chapman University IRB in accordance with Federal regulations and Chapman University policy.

CHANGES IN FINANCIAL INTEREST:

Any changes in the financial relationship between the study sponsor and any of the investigators on the study and/or any new potential conflicts of interest must be reported immediately to the Chapman University Financial Conflict of Interest Oversight Committee (FCOI). If these changes affect the conduct of the study or result in a change in the text of the currently-approved informed consent document, these changes must also be reported to the Chapman University IRB via a modification request. Research subject to FCOI oversight is not eligible for Extended IRB Approval.

CLOSING REPORT:

A closing report should be filed with the Chapman University IRB when the research concludes. 


\section{\begin{tabular}{l|l} 
CHA CHAMAN & INSTITUTIONAL REVIEW BOARD
\end{tabular}}

September 28, 2018

\section{PI: Scot Danforth \\ RE: Continuation Approval \\ IRB\#: 1718H041}

TITLE: How do students within the autism spectrum and other stakeholders view the services offered by the California community colleges...

The above-referenced human-subjects research project has been approved by the Chapman University Institutional Review Board (CU IRB). This approval is limited to the activities described in the approved Protocol Narrative and extends to the performance of these activities at each respective site identified in the Application for IRB Review. In accordance with this approval, the specific conditions for the conduct of this research are listed below, and informed consent from subjects must be obtained unless otherwise indicated below. Additional conditions for the general conduct of human-subjects research are detailed on the attached sheet.

NOTE: Approval by the Institutional Review Board does not, in and of itself, constitute approval for the implementation of this research. Other institutional clearances and approvals may be required (e.g., Institutional Biosafety Committee or Biosafety Officer, other EH\&S review by the Radiation Safety and/or Chemical Hygiene Officer, Risk Management, Financial Conflict of Interest Oversight Committee, etc.). Research undertaken in conjunction with outside entities, such as drug or device companies, are typically contractual in nature and require an agreement between the University and the entity. Such agreements must be executed by an institutional official in Sponsored Programs Administration, a division in the Office of Research. The University is not obligated to legally defend or indemnify an employee who individually enters into these agreements and investigators are personally liable for contracts they sign. Accordingly, the project should not begin until all required approvals have been obtained.

This research received review for the following reasons (where applicable):

- 6. Collection of data from voice, video, digital, or image recordings made for research purposes.

7. Research on individual or group characteristics or behavior (including, but not limited to, research on perception, cognition, motivation, identity, language, communication, cultural beliefs or practices, and social behavior) or research employing survey, interview, oral history, focus group, program evaluation, human factors evaluation, or quality assurance methodologies. 
In accordance with this approval, the specific conditions for the conduct of this research are listed below, and informed consent from subjects must be obtained unless otherwise indicated below.

Please ensure when obtaining Informed Consent that the IRB Stamped PDF Version is used. Stamped Consent Documents can be found within the Attachments Tab on the Study Detail level of your approved Study.

For Approval Conditions please visit Chapman University Approval Conditions tab.

Questions concerning the approval of this research project may be directed to the Office of Research, Chapman University, One University Drive, Orange, California 92866, or by calling 714-628-2833.

Thank you.

Natalie Del Rio

IRB/FCOI Administrator

APPROVAL ISSUED: September 18, 2018

EXPIRATION DATE: September 17, 2019

FWA\#00011020

cc: Chapman University IRB; Douglas Highlen 
September 28, 2018

Scot Danforth

RE: Modification Approval

IRB\#: 1718H041

How do students within the autism spectrum and other stakeholders view the services offered by the California community colleges...

The following modification(s) for the human subjects research protocol referenced above has/have been approved by the Chapman University Institutional Review Board (CUIRB). Below is a summary of the approved changes requested:

\section{Other Changes:}

- $\quad$ As Pilot Study ensured, it was determined that additional data from multiple sources and multiple strategies for obtaining such data could better form a bricolage of understanding relative to the experience of students identified with autism as they transitioned to high school to community college. Essentially, data from the pilot study is being incorporated into a larger and more diverse data set for the purposes of the dissertation.

${ }^{* *}$ The IRB may not have approved all changes proposed in the modification request. Review the above summary of approved changes and any revised documents provided with this letter. If a requested change does not appear in the summary or in the revised documents, the IRB did not approve that change. Please consult with the IRB staff for further information. Changes to approved protocols may not be made without prior approval by the IRB.

Note: Please discontinue use of any previous versions of the informed consent document and use only the most updated version for enrollment of all new subjects. Questions concerning approval of this study may be directed to the Office of Research and Sponsored Programs Administration, Chapman University, 714-628-2833, irb@chapman.edu. 
Thank you.

Julia Boehm, PhD

Chair, Institutional Review Board

APPROVAL ISSUED: September 18, 2018

EXPIRATION DATE: September 17, 2019

FWA\#00011020

cc: IRB Office 


\title{
Appendix B \\ Consent Form \\ CHAPMAN UNIVERSITY \\ CONSENT TO ACT AS A HUMAN RESEARCH SUBJECT
}

TITLE OF STUDY: What are the critical factors that impact college trajectory students identified with autism as they transition from a secondary school to a California community college and how do these students and other stakeholders experience this transition?

You are being asked to participate in a research study. Participation is completely voluntary. Please read the information below and ask questions about anything that you do not understand. A researcher listed below will be available to answer your questions.

\author{
RESEARCH TEAM \\ Lead Researcher \\ Douglas Highlen, MA, Doctoral Student \\ College of Educational Studies \\ 714 280-1488 highl104@mail.chapman.edu
}

Faculty Sponsor

Scot Danforth, PhD

College of Educational Studies

$714744-7674$

danforth@chapman.edu

STUDY LOCATION(S): TBD

STUDY SPONSOR(S): Chapman University 


\section{Investigator Financial Conflict of Interest}

No one on the study team has a disclosable financial interest related to this research project.

\section{WHY IS THIS RESEARCH STUDY BEING DONE?}

The purpose of this research study is to identify and understand the critical factors that impact college trajectory students identified with autism as they transition from a secondary school to a California community college and how do these students and other stakeholders experience this transition.

\section{HOW MANY PEOPLE WILL TAKE PART IN THIS STUDY?}

We expect twenty to twenty-five people will be in this research study. All study procedures will be done at a location agreeable to both participant and researcher. Some interviews may, of necessity, be conducted by telephone.

\section{WHAT PROCEDURES ARE INVOLVED WITH THIS STUDY AND HOW LONG WILL THEY TAKE?}

1. Each participant will undergo a series of interviews. Interviews will be audio recorded. The semi-structured interview will gather an understanding of your views, perceptions and experiences surrounding transition from secondary school to a California community college. The interview protocol will be flexible (approximately 12 open-ended questions) to allow for the questions to evolve throughout the study and will not be provided in advance.

2. Participation in the study will include about two to three interviews and take a total of about two hours over a period of twenty weeks.

\section{AM I ELIGIBLE TO PARTICIPATE IN THIS STUDY?}

Please note this may not be a complete list of eligibility criteria. We have included a few examples of study criteria to help you better understand how your eligibility in the study will be determined; your study team will go through the study eligibility criteria with you to verify if you qualify for participation in this study.

\section{Inclusion Requirements}

You can participate in this study if you are

- 18 years of age or older

- $\quad$ Identified with autism

- $\quad$ intending to attend or currently attending a California community college 
- $\quad$ the caregiver/parent of a qualifying individual.

- $\quad$ Other key participant in the transition process (i.e., high school counselor, college counselor, etc.)

\section{Exclusion Requirements}

You cannot participate in this study if you are

- $\quad$ under 18 eighteen years of age

- not identified with autism,

- $\quad$ not intending to attend or attending community college

- $\quad$ not the parent/caregiver of a qualifying individual.

- $\quad$ Not a stakeholder in the transition process (i.e., high school counselor, college counselor, etc.)

\section{WHAT ARE THE POSSIBLE DISCOMFORTS OR RISKS RELATED TO THE STUDY?}

There are no known harms or discomforts associated with this study beyond those encountered in normal daily life. The possible risks and/or discomforts associated with the procedures described in this study include: anxiety, embarrassment, social stigma (shame or disgrace); and invasion of privacy.

Breach of Privacy and Confidentiality: As with any study involving collection of data, there is the possibility of breach of confidentiality of data. Every precaution will be taken to secure participants' personal information to ensure confidentiality.

\section{ARE THERE BENEFITS TO TAKING PART IN THE STUDY?}

\section{Participant Benefits}

You will not directly benefit from participation in this study.

\section{Benefits to Others or Society}

Your responses may assist researchers studying students identified with autism and services offered at California high schools and community colleges and the results may be disseminated in a way that benefits education in general.

\section{WILL I BE PAID FOR TAKING PART IN THIS STUDY?}

\section{Compensation}

You will not be compensated for your participation in this research study. 


\section{Reimbursement}

You will not be reimbursed for any out of pocket expenses, such as parking or transportation fees.

\section{WHAT HAPPENS IF I WANT TO STOP TAKING PART IN THIS STUDY?}

If you elect to withdraw or are withdrawn from this research study, the researchers will discuss with you what they intend to do with your study data. Researchers may choose to analyze the study data already collected or they may choose to exclude your data from the analysis of study data and destroy it, as per your request.

\section{HOW WILL MY PERSONAL INFORMATION BE KEPT?}

\section{Subject Identifiable Data}

Identifiable information collected about you will be removed at the end of data collection.

\section{Data Storage}

Research data will be stored electronically on a laptop computer in an encrypted file and is password protected. The audio recordings will also be stored in a secure location; then transcribed and erased at the end of the study.

\section{Data Retention}

The researchers intend to keep the research data until the research is published and/or presented.

\section{WHO WILL HAVE ACCESS TO MY STUDY DATA?}

The research team, authorized Chapman University personnel, and regulatory entities such as the Office of Human Research Protections (OHRP), may have access to your study records to protect your safety and welfare.

Any information derived from this research project that personally identifies you will not be voluntarily released or disclosed by these entities without your separate consent, except as specifically required by law. Study records provided to authorized, non- 
Chapman University entities will not contain identifiable information about you; nor will any publications and/or presentations without your separate consent.

While the research team will make every effort to keep your personal information confidential, it is possible that an unauthorized person might see it. We cannot guarantee total privacy.

\section{WHO CAN ANSWER MY QUESTIONS ABOUT THE STUDY?}

If you have questions, concerns, or complaints, or think the research has hurt you, talk to the research team at highl104@mail.chapman.edu

This research has been reviewed and approved by an Institutional Review Board ("IRB"). You may talk to them at 714-628-2833 or irb@chapman.edu if:

- Your questions, concerns, or complaints are not being answered by the research team.

- You cannot reach the research team.

- You want to talk to someone besides the research team.

- $\quad$ You have questions about your rights as a research participant.

- $\quad$ You want to get information or provide input about this research.

\section{HOW DO I AGREE TO PARTICIPATE IN THIS STUDY?}

You should not sign this consent form until all of your questions about this study have been answered by a member of the research team listed at the top of this form. You will be given a copy of this signed and dated consent form to keep. Participation in this study is voluntary. You may refuse to answer any question or discontinue your involvement at any time without penalty or loss of benefits to which you might otherwise be entitled. Your decision will not affect your future relationship with Chapman University. 


\section{AUDIO RECORDING:}

I have received an adequate description of the purpose and procedures for audiorecording sessions during the course of the proposed research. I give my consent to allow myself to be audio-recorded during participation in this study, and for those records to be reviewed by persons involved in the study, as well as for other professional purposes as described to me.

Yes, I agree to allow the research team to audio record my interview(s).

No, I do not wish to have my interview audio recorded.

VIDEO RECORDING: Not applicable

Your signature below indicates you have read the information in this consent form and have had a chance to ask any questions you have about this study.

I agree to participate in the study.

\section{Subject Signature}

\section{Date}

\section{Printed Name of Subject}




\section{Printed Name of Researcher}

\section{CHAPMAN UNIVERSITY}

\section{Experimental Subject's Bill of Rights}

\section{The rights listed below are the right of every individual asked to participate in a research study. You have the right:}

1. To be told about the nature and purpose of the study.

2. To be told about the procedures to be followed in the research study, and whether any of the drugs, devices, or procedures is different from what would be used in standard practice.

3. To receive a description of any side effects, discomforts, or risks that you can reasonably expect to occur during the study.

4. To be told of any benefits that you may reasonably expect from the participation in the study, if applicable.

5. To receive a description of any alternative procedures, drugs, or devices that might be helpful, and their risks and benefits compared to the proposed procedures, drugs or devices.

6. To be told of what sort of medical treatment, if any, will be available if any complications should arise.

7. To be given a chance to ask any questions concerning the research study both before agreeing to participate and at any time during the course of the study.

8. To refuse to participate in the research study. Participation is voluntary. You may refuse to answer any question or discontinue your involvement at any time without penalty or loss of benefits to which you might otherwise be entitled. Your decision will not affect your right to receive the care you would receive if you were not in the experiment. 
9. To receive a copy of the signed and dated written consent form and a copy of this form.

10. To be given the opportunity to freely decide whether or not to consent to the research study without any force, coercion, or undue influence.

If you have any concerns or questions regarding the research study, you should contact the research team listed at the top of the consent form. If you are unable to reach a member of the research team and have general questions, or you have concerns or complaints about the research study, research team, or questions about your rights as a research subject, please contact the Chapman University IRB staff at 714-628-2833 or irb@chapman.edu. 


\title{
Appendix C
}

\section{Supplemental Consent Form}

\section{CHAPMAN UNIVERSITY}

\section{CONSENT TO PROVIDE EXIT IEP AND ALLOW “SHADOW ON-CAMPUS TOUR”}

TITLE OF STUDY: What are the critical factors that impact college trajectory students identified with autism as they transition from a secondary school to a California community college and how do these students and other stakeholders experience this transition?

You are being asked to provide additional research data for the research study. Participation is completely voluntary. Choosing not to participate in the supplemental data collection is no way changes ones participation within the general study. Please read the information below and ask questions about anything that you do not understand. A researcher listed below will be available to answer your questions.

\author{
RESEARCH TEAM \\ Lead Researcher \\ Douglas Highlen, MA, Doctoral Student \\ College of Educational Studies \\ 714 280-1488 highl104@mail.chapman.edu
}

\author{
Faculty Sponsor \\ Scot Danforth, PhD \\ College of Educational Studies \\ $714744-7674$ \\ danforth@chapman.edu
}

STUDY LOCATION(S): TBD

STUDY SPONSOR(S): Chapman University 


\section{Investigator Financial Conflict of Interest}

No one on the study team has a disclosable financial interest related to this research project.

\section{WHY IS THIS RESEARCH STUDY BEING DONE?}

The purpose of this research study is to identify and understand the critical factors that impact college trajectory students identified with autism as they transition from a secondary school to a California community college and how do these students and other stakeholders experience this transition.

\section{HOW MANY PEOPLE WILL TAKE PART IN THIS STUDY?}

We expect twenty to twenty-five people will be in this research study.

\section{WHAT PROCEDURES ARE INVOLVED WITH THIS SUPPLEMENTAL CONSENT REQUEST AND HOW LONG WILL THEY TAKE?}

1, Each participant will be asked to provide a copy of the transition component of their exit IEP.

2, Each participant will be asked to permit the Lead Researcher to "shadow" them through an on-campus "tour", during which the Lead Researcher will conduct a "wandering semi-structured interview" to better understand the student experience relative to the campus and determine areas of student strength and challenges within the campus environment. Interviews will be audio recorded. The interview protocol will be flexible (approximately 8 open-ended questions) to allow for the questions to evolve throughout the study and will not be provided in advance. This "on-campus tour" should generally not exceed 30 minutes in length.

\section{AM I ELIGIBLE TO PARTICIPATE IN THIS SUPPLEMENTAL DATA COLLECTION?}

If you are a part of the ongoing study and have signed a general consent form, you are eligible to participate in the supplemental data collection.

\section{WHAT ARE THE POSSIBLE DISCOMFORTS OR RISKS RELATED TO THE SUPPLEMENTAL DATA COLLECTION?}

There are no known harms or discomforts associated with this study beyond those encountered in normal daily life. The possible risks and/or discomforts associated with 
the procedures described in this study include: anxiety, embarrassment, social stigma (shame or disgrace); and invasion of privacy.

Breach of Privacy and Confidentiality: As with any study involving collection of data, there is the possibility of breach of confidentiality of data. Every precaution will be taken to secure participants' personal information to ensure confidentiality.

\section{ARE THERE BENEFITS TO TAKING PART IN THE SUPPLEMENTAL DATA COLLECTION?}

\section{Participant Benefits}

You will not directly benefit from participation in this study.

\section{Benefits to Others or Society}

Your responses may assist researchers studying students identified with autism and services offered at California high schools and community colleges and the results may be disseminated in a way that benefits education in general.

\section{WILL I BE PAID FOR TAKING PART IN THIS SUPPLEMENTAL DATA COLLECTION?}

\section{Compensation}

You will not be compensated for your participation in this research study.

\section{Reimbursement}

You will not be reimbursed for any out of pocket expenses, such as parking or transportation fees.

\section{WHAT HAPPENS IF I WANT TO STOP TAKING PART IN THIS SUPPLEMENTAL DATA COLLECTION?}

If you elect to withdraw or are withdrawn from this supplemental data collection, the researchers will discuss with you what they intend to do with your study data. Researchers may choose to analyze the study data already collected or they may choose to exclude your data from the analysis of study data and destroy it, as per your request. 


\section{HOW WILL MY PERSONAL INFORMATION BE KEPT?}

\section{Subject Identifiable Data}

Identifiable information collected about you will be removed at the end of data collection.

\section{Data Storage}

Research data will be stored electronically on a laptop computer in an encrypted file and is password protected. The audio recordings will also be stored in a secure location; then transcribed and erased at the end of the study.

\section{Data Retention}

The researchers intend to keep the research data until the research is published and/or presented.

\section{WHO WILL HAVE ACCESS TO MY STUDY DATA?}

The research team, authorized Chapman University personnel, and regulatory entities such as the Office of Human Research Protections (OHRP), may have access to your study records to protect your safety and welfare.

Any information derived from this research project that personally identifies you will not be voluntarily released or disclosed by these entities without your separate consent, except as specifically required by law. Study records provided to authorized, nonChapman University entities will not contain identifiable information about you; nor will any publications and/or presentations without your separate consent.

While the research team will make every effort to keep your personal information confidential, it is possible that an unauthorized person might see it. We cannot guarantee total privacy.

\section{WHO CAN ANSWER MY QUESTIONS ABOUT THE SUPPLEMENTAL DATA COLLECTION?}

If you have questions, concerns, or complaints, or think the research has hurt you, talk to the research team at highl104@mail.chapman.edu 
This research has been reviewed and approved by an Institutional Review Board ("IRB"). You may talk to them at 714-628-2833 or irb@chapman.edu if:

- Your questions, concerns, or complaints are not being answered by the research team.

- You cannot reach the research team.

- You want to talk to someone besides the research team.

- $\quad$ You have questions about your rights as a research participant.

- $\quad$ You want to get information or provide input about this research.

\section{HOW DO I AGREE TO PARTICIPATE IN THIS SUPPLEMENTAL DATA COLLECTION?}

You should not sign this consent form until all of your questions about this study have been answered by a member of the research team listed at the top of this form. You will be given a copy of this signed and dated consent form to keep. Participation in this study is voluntary. You may refuse to answer any question or discontinue your involvement at any time without penalty or loss of benefits to which you might otherwise be entitled. Your decision will not affect your future relationship with Chapman University.

AUDIO RECORDING:
I have received an adequate description of the purpose and procedures for audio-
recording sessions during the course of the proposed research. I give my consent to
allow myself to be audio-recorded during participation in this study, and for those
records to be reviewed by persons involved in the study, as well as for other
professional purposes as described to me.
interview(s). Yes, I agree to allow the research team to audio record my
\[ \text { No, I do not wish to have my interview audio recorded. } \]
Signature of Participant


VIDEO RECORDING: Not applicable

Your signature below indicates you have read the information in this consent form and have had a chance to ask any questions you have about this study.

I agree to provide the transition component of the exit IEP to the researcher.

I agree to participate in a "shadow campus tour"

Subject Signature

Date

Printed Name of Subject

Researcher Signature

Date

Printed Name of Researcher

CHAPMAN UNIVERSITY

Experimental Subject's Bill of Rights

The rights listed below are the right of every individual asked to participate in a research study. You have the right: 
11. To be told about the nature and purpose of the study.

12. To be told about the procedures to be followed in the research study, and whether any of the drugs, devices, or procedures is different from what would be used in standard practice.

13. To receive a description of any side effects, discomforts, or risks that you can reasonably expect to occur during the study.

14. To be told of any benefits that you may reasonably expect from the participation in the study, if applicable.

15. To receive a description of any alternative procedures, drugs, or devices that might be helpful, and their risks and benefits compared to the proposed procedures, drugs or devices.

16. To be told of what sort of medical treatment, if any, will be available if any complications should arise.

17. To be given a chance to ask any questions concerning the research study both before agreeing to participate and at any time during the course of the study.

18. To refuse to participate in the research study. Participation is voluntary. You may refuse to answer any question or discontinue your involvement at any time without penalty or loss of benefits to which you might otherwise be entitled. Your decision will not affect your right to receive the care you would receive if you were not in the experiment.

19. To receive a copy of the signed and dated written consent form and a copy of this form.

20. To be given the opportunity to freely decide whether or not to consent to the research study without any force, coercion, or undue influence.

If you have any concerns or questions regarding the research study you should contact the research team listed at the top of the consent form. If you are unable to reach a member of the research team and have general questions, or you have concerns or complaints about the research study, research team, or questions about your rights as a research subject, please contact the Chapman University IRB staff at 714-628-2833 or irb@chapman.edu. 


\section{Appendix D}

\section{Summary of Participant Demographics}

Community College Personnel

\begin{tabular}{|l|l|l|l|l|}
\hline Participant & Pseudonym & Ethnicity/Gender & Age & Role \\
\hline CC1 & Linda Adams & CF & 62 & DSPS Director \\
\hline CC2 & Dr. Robert Miller & CM & 41 & DSPS Director \\
\hline CC3 & Barbara Wood & CF & 47 & Outreach Tech \\
\hline CC4 & Christine Wilson & CF & 66 & Instructor \\
\hline CC5 & Fred Richardson & CM & 58 & Instructor \\
\hline CC6 & Terry Edwards & CM & 31 & Instructor \\
\hline
\end{tabular}

High School Personnel

\begin{tabular}{|l|l|l|l|l|}
\hline Participant & Pseudonym & Ethnicity/Gender & Age & Role \\
\hline HS1 & Catherine Green & CF & 36 & Asst Principal \\
\hline HS2 & Stephanie Taylor & CF & 32 & Counselor \\
\hline HS3 & Doris Hall & CF & 42 & Gen Ed Instructor \\
\hline HS4 & Joe Rodriguez & H/LM & 34 & Sp Ed Instructor \\
\hline HS5 & Lois Perry & CF & 70 & Sp Ed Instructor \\
\hline HS6 & Larry Patterson & CM & 61 & Gen Ed Instructor \\
\hline
\end{tabular}


Parents

\begin{tabular}{|l|l|l|l|}
\hline Participant & Pseudonym & Ethnicity/Gender & Age \\
\hline P1 & James Williams & CM & 60 \\
\hline P2 & Bernadette Foster & CF & 56 \\
\hline P3 & Claire Williams & CF & 54 \\
\hline P4 & Mary Cooper & CF & 48 \\
\hline
\end{tabular}

\section{Students}

\begin{tabular}{|l|l|l|l|l|l|}
\hline Participant & Pseudonym & $\begin{array}{l}\text { Ethnicity/ } \\
\text { Gender }\end{array}$ & $\begin{array}{l}\text { Highest Parental } \\
\text { Education }\end{array}$ & $\begin{array}{l}\text { Household } \\
\text { Size }\end{array}$ & Age \\
\hline S1 & Fred Jackson & CM & HS Grad & 3 & 18 \\
\hline S2 & Steven Cooper & CM & College Grad & 5 & 19 \\
\hline S3 & $\begin{array}{l}\text { Briana } \\
\text { Williams }\end{array}$ & CF & Grad School & 4 & 18 \\
\hline S4 & Martin Diaz & H/LM & Some College & 6 & 18 \\
\hline S5 & Amy Martin & CM & College Grad & 2 & 18 \\
\hline S6 & $\begin{array}{l}\text { Kim } \\
\text { Hernandez }\end{array}$ & H/LF & Grad School & 3 & 18 \\
\hline S7 & Chris Jenkins & CM & Some College & 4 & 18 \\
\hline S8 & George Butler & OM & HS Grad & 5 & 19 \\
\hline S9 & $\begin{array}{l}\text { Raymond } \\
\text { Alcala }\end{array}$ & H/LM & Some HS & 4 & 18 \\
\hline S10 & & & & & \\
\hline
\end{tabular}

*Withdrew prior to first interview 


\section{Appendix E}

\section{Student Background Information}

DIRECTIONS: Please answer each question as accurately as possible by marking the correct answer.

1. Age

$\square 18$

$\square 19$

$\square 20$

2. Gender

$\square$ Male

$\square$ Female

Other

3. Household Size (Living at Home)

$\square 1$
$\square 2$
$\square 3$
$\square 4$
$\square 5$
$\square 6$
$\square 7$
$\square 8$
$\square$ Other

4. Student Ethnicity

$\square$ American Indian

$\square$ Asian

$\square$ Black

$\square$ Caucasian

$\square$ Hispanic/Latino

$\square$ Pacific Islander

Mixed Race

$\square$ Other

5. Father's Education

$\square$ Some High School

$\square$ High School Graduate

$\square$ Some College

$\square$ College Graduate

$\square$ Graduate School

$\square$ Professional School

6. Mother's Education

$\square$ Some High School

$\square$ High School Graduate

$\square$ Some College

College Graduate

$\square$ Graduate School

$\square$ Professional School 


\section{Appendix F}

\section{Semi-Structured Interview Guides}

\section{Interview Guide \#1 - Student}

Thank you for signing the consent form and agreeing to be part of my study on views and experiences with transition from high school and success at community college. Over the course of this interview I will be asking you a few questions. You are free to skip a question or not answer something at any point if it might be uncomfortable for you. Think of this as us having a conversation. Depending on your answers, I may ask additional questions to further explore your views.

1. Tell me about the experience of finishing and leaving high school.

2. How do you see the experience of planning to go to community college?

3. How do you see your preparation for this transition?

4. Tell me about the impact that your parents had on going to community college?

5. Tell me about the impact your friends had on going to community college?

6. Tell me about the impact the high school had on going to community college?

7. Tell me about the impact the community college had on choosing to go to community college?

8. Thinking about attending community college (next year), what do you think will be your areas of success?

9. Thinking about attending college (next year), what do you think will be your areas of needed support?

10. What else would you like to share about transitioning to community college?

Future Interview Topics/Questions:

- Continued experiences

- Successes

- Challenges

- Changes over time 
Interview Guide \#2 - Student

Thank you for agreeing to this second interview as part of my study on views and experiences with transition from high school and success at community college. Over the course of this interview I will be asking you a few questions. You are free to skip a question or not answer something at any point if it might be uncomfortable for you. Think of this as us having a conversation. Depending on your answers, I may ask additional questions to further explore your views.

1. Tell me about the experience of finishing and leaving high school.

2. Tell me about your plans for this fall?

3. How do you see your preparation for this transition?

4. Tell me about the impact that your parents had on this transition?

5. Tell me about the impact your friends had on this transition?

6. Tell me about the impact the high school had on this transition?

7. Tell me about the impact the community college had on this transition?

8. Thinking about (attending community college) (plans for the fall), what do you think will be your areas of success?

9. Thinking about (attending community college) (plans for the fall), what do you think will be your areas of needed support?

10. What else would you like to share about how things have changed since high school?

Future Interview Topics/Questions:

- Continued experiences

- Successes

- Challenges

- Changes over time 
Interview Guide \#3 - Student

Thank you for agreeing to this final interview as part of my study on views and experiences with transition from high school and success at community college. Over the course of this interview I will be asking you a few questions. You are free to skip a question or not answer something at any point if it might be uncomfortable for you. Think of this as us having a conversation.

Depending on your answers, I may ask additional questions to further explore your views.

1. Tell me about the experience of (beginning community college?) (this fall?)

2. How do you see your transition?

3. Tell me about the impact that your parents had on this transition?

4. Tell me about the impact your friends had on this transition?

5. Tell me about the impact the high school had on this transition?

6. Tell me about the impact the community college had on this transition?

7. Thinking about (attending community college) (plans for the fall), what do you think have been your areas of success?

8. Thinking about (attending community college) (plans for the fall), what have been your areas that needed support?

\section{For those who go to community college}

9. What autism related support have you received from the community college?

10. What has the community college done well?

11. Do you feel the school values you being there?

12. Do you feel the teachers are supportive of you?

13. How could the community college improve this support?

\section{For those who chose a different path}

9. When you were in high school, you thought you would go to community college... what changed your mind?

\section{Regardless of the path}

What else would you like to share about how things have changed since high school?

Thank you for participating in my study. 


\section{Interview Guide - Caregiver/Parent}

Thank you for agreeing to be part of my study on Caregiver/Parent views and experiences with transition and success. Over the course of about 30-45 minutes I will be asking you a few questions. You are free to skip a question or not answer something at any point if it might be uncomfortable for you. Think of this as us having a conversation. Depending on your answers, I may ask additional questions to further explore your views.

1. How do you view the experience of your son/daughter finishing high school?

2. How do you see the experience of your son/daughter transitioning to community college?

3. How do you see your son's/daughter's preparation for this transition?

4. Please share your experience of what transition assistance, if any, has been available for your son/daughter.

5. What challenges have you faced in obtaining the support your son/daughter needs?

6. Thinking about your son/daughter attending community college (next year), what do you anticipate being areas of success?

7. Thinking about your son/daughter attending community college (next year), what do you anticipate being areas where support will be needed?

8. Please share your understanding of what support is available for your son/daughter at community college?

9. What do you see as your role in this transition?

10. What else would you like to share regarding your son/daughter in transitioning to and succeeding in community college?

Thank you again for your time and responses.

Future Interview Topics/Questions:

- Continued experiences

- Successes

- Challenges

- Changes over time 
Interview Guide - Secondary school stakeholder

Thank you for agreeing to be part of my study on views and experiences relating to transition and success for students with autism. Over the course of about 30-45 minutes I will be asking you a few questions. You are free to skip a question or not answer something at any point if it might be uncomfortable for you. Think of this as us having a conversation. Depending on your answers, I may ask additional questions to further explore your views.

1. What is your role in transitioning students with autism from secondary school to college?

2. What is the process utilized to facilitate the successful transition of students with autism to college?

3. What stakeholders are involved in this process? (title not name)

4. What are the strengths of this process?

5. What improvements would you recommend for this process?

6. What are the challenges specifically faced by students identified with autism in transitioning to college?

7. What strengths do students with autism bring to this transition to college?

8. Given a "blue sky" environment, what would you request to improve the transition experience for students with autism to college?

9. What else would you like to share regarding your experiences with students with autism and their transition to college?

10. Research indicates that many students identified with autism indicate that they intend to seek a postsecondary education yet do not persist in that endeavor. What might be the causes of such desistence?

Thank you again for your time and candor. I will be contacting you if a follow-up interview is needed.

Future Interview Topics/Questions:

- Continued experiences

- Successes

- Challenges

- Changes over time 
Interview Guide - California community college stakeholder

Thank you for agreeing to be part of my study on views and experiences relating to transition and success for students identified with autism. Over the course of about 30-45 minutes I will be asking you a few questions. You are free to skip a question or not answer something at any point if it might be uncomfortable for you. Think of this as us having a conversation. Depending on your answers, I may ask additional questions to further explore your views.

1. What is your role in transitioning students with autism to college?

2. What is the process used by this community college to facilitate the successful transition of students identified with autism to college?

3. What stakeholders are involved in this process? (title not name)

4. What are the strengths of this process?

5. What improvements would you recommend for this process?

6. What are the challenges specifically faced by students identified with autism in transitioning to college?

7. What strengths do students with autism bring to this transition to college?

8. Given a "blue sky" environment, what would you request to improve the transition experience for students with autism to college?

9. What else would you like to share regarding your experiences with students with autism and their transition to college?

Thank you again for your time and candor. I will be contacting you if a follow-up interview is needed.

Future Interview Topics/Questions:

- Continued experiences

- Successes

- Challenges

- Changes over time 


\section{Appendix G \\ Campus Tour/Interview}

Interview Guide \#4 - Student "Shadow” Interview

Thank you for agreeing to this interview as part of my study on views and experiences with transition from high school and success at community college. Over the course of this interview I will be asking you a few questions. You are free to skip a question or not answer something at any point if it might be uncomfortable for you. Think of this as us having a conversation. Depending on your answers, I may ask additional questions to further explore your views.

1. On a typical day How do you get to school?

2. On a typical day, where do you go on campus?

a. What buildings/places do you go to?

b. Where do you like to "hang out"?

3. Tell me about a place on campus where you feel comfortable.

a. What is it about this place that feels comfortable?

4. Tell me about a place on campus where you feel less comfortable.

a. What is it about that place that feels less comfortable?

5. Tell me about the classes you are taking.

a. What is your favorite class... Why is it your favorite?

b. Which class is your least favorite... Why is it your least favorite?

c. Are there classes where you don't feel comfortable? Do you know why you don't feel comfortable?

6. Where at school do you feel most supported? Why?

7. Where at school do you feel less support? Why?

8. Where is the Disability Services office?

a. What has it done that has been good for you?

b. What else could it do?

c. What could it do better? Why is this important?

9. Do you stay after school or leave right away...Why?

10. So, how do you feel about Community college so far? ... Tell me more...

11. Is Community college what you thought it would be like? ...Tell me more...

12. How has it lived up to what you thought it would be like? ...Tell me more...

13. How has it not lived up to what you thought it would be like? ...Tell me more...

14. What else would you like to share about the school?

Thank you for participating in my study. 


\section{Appendix $\mathbf{H}$ \\ School Profile}

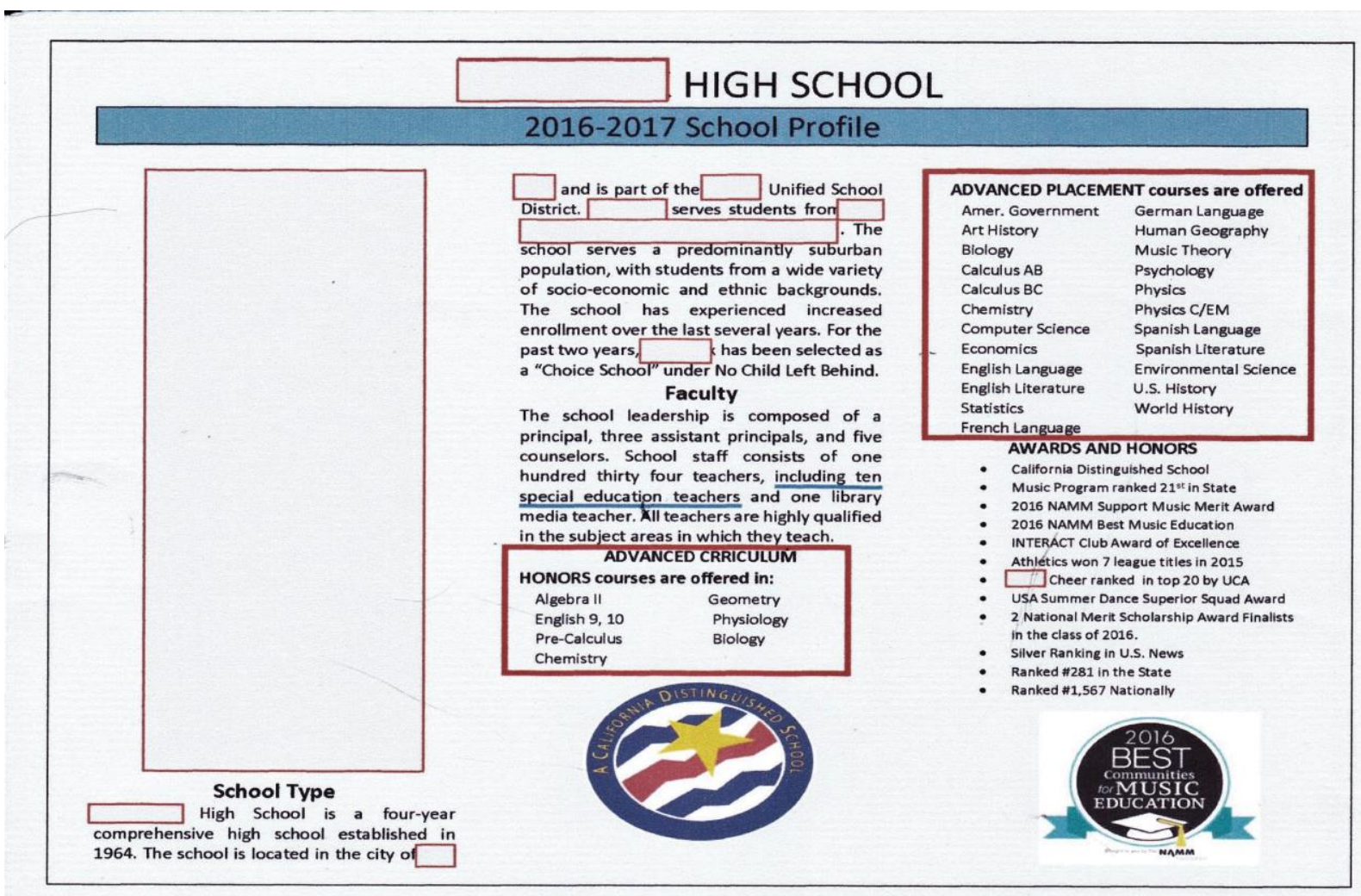

\begin{tabular}{|c|c|c|}
\hline \multicolumn{3}{|c|}{$\begin{array}{l}2016 \text { ADVANCED PLACEMENT } \\
\text { SCORES }\end{array}$} \\
\hline AP Test Subject & $\begin{array}{c}\text { Number } \\
\text { Tested }\end{array}$ & $\begin{array}{c}\text { Average } \\
\text { Score }\end{array}$ \\
\hline Art History & 20 & 2.55 \\
\hline Biology & 71 & 3.24 \\
\hline Calculus AB & 68 & 3.67 \\
\hline Calculus BC & 43 & 4.23 \\
\hline Chemistry & 68 & 3.02 \\
\hline Chinese Language & 2 & 4.00 \\
\hline Computer Science A & 1 & 4.00 \\
\hline English Language & 214 & 2.89 \\
\hline English Literature & 143 & 2.80 \\
\hline Environmental Science & 39 & 2.31 \\
\hline French Language & 6 & 2.00 \\
\hline Human Geography & 1 & 5.00 \\
\hline Italian Language & 2 & 5.00 \\
\hline Macroeconomics & 94 & 3.17 \\
\hline Music Theory & 2 & 1.00 \\
\hline Physics 1 & 88 & 1.78 \\
\hline Physics C & 41 & 3.00 \\
\hline Psychology & 111 & 2.72 \\
\hline Spanish Language & 66 & 3.19 \\
\hline Statistics & 28 & 3.11 \\
\hline U.S. History & 133 & 3.14 \\
\hline U.S. Gov't \& Politics & 80 & 3.20 \\
\hline World History & 123 & 2.90 \\
\hline \multicolumn{3}{|c|}{ Percentage of Students Passing AP Exams } \\
\hline 2016 & & \\
\hline 2015 & & \\
\hline
\end{tabular}

\begin{tabular}{|c|c|c|}
\hline \multicolumn{3}{|c|}{ COLLEGE AND ADMISSIONS TEST SCORES } \\
\hline Test & 2016 & 2015 \\
\hline SAT I Critical Reading & 518 & 515 \\
\hline SATIMath & 532 & 525 \\
\hline SATI Writing & 505 & 509 \\
\hline $\begin{array}{l}\text { Universities of Re } \\
\text { UC: Berkeley, Davis, Irv } \\
\text { Merced, Riverside, San } \\
\text { Santa Cruz } \\
\text { Cal Poly: Pomona, San } \\
\text { Cal State: Chico, Domir } \\
\text { Fullerton, Humboldt, L } \\
\text { Sacramento, San Diego, } \\
\text { Francisco, San Jose, Son } \\
\text { Private or Out of State: } \\
\text { State, Azusa Pacific, Bio } \\
\text { Concordia, Denver, Don } \\
\text { Washington, Grand Can } \\
\text { Hollins, Illinois, Johnson } \\
\text { La Sierra, Maryland, MI } \\
\text { Michigan, Michigan Sta } \\
\text { Oklahoma State, Orego } \\
\text { Pomona, Purdue, Stanf } \\
\text { Chattanooga, Towson, } \\
\text { Reno, USC, UTI, Vangua } \\
\text { Washington State, Wes }\end{array}$ & $\begin{array}{l}\text { nt Gra } \\
\text { Los An, } \\
\text { oo, Sant } \\
\text { Obispo } \\
\text { z Hills, } \\
\text { ngeles, } \\
\text { Marco } \\
\text { zana, A } \\
\text { zyU, Chi } \\
\text { can, Du } \\
\text { Hawai } \\
\text { W Wales } \\
\text { liami of } \\
\text { Jorther } \\
\text { Oregon } \\
\text { Tennes } \\
\text { ty, UNL } \\
\text { Washin } \\
\text { nt, Yale }\end{array}$ & $\begin{array}{l}\text { ites } \\
\text { bara, } \\
\text { 3ay, } \\
\text { sas, } \\
\text { ding, } \\
\text { ona, } \\
\text { a }\end{array}$ \\
\hline
\end{tabular}

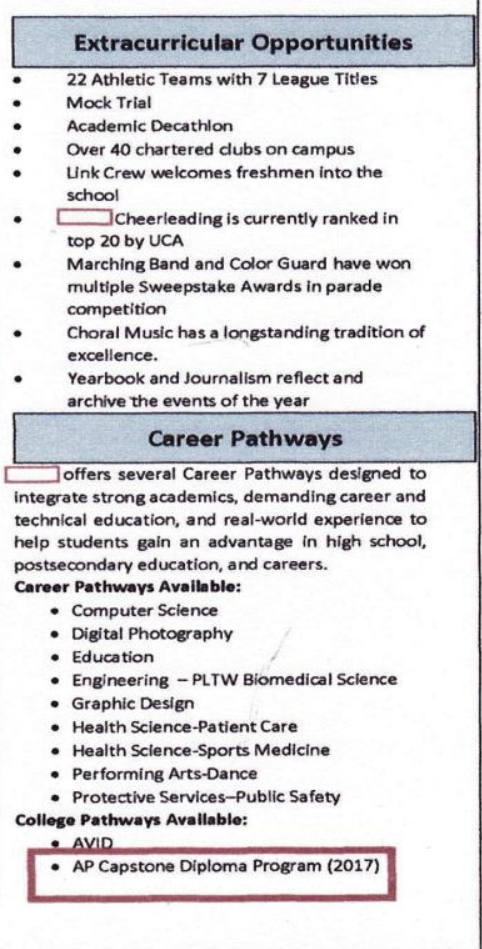




\section{Appendix I}

\section{School Magazine Senior Destinations}

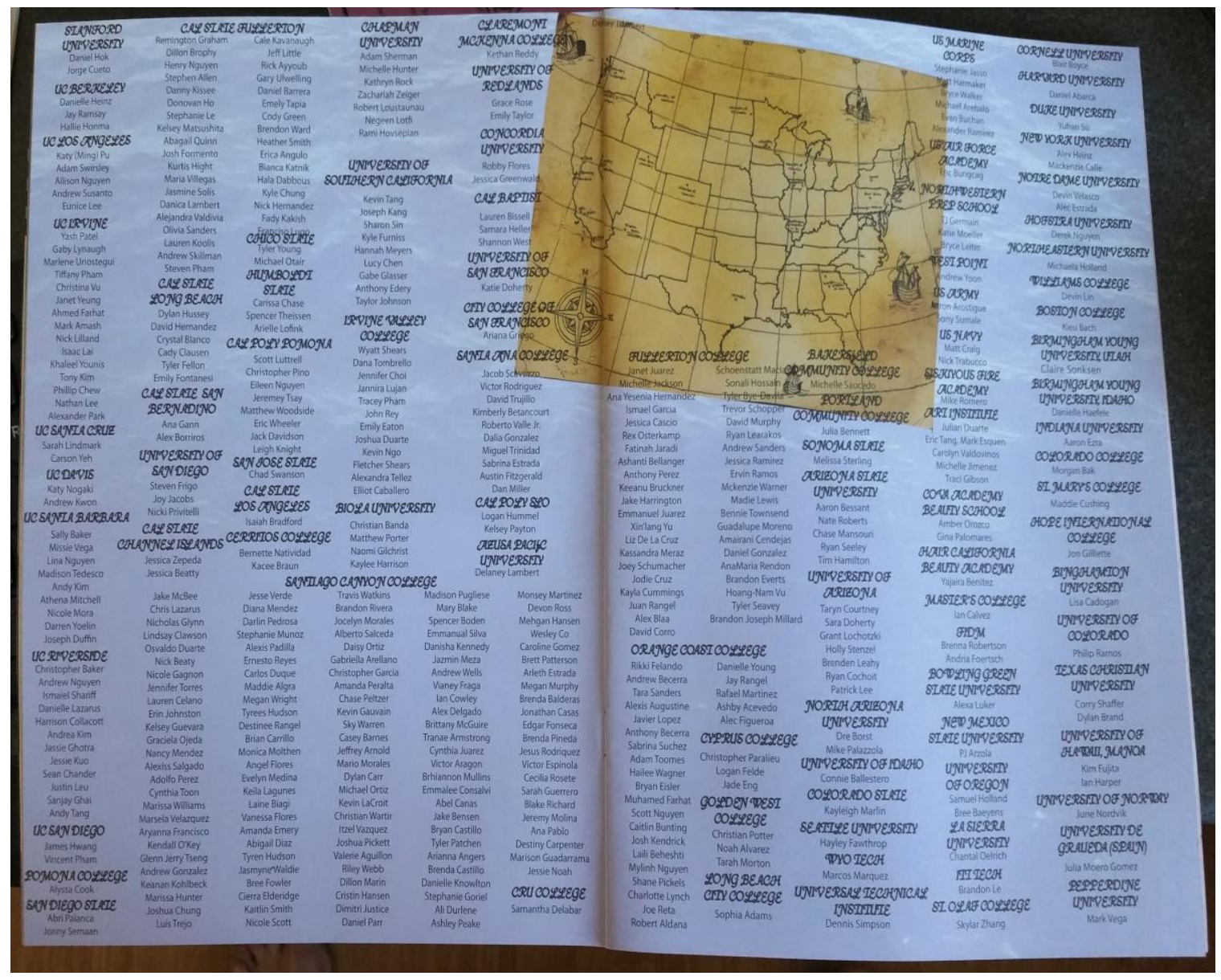




\section{Appendix J}

\section{Sample Individual Transition Plans (ITP)}

\begin{tabular}{|c|c|c|}
\hline \multicolumn{3}{|c|}{ INDIVIDUAL TRANSITION PLANNING (ITP) } \\
\hline \multicolumn{2}{|c|}{ Date of Birth Bt Inv } & IEP Date: $\underline{10 / 16 / 2018}$ \\
\hline Student Invited: $\square Y_{e s} \square$ No & \multicolumn{2}{|c|}{$\begin{array}{l}\text { If Appropriate, and agreed upon, agencies invited: } \\
\square \mathrm{Yes} \square \mathrm{No} \square \mathrm{N} / \mathrm{A}\end{array}$} \\
\hline Describe how the student participated in the process: & $\begin{array}{l}\text { УPresent At Meeting } \\
\square \text { Interest Inventories }\end{array}$ & $\begin{array}{l}\text { УInterview Prior } \\
\square \text { Questionnaire }\end{array}$ \\
\hline \multicolumn{3}{|c|}{ Age-appropriate transition assessments/instruments were used: $\square$ Yes $\square$ No } \\
\hline \multicolumn{3}{|c|}{$\begin{array}{l}\text { Describe the results of the assessments: } \\
\text { Joshua and case manager had discussions about Joshua's future plans and interests. }\end{array}$} \\
\hline \multicolumn{3}{|c|}{ Student's Post Secondary Goal Training or Education (Required): } \\
\hline Upon completion of school I will look into going to a Jr. College. & \multicolumn{2}{|c|}{$\begin{array}{l}\text { Transition Service Code as Appropriate: } \\
890 \text { Other Transition Service } \\
\text { Activities to Support Post Secondary Goal: } \\
\quad \text { will be encouraged to attend on campus visits from Jr. } \\
\text { Colleges, and has access to the computer lab where he can } \\
\text { talk to the career center personnel and/or look up college and } \\
\text { job information on the computers. } \\
\text { Community Experiences as Appropriate: } \\
\text { In addition, will be encouraged to attend college fairs on } \\
\text { campus, and will be encouraged to visit the career center in the } \\
\text { VPHS library Monday through Thursday. }\end{array}$} \\
\hline $\begin{array}{l}\text { Linked to Annual Goal \# All } \\
\text { Person/Agency Responsible: Education Specialist }\end{array}$ & \multicolumn{2}{|c|}{$\begin{array}{l}\text { Related Services as Appropriate: } \\
330 \text { Specialized Academic/nstruction }\end{array}$} \\
\hline
\end{tabular}

Student's Post Secondary Goal Employment (Required):

\begin{tabular}{|l|l|}
\hline $\begin{array}{l}\text { Upon completion of school I will look for part time or full time } \\
\text { work in something I'm interested in. }\end{array}$ & $\begin{array}{l}\text { Transition Service Code as Appropriate: } \\
890 \text { Other Transition Service }\end{array}$ \\
\hline $\begin{array}{ll}\text { Activities to Support Post Secondary Goal: } \\
\text { Once is } 16 \text { he can apply for a work permit through the } \\
\text { career center in the library. In addition, Joshua will have access } \\
\text { to local jobs that are available to students at VPHS, through the } \\
\text { career center and workability. } \\
\text { Community Experiences as Appropriate: } \\
\text { will be encouraged to look in the community for job }\end{array}$ \\
$\begin{array}{ll}\text { wanted signs or even online for job postings in the community. } \\
\text { Related Services as Appropriate: } \\
\text { Linked to Annual Goal \# } \underline{\text { All }}\end{array}$ \\
Person/Agency Responsible: Education Specialist
\end{tabular}

Student's Post Secondary Goal Independent Living (As appropriate):

\begin{tabular}{|l|l|}
\hline Upon completion of school I will & Transition Service Code as Appropriate: \\
& $\begin{array}{l}\text { Activities to Support Post Secondary Goal: } \\
\text { Community Experiences as Appropriate: }\end{array}$ \\
\hline Linked to Annual Goal \# & Related Services as Appropriate: \\
\hline Person/Agency Responsible: & \\
\hline
\end{tabular}




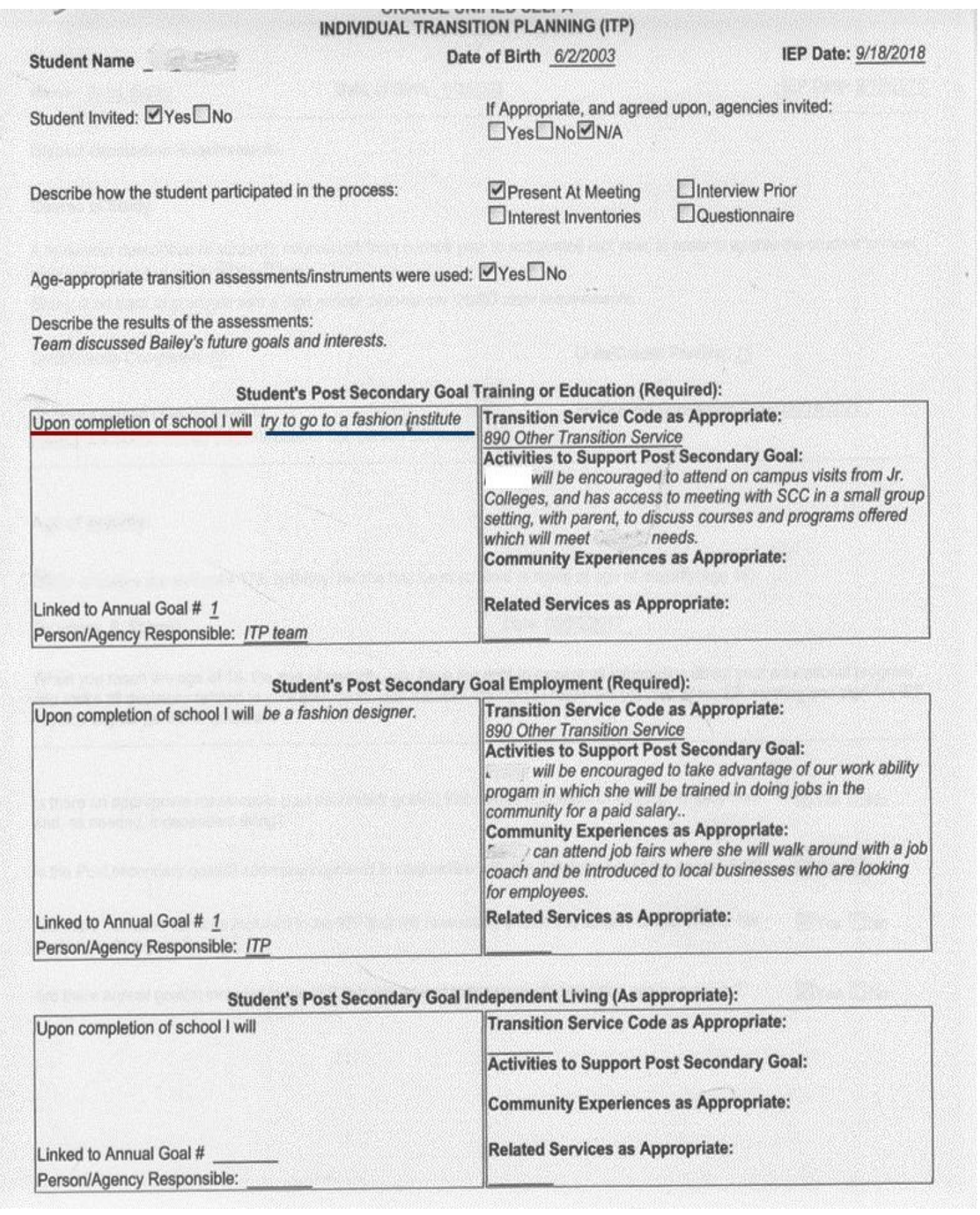




\section{Appendix K}

\section{College Student Equity}

Executive Summary

2017-2019

College recognizes the importance of student equity. The college is committed to ensuring equity tenets are paramount to the development and integration of all campus-wide plans, procedures, and policies. The college established a Student Equity Committee and was part of the participatory governance structure. In 2015, this Student Equity Committee and the Student Success Committee merged to provide a more seamless and collaborative effort between the Student Equity and Student Success and Support Program, creating the Student Success and Equity Committee (SS\&EC). With support from the SS\&EC, campus researchers identified disproportionate impact within the statemandated target populations as validated with data collected and analyzed by our Office of Institutional Effectiveness. This data was then used in the development of strategies that will address the gaps found under the five success indicators. Student Equity plan will remain a living document that directs us annually to provide students with specialized services to guide them toward their academic goals.

\section{Target Populations}

College aligned its research on disproportionate impact with those students identified in the student equity plan template. Using both the " $80 \%$ Rule" and the "percentage gap index as the base metric, multiple groups were found to achieve success at significantly lower rates than those who are in the highest performing groups. As a result, goals and activities were created to address the gaps for the following student groups:

\section{STUDENT GROUPS IDENTIFIED}

Students who identify as: African American, Asian, Filipino, Pacific Islander, Latino/a, White, Native American/Alaskan, Male, Foster Youth, Veterans, Low-Income.

Students who marked "Other/Decline to state", or "More than one race" on their CC Application.

Students who have a documented disability.

It should be noted that while disproportionate impact could not be measured for specific student groups (i.e. Students who are African American, Native American/Alaskan, foster youth, and veterans), because their numbers are too small under a few success indicators, acknowledges these students' place within the state legislation, and will continue to monitor and provide services to ensure their success in all areas identified. 


\section{References}

Adreon, D. (2004). Ten skills to teach your child in preparation for life after high school. Florida Asperger Syndrome Times. Coral Gables, FL: University of Miami Center for Autism and Related Disabilities.

Adreon, D. (2016). Life after high school, ten skills to teach your child. Autism Support Network. Retrieved from http://www.autismsupportnetwork.com/news/life-after -high-school-ten-skills-teach-your-child-autism-223421

Adreon, D., \& Durocher, J. S. (2007). Evaluating the college transition needs of individuals with high-functioning autism spectrum disorders. Intervention in School \& Clinic, 42(5). 271-279. doi:10.1177/10534512070420050201

Aljohani, O. (2016). A comprehensive review of the major studies and theoretical models of student retention in higher education. Higher Education Studies, 6(2), 1-18. doi:10.5539/hes.v6n2p1

American Psychiatric Association (1994). Diagnostic and statistical manual of mental disorders (4th ed.), Washington, DC: American Psychiatric Association.

American Psychiatric Association (2013). Diagnostic and statistical manual of mental disorders. (5th ed.), Washington, DC: American Psychiatric Association. Retrieved from http://www.terapiacognitiva.eu/dwl/dsm5/DSM-5.pdf American Psychiatric Association. (2015). Brief report: The impact of changing from DSM-IV 'Asperger's' to DSM-5 'Autistic Spectrum Disorder' diagnostic labels on stigma and treatment attitudes. Journal of Autism \& Developmental Disorders, 45(10), 3384-3389. doi:10.1007/s10803-015-2485-7 
Anderson, M., Goodman, J., \& Schlossberg, N. (2011). Counseling adults in transition: Linking Schlossberg's theory with practice in a diverse world. (4th ed.). New York, NY: Springer.

Areheart, B.A. (2008). When disability isn't “just right”': The entrenchment of the medical model of disability and the Goldilocks dilemma. Indiana Law Journal, 83(1), 181-232. Retrieved from https://www.repository.law.indiana.edu/cgi /viewcontent.cgi $?$ article $=1193 \&$ context $=\mathrm{ilj}$

Arnett, J. J. (2004). Emerging Adulthood: The Winding Road from the Late Teens Through the Twenties. New York: Oxford University Press.

Astin, A. (1985). Achieving Educational Excellence. A Critical Assessment of Priorities and Practices in Higher Education. San Francisco: Jossey-Bass.

Austin, K. S., \& Peña, E. V. (2017). Exceptional Faculty Members Who Responsively Teach Students with Autism Spectrum Disorders. Journal of Postsecondary Education and Disability, 30(1), 17-32. Retrieved from http://libproxy.chapman.edu/login?url=http://search.ebscohost.com/login.aspx ?direct=true $\&$ AuthType=ip,uid $\& d b=$ eric $\& A N=E J 1144609 \&$ site=eds-live

Barnhart, G. S. (2016). Clinician perspectives of adult high-functioning autism support groups' use of neurodiversity concept (Doctoral Dissertation), Retrieved from ProQuest Dissertations \& Theses database. (No. 100257370)

Bean, J. P., \& Metzner, B.S. (1985). A conceptual model of nontraditional undergraduate student attrition. Review of Educational Research, 55(4), 485-540. doi:10.3102/00346543055004485 
Ben Itzchak, E., \& Zachor, D. A. (2011). Who benefits from early intervention in autism spectrum disorders? Research in Autism Spectrum Disorders, 5(1), 345-350. doi:10.1016/j.rasd.2010.04.018

Birks, M., \& Mills, J. (2011). Grounded theory: A practical guide. London: Sage. Bloch, D., \& Richmond, L. (1998). Soul work: Finding the work you love, loving the work you have. Palo Alto, CA: Davies-Black.

Blume, H. (1998, September). Neurodiversity: On the neurological underpinnings of geekdom. The Atlantic. 142(9). Retrieved from https://www.theatlantic.com/magazine/archive/1998/09/neurodiversity/305909/ Bridges, W. (2004). Transitions: Making sense of life's changes. Cambridge, MA: Da Capo Press.

Bright, J. H., \& Pryor, R. L. (2008). Shiftwork: A chaos theory of careers agenda for change in career counseling. Australian Journal of Career Development, 17(3), 63-72. doi:10.1177/103841620801700309

Brown, K. R. (2012) Institutional practices that support students with autism spectrum disorders in a postsecondary educational setting (Doctoral dissertation). Retrieved from ProQuest Dissertations \& Theses database. (No. 3510801).

Brown, K. R., \& Coomes, M. D. (2016). A spectrum of support: current and best practices for students with Autism Spectrum Disorder (ASD) at community colleges. Community College Journal of Research and Practice, 40(6), 465-479. doi:10.1080/10668926.2015.1067171 
Brown, L. X. (2011, August 9). The Significance of Semantics: Person-First Language: Why It Matters [Web log post]. Retrieved from https://www.autistichoya.com /2011/08/significance-of-semantics-person-first.html

Browning, J., Osborne, L. A., \& Reed, P. (2009). A qualitative comparison of perceived stress and coping in adolescents with and without autistic spectrum disorders as they approach leaving school. British Journal of Special Education, (1), 36-43. doi:10.1111/j.1467-8578.2008.00400.x

Bureau of Labor Statistics. (2017, May 22). 69.7 percent of 2016 high school graduates enrolled in college in October 2016. In TED: The Economics Daily. Retrieved from https://www.bls.gov/opub/ted/2017/69-point-7-percent-of-2016-high -school-graduates-enrolled-in-college-in-october-2016.htm

Bussolari, C. J. \& Goodell, J. A. (2009). Chaos theory as a model for life transitions counseling: Nonlinear dynamics and life's changes. Journal of Counseling and Development, 87, 98-107. doi:1556-6678.2009.tb00555.x

Campbell, J. and Oliver, M. (1996). Disability Politics: Understanding Our Past, Changing Our Future. New York: Routledge.

Cardinal, D., \& Fraumeni-McBride, J. (2017). Increase in rate of autism likely a factor of definition: A multi-state analysis of autism prevalence in U.S. schools, initial report. In Thompson Policy Institute 2017 Summary of Findings. Retrieved from https://www.chapman.edu/education/centers-and-partnerships/thompson-policy -institute/_files/2017-summary-report-final.pdf 
Carroll, J. B. (1963). Model of school learning. Teachers College Record (1900), 64, 723-733. Retrieved from http://libproxy.chapman.edu/login?url=http:// search.ebscohost.com/login.aspx ?direct=true\&AuthType=ip, uid \&db=eir\&AN=520210951\&site=eds-live

Castleman, B. L., \& Page, L. C. (2015). Summer nudging: Can personalized text messages and peer mentor outreach increase college going among low-income high school graduates? Journal of Economic Behavior and Organization, 115, 144-160. doi:10.1016/j.jebo.2014.12.008

Causton-Theoharis, J., \& Theoharis, G. (2009). Creating Inclusive Schools for All Students. Education Digest, (6), 43. Retrieved from http://eds.a.ebscohost.com .libproxy.chapman.edu/eds/pdfviewer/pdfviewer?vid=7\&sid=ecd12301-2a8144f3-bd70-cab436eb53cd\%40sessionmgr4006

Center for Community College Student Engagement (2014). Contingent commitments: Bringing part-time faculty into focus (A special report from the Center for Community College Student Engagement). Austin, TX: The University of Texas at Austin, Program in Higher Education Leadership.

Centers for Disease Control and Prevention. (2018, April 27). Prevalence of Autism Spectrum Disorder among children aged 8 years — Autism and developmental disabilities monitoring network, 11 sites, United States, 2014. In Morbidity and Mortality Weekly Report (MMWR) Retrieved May 4, 2018, from https://www.cdc.gov/mmwr/volumes/67/ss/ss6706a1.htm

Charmaz, K. 2014). Constructing grounded theory: a practical guide through qualitative analysis. Thousand Oaks, CA: Sage. 
Chiang, H., Cheung, Y. K., Hickson, L., Xiang, R., \& Tsai, L. Y. (2012, May 1).

Predictive factors of participation in postsecondary education for high school leavers with autism. Journal of Autism \& Developmental Disorders, 42(5), 685696. doi:10.1007/10803-011-1297-7

Christensen, D. L. (2016, April 1). Prevalence and Characteristics of Autism Spectrum Disorder Among Children Aged 8 Years - Autism and Developmental Disabilities Monitoring Network, 11 Sites, United States, 2012. In Centers for Disease Control and Prevention. Retrieved from https://www.cdc.gov/mmwr /volumes/65/ss/ss6503a1.htm

Cochran, K. L. (2009). Peer mentor program for high school students on the autism spectrum; aimed to decrease reported stress regarding college transition (master's thesis). Retrieved from ProQuest Dissertations \& Theses database. (No. 10157126)

The College Board. Coming to Our Senses: Education and the American Future, 2008. Retrieved from https://secure-media.collegeboard.org/digitalServices/pdf/ advocacy/admissions21century/coming-to-our-senses-college-board-2008.pdf

Creswell, J. W. (2003). Research design: Qualitative, quantitative, and mixed methods approaches. Thousand Oaks, CA: Sage.

Dalrymple, N., \& Indiana Univ., B. I. R. C. for A. (1989). Learning to be Independent and Responsible. Functional Programming for People with Autism: A Series. Retrieved from http://libproxy.chapman.edu/login? url=http://search.ebscohost.com/login.aspx?direct=true\&AuthType=ip, uid $\& d b=$ eric $\& A N=E D 363063 \&$ site $=$ eds-live 
Daly-Cano, M., Vaccaro, A., \& Newman, B. (2015). College student narratives about learning and using self-advocacy skills. Journal of Postsecondary Education and Disability, 28(2), 213-227. Retrieved from http://libproxy.chapman.edu/login ?url=http://search.ebscohost.com/login.aspx?direct=true\&AuthType=ip,uid\&db=e ric $\& \mathrm{AN}=\mathrm{EJ} 1074673 \&$ site $=$ eds-live

DeLeo, C. B. S. (2017). Transitioning students with autism spectrum disorder from school to society (Doctoral dissertation) Retrieved from ProQuest Dissertations \& Theses database (No. 10259820).

Dillon, M. R. (2007). Creating supports for college students with Asperger Syndrome through collaboration. College Student Journal, 41(2), 499-504. doi:10.1177/1088357614523121

Dipeolu, A., Storlie, C., \& Johnson, C. (2014). Transition to college and students with high functioning autism spectrum disorder: Strategy considerations for school counselors. Journal of School Counseling, 12(11). Retrieved from http://libproxy.chapman.edu/login?url=http://search.ebscohost.com/login.aspx? direct=true $\&$ AuthType $=$ ip, $u i d \& d b=$ ric $\& A N=E J 1034736 \&$ site=eds-live

Drouin, M., Reining, L., Flanagan, M., Carpenter, M., \& Toscos, T. (2018). College Students in Distress: Can Social Media Be a Source of Social Support? College Student Journal, 52(4), 494-504. Retrieved from http://libproxy.chapman.edu/login?url=http://search.ebscohost.com/login.aspx? direct=true $\&$ AuthType $=i p, u i d \& d b=s 3 h \& A N=134341758 \&$ site $=$ eds-live

Durkheim, E. (1952). Suicide: A study in sociology (J. Spaulding \& G. Simpson, trans). London: Routledge \& Kegan Paul Ltd. 
Ebel, K. L. (2015). Transition from high school to postsecondary education: Narratives of parents of college students with autism spectrum disorders (Doctoral dissertation). Retrieved from ProQuest Dissertations \& Theses database. (No. 3711218)

Eckhardt, S. C. (2017). Social Media Use as a Third Place for Community College Students with Autism Spectrum Disorder. (Doctoral dissertation) Retrieved from ProQuest Dissertations \& Theses database. (No. 10690523)

Elias, R., Muskett, A. E., \& White, S. W. (2019). Educator Perspectives on the Postsecondary Transition Difficulties of Students with Autism. Autism: The International Journal of Research and Practice, 23(1), 260-264. doi:10.1177/1362361317726246

Evans, N. J., Forney, D. S., Guido, F. M., Patton, L. D., \& Renn, K. A. (2010). Student development in college: Theory, research, and practice (2nd ed.). San Francisco, CA: Jossey-Bass.

Ferguson, H., Myles, B., and Hagiwara, T. (2005). Using a personal digital assistant to enhance the independence of an adolescent with Asperger Syndrome. Education and Training in Developmental Disabilities, 40(1), 60. Retrieved from http://libproxy.chapman.edu/login?url=http://search.ebscohost.com/login.aspx ?direct=true \&AuthType=ip,uid\&db=edsjsr\&AN=edsjsr.23879772\&site=eds-live

Fox, C. (2011). Expectations and the post transition of young adults with an autism spectrum disorder to postsecondary education (Doctoral dissertation). Retrieved from ProQuest Dissertations and Theses database. (No. 3452989) 
Gallagher, S. (2004). Understanding interpersonal problems in autism: Interaction theory as an alternative to theory of mind. Philosophy, Psychiatry, \& Psychology, (3), 199. Retrieved from https://doi-org.libproxy.chapman.edu/10.1353/ppp.2004 .0063

Gelbar, N., Smith, I., \& Reichow, B. (2014). Systematic review of articles describing experience and supports of individuals with autism enrolled in college and university programs. Journal of Autism \& Developmental Disorders, 44(10), 2593-2601. doi:10.1007/s10803-014-2135-5

Giarelli, E., Ruttenberg, J., \& Segal, A. (2013). Bridges and barriers to successful transitioning as perceived by adolescents and young adults with Asperger syndrome. Journal of Pediatric Nursing, 28(6), 563-574. doi:10.1016/j.pedn.2012.12.010

Gibbons, M. M., Cihak, D. F., Mynatt, B., \& Wilhoit, B. E. (2015). Faculty and Student Attitudes toward Postsecondary Education for Students with Intellectual Disabilities and Autism. Journal of Postsecondary Education and Disability, 28(2), 149-162. Retrieved from http://libproxy.chapman.edu /login?url=http://search.ebscohost.com/login.aspx?direct=true\&AuthType=ip ,uid\&db=eric\&AN=EJ1074661\&site=eds-live

Gillot, A., \& Standen, P. J. (2007). Levels of anxiety and sources of stress in adults with autism. Journal of Intellectual Disabilities, 11(4), 359-370. doi:10.1177/1744629507083585

Glaser, B. G., \& Strauss, A. L. (1967) The discovery of grounded theory; strategies for qualitative research. Chicago, Aldine Pub. Co. 
Glennon, T. J. (2001). The stress of the university experience for students with Asperger syndrome. Work, 17(3), 183-190. Retrieved from http://libproxy.chapman.edu /login?url=http://search.ebscohost.com/login.aspx?direct=true\&AuthType=ip ,uid\&db=rzh\&AN=106920477\&site=eds-live

Gobbo, K., \& Shmulsky, S. (2012). Classroom Needs of community college students with Asperger's disorder and autism spectrum disorders. Community College Journal of Research and Practice, 36(1), 40-46.

doi:10.1080/10668920903381813

Goodley, D. (2001). Learning difficulties, the social model of disability and impairment: Challenging epistemologies. Disability \& Society, 16, 207-231. doi:10.1080/09687590120035816

Goyette, K. A. (2008). College for some to college for all: Social background, occupational expectations, and educational expectations over time. Social Science Research, 37(2), 461-484. doi:10.1016/j.ssresearch.2008.02.002

Hadjikakou, K., \& Mnasonos, M. (2012). Investigating the attitudes of head teachers of Cypriot primary schools towards inclusion. Journal of Research in Special Educational Needs, 12(2), 66-81. doi:10.1111/j.1471-3802.2010.01195.x

Helps, S., Newsom-Davis, I., \& Callias, M. (1999). Autism: The teacher's view. Autism: The International Journal of Research \& Practice, 3(3), 287-298. doi:10.1177/1362361399003003006 
Highlen, D. (2017). Helping students with autism spectrum disorder at the community college: What does the research say? What can you do? Community College Journal of Research and Practice, 41(7), 447-454.

doi; $10.1080 / 10668926.2016 .1199334$

Hill, L. D. (2008). School Strategies and the "College-Linking” Process: Reconsidering the Effects of High Schools on College Enrollment. Sociology of Education, (1), 53. Retrieved from http://libproxy.chapman.edu/login? url=http://search.ebscohost.com/login.aspx?direct=true\&AuthType=ip ,uid\&db=edsbl\&AN=RN224987478\&site=eds-live

Hillary Clinton Acceptance Speech. (2016, July 28). In C-SPAN. Retrieved from https://www.c-span.org/video/?412848-101/hillary-clinton-acceptance-speech

Hodgson, A. R., Freeston, M. H., Honey, E., \& Rodgers, J. (2017). Facing the Unknown: Intolerance of Uncertainty in Children with Autism Spectrum Disorder. Journal of Applied Research in Intellectual Disabilities, (2), 336. doi:10.1111/jar.12245

Holland, M. M. (2015). College for All and Community College for None: Stigma in High-Achieving High Schools. Teachers College Record, 117(5). Retrieved from http://libproxy.chapman.edu/login?url=http://search.ebscohost.com/login.aspx ?direct=true $\&$ AuthType $=$ ip, uid $\& d b=e d s w s s \& A N=000356234600005 \&$ site $=$ eds -live 
Howard, A. L., Strickland, N. J., Murray, D. W., Tamm, L., Swanson, J. M., Hinshaw, S. P., ... Molina, B. S. G. (2016). Progression of impairment in adolescents with attention-deficit/hyperactivity disorder through the transition out of high school:

Contributions of parent involvement and college attendance. Journal of Abnormal Psychology, 125(2), 233-247. doi:10.1037/abn0000100

Hudson, F. M. (1991). The adult years: Mastering the art of self-renewal. San Francisco, CA: Jossey Bass.

Hudson, F. M. (1999). The adult years: Mastering the art of self-renewal (Rev. ed.). San Francisco, CA: Jossey Bass.

Humes, E. (2006). Over Here: How the G.I. Bill Transformed the American Dream. New York, NY: Diversion Books.

Individuals with Disabilities Education Act. (1990). PL 101-476 U.S.C. Retrieved from https://www.congress.gov/bill/101st-congress/senate-bill/1824/text

Institute of Education Sciences. (2018, March 1). Fast Facts. In National Center for Education Statistics. Retrieved from https://nces.ed.gov/fastfacts/display .asp?id=372

Janiga, S. J., \&. Costenbader, V. (2002). The transition from high school to postsecondary education for students with learning disabilities: A survey of college service coordinators. Journal of Learning Disabilities, 35(5), 463-470. doi:10.1177/00222194020350050601

Jones, J. A. (2016). The social experiences of young adults with autism as they transition from high school to college. (Doctoral dissertation). Retrieved from ProQuest Dissertations and Theses database. (No. 10141671) 
Kelley, L., \& Joseph, B. (n.d.). Rethinking higher education for students with autism spectrum disorders: The importance of adult transitions. Unpublished manuscript, University of San Diego, San Diego, CA. Retrieved from https://www.sandiego.edu/disability/documents/Rethinking_Higher_Education _for_Students_with_Autism_Spectrum_Disorders_APA.pdf

Kimball, E. W., Moore, A., Vaccaro, A., Troiano, P. F., \& Newman, B. M. (2016). College students with disabilities redefine activism: Self-advocacy, storytelling, and collective action. Journal of Diversity in Higher Education, 9(3), 245-260. doi:10.1037/dhe0000031

Kurth, N., \& Mellard, D. (2006). Student Perceptions of the Accommodation Process in Postsecondary Education. Journal of Postsecondary Education and Disability, 19(1), 71-84. Retrieved from http://libproxy.chapman.edu /login?url=http://search.ebscohost.com/login.aspx?direct=true\&AuthType=ip,uid $\& \mathrm{db}=$ eric $\& \mathrm{AN}=\mathrm{EJ} 844625 \&$ site $=$ eds-live

Klein, A. (2017). Betsy DeVos Sounds Off on Workforce Readiness, Alternatives to College. Education Week, (14). Retrieved from http://libproxy.chapman.edu /login?url=http://search.ebscohost.com/login.aspx?direct=true\&AuthType=ip,uid $\& \mathrm{db}=$ edsgao $\& \mathrm{AN}=$ edsgcl.518304495\&site=eds-live

Klin, A., \& Volkmar, F. R. (n.d.). History of Asperger's disorder. In Psych Central. Retrieved from http://psychcentral.com/lib/history-of-aspergers-disorder/

Koegel, L. K., Ashbaugh, K., Koegel, R. L., Detar, W. J., \& Regester, A. (2013). Increasing socialization in adults with Asperger's syndrome. Psychology in The Schools, 50(9), 899. doi:10.1002/pits.21715 
Kuh, G., Kinzie, J., Cruce, T., Shoup, R., \& Gonyea, R. M. (2007). Connecting the Dots: Multi-Faceted Analyses of the Relationships between Student Engagement Results from the NSSE, and the Institutional Practices and Conditions That Foster Student Success (Lumina Foundation for Education Grant \# 2518). Retrieved from Indiana University Bloomington, Center for Postsecondary Research website: http://nsse.indiana.edu/pdf/Connecting_the_Dots_Report.pdf

Layden, S., Hendricks, D., Inge, K., Sima, A., Erickson, D., Avellone, L., \& Wehman, P. (2018). Providing online professional development for paraprofessionals serving those with ASD: Evaluating a statewide initiative. Journal of Vocational Rehabilitation, 48(2), 285-294. doi:10.3233/JVR-180932

Lazaratou, H., Economou, M., \& Dikeos, D. (2017). The necessity of early intervention in autism. [Letter to the editor]. The Journal of Pediatrics, 184, 240-241. doi:10.1016/j.jpeds.2016.12.030

Lee, C., Mathur, S. R., McCoy, K. M. \& Zucker, S. H. (2014). ASD academic transitions: Trends in parental perspective. Education and Training in Autism and Developmental Disabilities, 49(4), 576-593. Retrieved from https://www-jstororg.libproxy.chapman.edu/stable/24582353?seq=1\#metadata_info_tab_contents

Levinson, E. 1., \& Ohler, D. L. (1998). Transition from high school to college for students with learning disabilities: needs, assessment, and services. High School Journal, 82(1), 62-69. Retrieved from http://libproxy.chapman.edu /login?url=http://search.ebscohost.com/login.aspx?direct=true\&AuthType=ip,uid $\& \mathrm{db}=$ edsbl\&AN=RN057151432\&site=eds-live 
Li, C., Wong, N. K., Sum, R. K. W., \& Yu, C. W. (2019). Preservice Teachers'

Mindfulness and Attitudes Toward Students with Autism Spectrum Disorder: The Role of Basic Psychological Needs Satisfaction. Adapted Physical Activity Quarterly, 36(1), 150. doi:10.1123/apaq.2018-0044

Locke, J., Rotheram-Fuller, E., \& Kasari, C. (2012). Exploring the Social Impact of Being a Typical Peer Model for Included Children with Autism Spectrum Disorder. Journal of Autism \& Developmental Disorders, 42(9), 1895-1905. doi:10.1007/s10803-011-1437-0

MacLeod, A., \& Green, S. (2009). Beyond the books: Case study of a collaborative and holistic support model for university students with Asperger syndrome. Studies in Higher Education, 34(6), 631-646. doi:10.1080/03075070802590643.

Martin, C. S. (2016). Exploring the impact of the design of the physical classroom environment on young children with autism spectrum disorder (ASD). Journal of Research in Special Educational Needs, 16(4), 280-298.

doi:10.1111/1471-3802.12092

Martinez, D. C., Conroy, J. W., \& Cerreto, M. C. (2012). Parent involvement in the transition process of children with intellectual disabilities: The influence of inclusion on parent desires and expectations for postsecondary education. Journal of Policy and Practice in Intellectual Disabilities, 9(4), 279-288.

doi:10.1111/jppi.12000 
Mason, R. A., Rispoli, M., Ganz, J. B., Boles, M. B., \& Orr, K. (2012). Effects of video modeling on communicative social skills of college students with Asperger syndrome. Developmental Neurorehabilitation, 15(6), 425-434. doi:10.3109/17518423.2012.704530

Mathews, T. L., Vatland, C., Lugo, A. M., Koenig, E. A., \& Gilroy, S. P. (2018). Training Peer Models to Promote Social Skills: Considerations for Practice. Focus on Autism and Other Developmental Disabilities, 33(3), 160-170. doi: $10.1177 / 1088357617735814$

Matson, J. L., \& Kozlowski, A. M. (2011). The increasing prevalence of autism spectrum disorders. Research in Autism Spectrum Disorders, 5418-425. doi:10.1016/j.rasd.2010.06.004

McCoy, D. L. (2014). A phenomenological approach to understanding first-generation college students of color transitions to one "extreme" predominantly white institution. College Student Affairs Journal, 32(1), 155-169. Retrieved from http://libproxy.chapman.edu/login?url=http://search.ebscohost.com/login.aspx ?direct=true $\&$ AuthType $=$ ip, uid $\& d b=e f t \& A N=97828437 \&$ site=eds-live

McKeon, B., Alpern, C. S., \& Zager, D. (2013). Promoting academic engagement for college students with autism spectrum disorder. Journal of Postsecondary Education and Disability, 26(4), 353-366. Retrieved from http://libproxy.chapman.edu/login?url=http://search.ebscohost.com/login.aspx ?direct=true \&AuthType=ip,uid\&db=eric \&AN=EJ1026894\&site=eds-live 
Meng, D. (2014). The biomedical model of medicine and traditional Chinese medicine. In University of British Columbia. Retrieved from http://social.eli.ubc.ca /2014/08/27/the-biomedical-model-of-medicine-and-traditional-chinese-medicine/

Minner, S., \& Prater, G. (1984). College Teachers’ Expectations of LD

Students. Intervention in School \& Clinic, 20(2), 225-229.

doi:10.1177/105345128402000213

Mitchell, P., Parsons, S., \& Leonard, A. (2007). Using virtual environments for teaching social understanding to 6 adolescents with autistic spectrum disorders. Journal of Autism and Developmental Disorders, 37(3), 589-600. Retrieved from http://libproxy.chapman.edu/login?url=http://search.ebscohost.com/login.aspx ?direct=true \&AuthType=ip,uid\&db=rzh\&AN=105978962\&site=eds-live

Mitchell, W., \& Beresford, B. (2014). Young people with high-functioning autism and Asperger's syndrome planning for and anticipating the move to college: What supports a positive transition? British Journal of Special Education, 41(2), 151171. doi:10.1111/1467-8578.12064

Morgan, Y., Sinatra, R., \& Eschenauer, R. (n.d.). A Comprehensive Partnership Approach Increasing High School Graduation Rates and College Enrollment of Urban Economically Disadvantaged Youth. Education and Urban Society, 47(5), 596-620. doi:10.1177/0013124514536437

Moore, D., McGrath, P., \& Thorpe, J. (2000). Computer-aided learning for people with autism - A framework for research and development. Innovations in Education and Training International, 37(3), 218-228.

doi:10.1080/13558000050138452 
Morrison, P. S., \& Clark, W. A. V. (2016). Loss aversion and duration of residence. Demographic Research, 35(1), 1079-1100. doi:10.4054/DemRes.2016.35.36

Müller, E., Schuler, A., \& Yates, G. (2008). Social challenges and supports from the perspective of individuals with Asperger syndrome and other autism spectrum disabilities. Autism: The International Journal of Research \& Practice, 12(2), 173-190. doi:10.1177/1362361307086664

Neville, R., \& White, S. (2011). College students' openness toward autism spectrum disorders: Improving peer acceptance. Journal of Autism \& Developmental Disorders, 41(12), 1619-1628. doi:10.1007/s10803-011-1189-x.

Olson, A., Leko, M. M., \& Roberts, C. A. (2016). Providing Students with Severe Disabilities Access to the General Education Curriculum. Research and Practice for Persons with Severe Disabilities, 41(3):143-157. doi:10.1177/1540796916651975

Pascarella, E. (1985). College Environmental Influences on Learning and Cognitive Development: A Critical Review and Synthesis. In Smart, J. (Eds.), Higher Education: Handbook of Theory and Research. New York: Agathon.

Pascarella, E. \& Terenzini, P. (1991). How college affects students. Findings and insights from twenty years of research. San Francisco: Jossey Bass

Peña, E., \& Kocur, J. (2013). Parents' experiences in the transition of students with autism spectrum disorders to community college. Journal of Applied Research in the Community College, 20(2), 29-36. Retrieved from http://libproxy.chapman.edu/login?url=http://search.ebscohost.com/login.aspx ?direct=true $\&$ AuthType $=$ ip ,uid $\& d b=e d o \& A N=97405822 \&$ site=eds-live 
Perner, L. (2002, July). Preparing to be nerdy where nerdy can be cool: College planning for the high functioning student with autism. Paper presented at the $33^{\text {rd }}$ annual conference of the Autism Society of America, Indianapolis, IN. Abstract retrieved from http://www.aspennj.org/pdf/information/articles/college-planning-for-the -high-functioning-student-with-autism.pdf

Perry, M., Khalilnaji-Otto, N., Brackenridge, K., \& Stanford University, Policy Analysis for California Education (PACE). (2018). Summer Learning - A Smart Investment for California School Districts. PACE Continuous Improvement Brief 01 -18. Policy Analysis for California Education, PACE. Retrieved from https://edpolicyinca.org/sites/default/files/summer-learning.pdf

Pew Research Center. (2014, February 11). "The Rising Cost of Not Going to College”. In Pew Research Center. Retrieved from http://www.pewsocialtrends.org /2014/02/11/the-rising-cost-of-not-going-to-college/

Pierson, M. R., \& Howell, E. J. (2013). Two high schools and the road to full inclusion: A comparison study. Improving Schools, 16(3), 223-231. doi: $10.1177 / 1365480213501063$

Poleyeff, L. (2015). A grounded theory investigation of progressing though college with an autism spectrum disorder (Doctoral dissertation). doi:10.7282/T3G162MZ

Prince-Hughes, D. (2002). Aquamarine blue 5: Personal stories of college students with autism. Athens: Swallow Press/Ohio University Press.

Prince-Hughes, D. (2004). Songs of the gorilla nation: My journey through autism. New York, NY: Harmony Books. 
Pugliese, C. E., \& White, S. W. (2013). Brief report: Problem solving therapy in college students with autism spectrum disorders: Feasibility and preliminary efficacy. Journal of Autism and Developmental Disorders. 44(3), 719-729. doi:10.1007/s10803-013-1914-8.

Rendón, L. I. (1994). Validating culturally diverse students: Toward a new model of learning and student development. Innovative Higher Education, 19(1), 1933-51. Retrieved from https://www.csuchico.edu/ourdemocracy/_assets/documents /pedagogy/rendon,-1.-1994---validation-theory.pdf

Rendón, L. I. (2002). Community college Puente: A validating model of education. Educational Policy: An Interdisciplinary Journal of Policy and Practice, 16(4), 642-667. Retrieved from http://libproxy.chapman.edu/login?url=http://search .ebscohost.com/login.aspx $?$ direct=true $\&$ AuthType $=i p, u i d \& d b=m z h \& A N=200287$ $1325 \&$ site $=$ eds-live

Rendón Linares, L. I., \& Muñoz, S. M. (2011). Revisiting Validation Theory: Theoretical Foundations, Applications, and Extensions [Electronic version]. Enrollment Management Journal, 5(2), 12-33. Retrieved from https://www.researchgate.net /publication/282294812_Revisiting_validation_theory_Theoretical_foundations_a pplications_and_extensions

Roberts, K. D. (2010). Topic areas to consider when planning transition from high school to postsecondary education for students with autism spectrum disorders. Focus on Autism and Other Developmental Disabilities, 25(3), 158-162. doi:10.1177/1088357610371476 
Robertson, S. M., \& Ne'eman, A. D. (2008). Autistic acceptance, the college campus, and technology: Growth and neurodiversity in society and academia. Disability Studies Quarterly, 28(4), 14. doi:10.18061/dsq.v28i4.146

Rochford, J. A., O’Neill, A., Gelb, A., Ross, K. J., \& Stark Education Partnership. (2011). Reaching for 80\%: How Post Secondary Opportunities in High Schools Are Changing the College Going Culture in Stark County, Ohio. Stark Education Partnership. Retrieved from http://libproxy.chapman.edu/login ?url=http://search.ebscohost.com/login.aspx?direct=true\&AuthType=ip,uid\&db=e ric $\& A N=E D 525146 \&$ site $=$ eds-live

Roschelle, A. R., \& Kaufman, P. (2004). Fitting in and fighting back: Stigma management strategies among homeless kids. Symbolic Interaction, 27(1), 23-46. doi:10.1525/si.2004.27.1.23

Sanford, C., Newman, L., Wagner, M., Cameto, R., Knokey, A., Shaver, D., \& National Center for Special Education Research (2011). The post-high school outcomes of young adults with disabilities up to 6 years after high school: Key findings from the National Longitudinal Transition Study-2 (NLTS2). NCSER 2011-3004. National Center for Special Education Research. Retrieved from http://libproxy.chapman.edu/login?url=http://search.ebscohost.com/login.aspx ?direct=true $\&$ AuthType=ip,uid $\& \mathrm{db}=$ eric $\& A N=E D 523539 \&$ site=eds-live 
Sayman, D. M. (2015). I still need my security teddy bear: Experiences of an individual with autism spectrum disorder in higher education. Learning Assistance Review, 20(1), 77-98. Retrieved from http://libproxy.chapman.edu/login ?url=http://search.ebscohost.com/login.aspx?direct=true\&AuthType=ip,uid\&db=e ric $\& \mathrm{AN}=\mathrm{EJ} 1058012 \&$ site $=$ eds-live

Schlossberg, N. K. (1981). Model for analyzing human adaptation to transition. Counseling Psychologist, 9(2), 2-18. doi:10.1177/001100008100900202

Schlossberg, N. K. (2011). The challenge of change: The transition model and its applications. Journal of Employment Counseling, 48(4), 159-162. doi:10.1002/j.2161-1920.2011.tb01102.x

Schlossberg, N. K., Waters, E. B., \& Goodman, J. (1995). Counseling adults in transition: Linking practice with theory. New York, NY: Springer.

Shattuck, P. T., Narendorf, S. C., Cooper, B., Sterzing, P. R., Wagner, M., \& Taylor, J. L. (2012). Postsecondary education and employment among youth with an autism spectrum disorder. Pediatrics, 129(6), 1042-1049. doi:10.1542/peds.2011-2864

Shepherd, J. (2015). "Hopefully if I like get the right support at college, I'll be able to like find my way and all that if you know what I mean?" Experiences of transition from special school to mainstream college for young people with autism. (Doctoral dissertation). Retrieved from ProQuest Dissertations and Theses database. (No. 1857774361) 
Shmulsky, S., Gobbo, K., \& Donahue, A. (2015). Groundwork for success: A college transition program for students with ASD. Journal of Postsecondary Education and Disability, 28(2), 235-241. Retrieved from http://libproxy.chapman.edu/login ?url=http://search.ebscohost.com/login.aspx?direct=true\&AuthType=ip,uid\&db=e ric $\& A N=E J 1074676 \&$ site $=$ eds-live

Shore, S. (2003). Helping your child to help him/her self: Beginning self-advocacy. Autism Research Institute. Retrieved from http://www.autism.com/individuals /beginningselfadvocacy.htm

Shyman, E. (2016). The reinforcement of ableism: Normality, the medical model of disability, and humanism in applied behavior analysis and ASD. Intellectual and Developmental Disabilities, 54(5), 366-376,378,380. doi:10.1352/1934-9556 $-54.5 .366$

Spady, W. (1970). Dropouts from higher education: An interdisciplinary review and synthesis. Interchange, 1(1), 64-85. doi:10.1007/BF02214313

Stallings, J. (1980). Allocated academic learning time revisited, or beyond time on task. Educational Researcher, 9, 11-16. doi:10.2307/1175185

Supiano, B. (2016). What's the right public message about going to college? The Chronicle of Higher Education, 63(2), 10. Retrieved from http://libproxy.chapman.edu/login?url=http://search.ebscohost.com/login.aspx ?direct=true $\&$ AuthType=ip,uid $\&$ db=edsggr $\& A N=$ edsgcl. 463632875 $\&$ site=eds-live 
Sutton-Young, T. (2015). Supporting Autism Spectrum Disorder Students with Their Academic and Social Transition at Community Colleges (Doctoral dissertation). Retrieved from https://fisherpub.sjfc.edu/cgi/viewcontent.cgi ?referer=https: //www.google.com/\&httpsredir=1\&article=1220\&context=education_etd

Swanson, J. L. (2008). An analysis of the impact of high school dual enrollment course participation on post-secondary academic success, persistence and degree completion (Doctoral thesis). Retrieved from ProQuest Dissertations and Theses database (No. 3323472)

Symes, W., \& Humphrey, N. (2012). Including pupils with autistic spectrum disorders in the classroom: The role of teaching assistants. European Journal of Special Needs Education, 27(4), 517-532. doi:10.1080/08856257.2012.726019

Szidon, K., Ruppar, A., \& Smith, L. (2015). Five steps for developing effective transition plans for high school students with autism spectrum disorder. TEACHING Exceptional Children, 47(3), 147-152. doi:10.1177/0040059914559780

Terzian, M., Moore, K. A., Hamilton, K., Wallace Foundation, \& Child Trends. (2009). Effective and Promising Summer Learning Programs and Approaches for Economically-Disadvantaged Children and Youth: A White Paper for the Wallace Foundation. Wallace Foundation. Retrieved from http://libproxy.chapman.edu /login?url=http://search.ebscohost.com/login.aspx?direct=true\&AuthType=ip,uid $\& \mathrm{db}=$ eric $\& A N=E D 506969 \&$ site $=$ eds-live

Tinto, V. (1975). Dropout from higher education: A theoretical synthesis of recent research. Review of Educational Research, 45(1), 89-125. doi:10.3102/00346543045001089 
Tinto, V. (1993) Leaving college: Rethinking the causes and cures of student attrition, 2nd(ed.), Chicago: University of Chicago Press.

Ulug, M., Ozden, M. S., \& Eryilmaz, A. (2011). The Effects of Teachers' Attitudes on Students' Personality and Performance. Procedia - Social and Behavioral Sciences, 30, 738-742. doi:10.1016/j.sbspro.2011.10.144

University of Southern California. (n.d.). California Community Colleges. In Center for Urban Education. Retrieved from https://cue.usc.edu/research/californiacommunity-colleges/

Upadyaya, K., \& Salmela-Aro, K. (2013). Development of school engagement in association with academic success and well-being in varying social contexts: A review of empirical research. European Psychologist, 18(2), 136-147. doi:110.1027/1016-9040/a000143

Vanbergeijk, E., Klin, A., \& Volkmar, F. (2008). Supporting more able students on the autism spectrum: College and beyond. Journal of Autism and Developmental Disorders, 38(7), 1359-1370. doi:10.1007/s10803-007-0524-8

Vogel, S. A., Leyser, Y., Burgstahler, S., Sligar, S. R., \& Zecker, S. G. (2006). Faculty Knowledge and Practices Regarding Students with Disabilities in Three Contrasting Institutions of Higher Education. Journal of Postsecondary Education \& Disability, 18(2), 109. Retrieved from http://libproxy.chapman.edu/login ?url=http://search.ebscohost.com/login.aspx?direct=true \&AuthType=ip,uid\&db=e do\&AN=20222584\&site $=$ eds-live 
Wagner, M., Newman, L., Cameto, R., Levine, P., Marder, C., \& Institute of Education Sciences (ED), W. D. (2007). Perceptions and Expectations of Youth with Disabilities. A Special Topic Report of Findings from the National Longitudinal Transition Study-2 (NLTS2). NCSER 2007-3006. National Center for Special Education Research. Retrieved from http://libproxy.chapman.edu/login?url $=$ http://search.ebscohost.com/login.aspx?direct=true\&AuthType=ip,uid \&db=eric $\& \mathrm{AN}=\mathrm{ED} 498185 \&$ site $=$ eds-live

Wallace, L. C. (2016). Students with autism spectrum disorder in postsecondary education (Doctoral dissertation). Retrieved from ProQuest Dissertations \& Theses database. (No. 10158541)

Wei, X., Yu, J., Shattuck, P., McCracken, M., \& Blackorby, J. (2012). Science, Technology, Engineering, and Mathematics (STEM) Participation Among College Students with an Autism Spectrum Disorder. Journal of Autism \& Developmental Disorders, 43(7), 1539-1546. doi:10.1007/s10803-012-1700-Z

Wei, X., Christiano, E., Yu, J., Blackorby, J., Shattuck, P., \& Newman, L. (2014). Postsecondary Pathways and Persistence for STEM Versus Non-STEM Majors: Among College Students with an Autism Spectrum Disorder...science, technology, engineering, mathematics. Journal of Autism \& Developmental Disorders, 44(5), 1159-1167. doi:10.1007/s10803-013-1978-5

Wei, X., Wagner, M., Hudson, L., Yu, J. W., \& Javitz, H. (2016). The effect of transition planning participation and goal-setting on college enrollment among youth with autism spectrum disorders. Remedial and Special Education, 37(1), 3-14. doi:10.1177/0741932515581495 
Welkowitz, L. A., \& Baker, L. J. (2005). Supporting college students with Asperger's syndrome. In L. J. Baker \& L. A. Welkowitz (Eds.), Asperger's syndrome: Intervening in schools, clinics and communities (pp. 173-187). Mahwah, NJ: Erlbaum.

White, S., Ollendick, T., \& Bray, B. (2011). College students on the autism spectrum: Prevalence and associated problems. Autism, 15(6), 683-701. doi:10.1177/1362361310393363

White, S. W., Elias, R., Capriola-Hall, N. N., Smith, I. C., Conner, C. M., Asselin, S. B., \& Howlin, P. (2017). Development of a college transition and support program for students with autism spectrum disorder. Journal of Autism and Developmental Disorders, 47(10), 3072-3078. doi:10.1007/s10803-017-3236-8

Williams, D. (2014, January 21). Models of Disability. In Polly's pages (aka 'Donna Williams'). Retrieved from https://blog.donnawilliams.net/2014/01/21/models-ofdisability/

Wilson, K., Getzel, E., \& Brown, T. (2000). Enhancing the post-secondary campus climate for students with disabilities. Journal of Vocational Rehabilitation, 14(1), 37-50. Retrieved from https://thinkcollege.net/sites/default/files/files /resources/Wilson_enhancing.pdf

Wing, L. (1981). Asperger's Syndrome: A clinical account. Psychological Medicine, 11(1), 115-129. doi:10.1017/S0033291700053332. 
Wing, L., \& Potter, D. (2002). The Epidemiology of Autistic Spectrum Disorders: Is the Prevalence Rising? Mental Retardation and Developmental Disabilities Research Reviews, (8). 151-161. Retrieved from http://eds.b.ebscohost.com.libproxy .chapman.edu/eds/pdfviewer/pdfviewer?vid=9\&sid=3a39373a-6868-4ccb-bb0107f7ee559852\%40pdc-v-sessmgr03

Wolf, L. E., Brown, J. T., \& Bork, G. R. (2009). Students with Asperger Syndrome: A guide for college personnel. Shawnee Mission, KN: Autism Asperger.

Zager, D., \& Alpern, C. (2010). College-based inclusion programming for transition-age students with autism. Hammill Institute on Disabilities, 25(3), 151-157. doi:10.1177/1088357610371331

Zahavi, D., and J. Parnas. (2003). Conceptual problems in infantile autism research: Why cognitive science needs phenomenology. Journal of Consciousness Studies. 10(9/10), 53-71. Retrieved from https://www.researchgate.net/profile /Dan_Zahavi/publication/233614951_Conceptual_Problems_in_Infantile_Autism _Research_Why_Cognitive_Science_Needs_Phenomenology/links/569c9d5c08a eeea985a64b39/Conceptual-Problems-in-Infantile-Autism-Research-WhyCognitive-Science-Needs-Phenomenology.pdf 